\title{
Branes and quantization
}

\author{
Sergei Gukov ${ }^{1,2}$ and Edward Witten ${ }^{3}$ \\ ${ }^{1}$ Department of Physics, University of California, Santa Barbara, \\ CA 93106, USA \\ ${ }^{2}$ Department of Physics, Caltech, Pasadena, CA 91125, USA \\ ${ }^{3}$ School of Natural Sciences, Institute for Advanced Study, Princeton, \\ NJ 08540, USA \\ gukov@theory . caltech.edu
}

\begin{abstract}
The problem of quantizing a symplectic manifold $(M, \omega)$ can be formulated in terms of the $A$-model of a complexification of $M$. This leads to an interesting new perspective on quantization. From this point of view, the Hilbert space obtained by quantization of $(M, \omega)$ is the space of $\left(\mathcal{B}_{\mathrm{cc}}, \mathcal{B}^{\prime}\right)$ strings, where $\mathcal{B}_{\mathrm{cc}}$ and $\mathcal{B}^{\prime}$ are two $A$-branes; $\mathcal{B}^{\prime}$ is an ordinary Lagrangian $A$-brane, and $\mathcal{B}_{\text {cc }}$ is a space-filling coisotropic $A$-brane. $\mathcal{B}^{\prime}$ is supported on $M$, and the choice of $\omega$ is encoded in the choice of $\mathcal{B}_{\mathrm{cc}}$. As an example, we describe from this point of view the representations of the group $S L(2, \mathbb{R})$. Another application is to Chern-Simons gauge theory.
\end{abstract}

\section{CONTEnTs}


1.4 Comparison to deformation quantization

1455

1.5 The inverse problem

1456

1.6 Organization of this paper

1458

2 Basic construction

2.1 The $A$-model and the canonical coisotropic brane

2.2 Space of $\left(\mathcal{B}_{\mathrm{cc}}, \mathcal{B}_{\mathrm{cc}}\right)$ Strings

1461

2.3 Lagrangian brane and quantization

1462

2.4 Unitarity 1470

3 Branes and representations 1473

3.1 An example 1473

$3.2 \quad$ Representations of $S U(2)$ 1479

3.3 Discrete series of $S L(2, \mathbb{R})$ 1481

3.4 Principal series of $S L(2, \mathbb{R})$ 1482

3.5 Algebraic description 1485

3.6 Discrete series of $\widetilde{S L}(2, \mathbb{R})$ 1490

3.7 Harish-Chandra modules from branes 1491

$3.8 \tilde{\tau}$-Invariant branes with only asymptotic $S L(2, \mathbb{R})$ symmetry

$3.9 \quad$ Relation to $\mathcal{D}$-modules 1504

3.10 Groups of higher rank 1509

4 Quantization of Chern-Simons gauge theory 1510

Acknowledgments 1516

References

1516 


\section{Introduction}

\subsection{The Problem}

According to textbooks, the passage from classical mechanics to quantum mechanics is made by replacing Poisson brackets with commutators. However, this is an unrealistically simple description of the situation, even for a basic example such as the classical phase space $\mathbb{R}^{2}$, with canonically conjugate variables $x$ and $p$. One can associate a quantum operator $\mathcal{O}_{f}$ to a classical function $f(x, p)$, but not in a completely unique way, because of what textbooks call the operator ordering problem. Regardless of how one defines $\mathcal{O}_{f}$, the map from classical functions $f$ to quantum operators $\mathcal{O}_{f}$ does not map Poisson brackets to commutators. Only if one restricts oneself to functions that are at most quadratic in $x$ and $p$ does one have the simple relation

$$
\left[\mathcal{O}_{f}, \mathcal{O}_{g}\right]=-i \hbar \mathcal{O}_{\{f, g\}}
$$

The notion of a function being at most quadratic in $x$ and $p$ is not invariant under canonical transformations. Quantizations of $\mathbb{R}^{2}$ with different choices of what one means by linear or quadratic functions are not the same. One cannot conjugate one such quantization to another by a unitary map between the two Hilbert spaces that transforms the operators $\mathcal{O}_{f}$ constructed in one quantization to their counterparts $\tilde{\mathcal{O}}_{f}$ in another quantization. The order $\hbar^{2}$ corrections to (1.1) are simply different in the two quantizations.

The fact that quantization is ambiguous locally also means that it is not clear how to carry out quantization globally. Suppose that $M$ is a $2 n$-dimensional classical phase space that we wish to quantize. (And suppose that we are given on $M$ an additional structure known as a prequantum line bundle [1,2]; this will enter our story shortly.) Even if one can locally identify $M$ with $\mathbb{R}^{2 n}$, this does not automatically tell us how to quantize $M$, even locally, since the quantization of $\mathbb{R}^{2 n}$ is not unique, as we have just explained. If we make random local choices in quantizing $M$, we cannot expect them to fit together to a sensible global quantization. There is also no good framework for trying to fit the pieces together, because there is no general notion of restricting a quantization of $M$ to a quantization of an open subset of $M$, which would be a prerequisite for trying to quantize $M$ by gluing together quantizations of open subsets. 
One cannot expect to be able to quantize $M$ without some additional structure beyond its classical symplectic structure (and prequantum line bundle). There is no known general recipe for what this additional structure should be. As a result, there is no general theory of quantization of classical phase spaces.

In practice, quantization is a somewhat informal notion, which refers to a collection of loosely related procedures. The most important example in which we know what quantization should mean is $\mathbb{R}^{2 n}$ with a given choice of affine structure, that is, a choice of what one means by linear functions. This can be quantized in a way that requires no further choices. (In the usual procedure, one splits the linear functions into coordinates and momenta, which are then taken to act by multiplication and differentiation, respectively. The resulting Hilbert space admits a natural action of the symplectic group $S p(2 n, \mathbb{R})$ or rather its double cover, and thus does not really depend on the splitting between coordinates and momenta.) Another important example is a cotangent bundle $M=T^{*} U$ (with the standard symplectic structure), which can be quantized in a natural way in terms of half-densities on $U$; similarly, there is a natural procedure for quantization of Kahler manifolds by taking holomorphic sections of the appropriate line bundle. Finally, if one knows how to quantize $M$, and $G$ is a group that acts on $M$, then (under some mild restrictions) one can define a quantization of the symplectic quotient $M / / G$ by taking the $G$-invariant part of the quantization of $M$. There are various ways to combine the procedures just mentioned.

There is no guarantee that the different procedures are equivalent. If $M$ is a cotangent bundle or a Kahler manifold in more than one way or a symplectic quotient of one of these in more than one way, or can be realized by more than one of these constructions, there is no assurance that the different procedures lead to equivalent quantizations.

\subsection{Quantization via branes}

In this paper, we offer a new perspective on quantization, based on twodimensional sigma models. The goal is to get closer to a systematic theory of quantization. However, it is not clear to what extent our perspective helps in computing new formulas.

Our procedure is as follows. We start with a symplectic manifold $M$, with symplectic form $\omega$, that we wish to quantize. As in geometric quantization $[1,2]$, we assume that $M$ is endowed with a prequantum line bundle $\mathcal{L}$; this is a complex line bundle $\mathcal{L} \rightarrow M$ with a unitary connection of curvature $\omega$. 
For our purposes, saying that $Y$ is a complexification of $M$ simply means that (1) $Y$ is a complex manifold with an antiholomorphic involution ${ }^{1}$ $\tau: Y \rightarrow Y$, such that $M$ is a component of the fixed point set of $\tau ;$ (2) the symplectic form $\omega$ of $M$ is the restriction to $M$ of a nondegenerate holomorphic two-form $\Omega$ on $Y$, such that $\tau^{*} \Omega=\bar{\Omega}$; (3) the unitary line bundle $\mathcal{L} \rightarrow M$ can be extended to a unitary line bundle $\mathcal{L} \rightarrow Y$ with a connection of curvature $\operatorname{Re} \Omega$, and moreover the action of $\tau$ on $Y$ lifts to an action on $\mathcal{L}$, restricting to the identity on $M$. These data are regarded as part of the definition of $Y$.

The case of most interest in the present paper is the case that $Y$ is an affine variety, which roughly means that it admits plenty of holomorphic functions. More precisely, an affine variety is defined by a finite set of polynomial equations for a finite set of complex variables $x_{1}, \ldots, x_{s}$, as opposed to a more general algebraic variety which is obtained by gluing together pieces that are each affine varieties. Our approach to quantization will be based on the $A$-model associated with the real symplectic form $\omega_{Y}=\operatorname{Im} \Omega$. So we need a further condition on $Y$, which ensures that this theory has a good $A$-model. A good $A$-model is one in which the relevant correlation functions and other observables are complex-valued, rather than being functions of a formal deformation parameter. (For example, the most familiar $A$-model observables are obtained from sums over worldsheet instantons of different degrees. Having a good $A$-model means that such sums are not just formal power series but converge to complex-valued functions.) $Y$ will have a good $A$-model if the supersymmetric sigma-model with target $Y$, which can be twisted to give the $A$-model, is well-behaved quantum mechanically; this in turn should be true if $Y$ admits a complete hyper-Kahler metric, compatible with its complex symplectic structure. For instance, the example considered below and in more detail in Section 3 corresponds to the EguchiHansen manifold, which is a complete hyper-Kahler manifold. Having a good $A$-model should imply that deformation quantization of $Y$ (which is part of the $A$-model, as we discuss below) gives an actual deformation of the ring of holomorphic functions on $Y$, with a complex deformation parameter; a bad $A$-model merely leads to a formal deformation over a ring of formal power series.

We require an actual deformation of the ring of functions, not just a formal one, for our approach to quantization to make sense. Interestingly, the conditions [3] under which deformation quantization of an affine variety gives an actual deformation of the ring of functions on $Y$ are very similar to the conditions for $Y$ to admit a complete hyper-Kahler metric along the lines of the complete Calabi-Yau metrics constructed in [4].

\footnotetext{
${ }^{1}$ An involution is simply a symmetry whose square is the identity.
} 
The most familiar $A$-branes are Lagrangian $A$-branes, supported on a Lagrangian submanifold of $Y$; such a submanifold is of middle dimension. In general [5], however, the $A$-model can also admit $A$-branes whose support has a dimension greater than one-half the dimension of $Y$. The support of such a brane is a coisotropic submanifold of $Y$ with certain somewhat special properties. In particular, the choice of the line bundle $\mathcal{L} \rightarrow M$ with curvature $\operatorname{Re} \Omega$ determines, in the language of [6], a canonical coisotropic brane in the $A$-model of $Y$. Its support is all of $Y$ and it will be one of the main ingredients in the present paper.

Suggestions that the $A$-model is related to deformation quantization (whose relation to quantization is discussed in Section 1.4) go back to [7] and [8] and have been extended and made more precise in $[9,10]$, partly in the framework of generalized complex geometry [11,12]. The canonical coisotropic $A$-brane was used in [6] to elucidate some of these matters. For a related approach to the $A$-model, see [13].

We will also make use of ordinary Lagrangian $A$-branes. $M$ itself is a Lagrangian submanifold, so (if $M$ obeys a mild topological condition) we can define a rank $1 A$-brane supported on $M$. Let us pick such a brane (there are inequivalent choices if $M$ is not simply connected) and call it $\mathcal{B}^{\prime}$. In this paper, we write $\mathcal{B}_{\text {cc }}$ for the canonical coisotropic $A$-brane, $\mathcal{B}^{\prime}$ for a Lagrangian $A$-brane, and $\mathcal{B}$ for an $A$-brane of unspecified type.

Quantization of $M$ is now achieved by declaring that the Hilbert space associated to $M$ is the space $\mathcal{H}$ of $\left(\mathcal{B}_{\mathrm{cc}}, \mathcal{B}^{\prime}\right)$ strings. This definition certainly gives a vector space associated to the choice of $A$-brane $\mathcal{B}^{\prime}$. That the explicit construction of this vector space is similar to quantization was originally shown by Aldi and Zaslow in examples [14], and will be further discussed in Section 2.

To justify calling this process quantization, we need more structure. For one thing, we want to associate to $\mathcal{B}^{\prime}$ not just a vector space but a Hilbert space. It is unusual to get a Hilbert space structure in the topological $A$-model, but in the present context, as explained in Section 2.4, $\mathcal{H}$ can be given a hermitian metric by making use of the antiholomorphic involution $\tau$. The space $\mathcal{H}$, with its hermitian metric, depends only on the choices of $Y, \mathcal{L}$, and $\mathcal{B}^{\prime}$, and not on any additional data (such as a metric on $Y$ ) that is used in defining the $A$-model. We do not have a general proof that the hermitian metric on $\mathcal{H}$ is positive definite, though this is true near the classical limit. (A generalization of the construction, involving an antiholomorphic involution that maps $M$ to itself but does not leave $M$ fixed pointwise, leads to a hermitian metric on $\mathcal{H}$ that is not positive-definite near the classical limit.) 
If our procedure is reasonably to be called quantization, we also want to have a natural way to quantize a large class of functions on $M$, that is to realize them as operators on $\mathcal{H}$. The functions on $M$ that can be naturally quantized, in our approach, are the functions that are restrictions of holomorphic functions on $Y$ that have a suitable behavior at infinity. The details of what is suitable behavior at infinity are tied to the question of what spaces have good $A$-models. However, for $Y$ an affine variety (defined by a finite set of polynomial equations for a finite set of complex variables $\left.x_{1}, \ldots, x_{s}\right)$, a reasonable condition is to allow only functions of polynomial growth (that is, polynomials in the $x_{i}$ ). This gives a very large class of holomorphic functions on such a variety, and it is for this class of functions that (under certain restrictions on $Y$ ) deformation quantization gives an actual rather than formal deformation. So this is the right class of functions to consider. For reasonable $M \subset Y$, the restrictions of these functions are dense in the space of smooth functions on $M$, and our procedure leads to quantization in the sense of constructing a Hilbert space $\mathcal{H}$ with a map from a large class of functions on $M$ to operators on $\mathcal{H}$. At the opposite extreme, if $Y$ is compact, the definition of the Hilbert space $\mathcal{H}$ still makes sense, but we get no operators acting on this Hilbert space; one might not want to call this quantization.

To make this discussion a little more concrete, we will consider an example (which will be explored more fully in Section 3). Let $M=S^{2}$ be a twosphere, and let $\omega$ be a symplectic form on $M$ with $\int_{M} \omega=2 \pi n, n \in \mathbb{Z}$. We expect quantization to give a Hilbert space $\mathcal{H}$ of dimension $n$. The infinitedimensional group Adiff $S^{2}$ of area-preserving diffeomorphisms of $S^{2}$ acts on the classical phase space $(M, \omega)$. The group that acts on $\mathcal{H}$ is $U(n)$. There is no natural way to map Adiff $S^{2}$ to $U(n)$, so any approach to quantization will involve some arbitrary choices.

One standard approach in this problem (which in geometric quantization $[1,2]$ is known as picking a complex polarization) is to pick a complex structure $J$ on $S^{2}$, such that $\omega$ is of type $(1,1)$. The subgroup of Adiff $S^{2}$ that preserves $J$ is at most $S O(3)$, and it is convenient to pick $J$ so that this subgroup is actually $S O(3)$. If so, with some choice of coordinates, the Kahler metric on $S^{2}$ is a multiple of the round metric on the two-sphere $x^{2}+y^{2}+z^{2}=1$. Quantization is now straightforward: one defines $\mathcal{H}$ to be $H^{0}\left(S^{2}, \mathcal{L}\right)$, the space of holomorphic sections of $\mathcal{L}$ in complex structure $J$. Since the procedure of quantization was $S O(3)$-invariant, the group $S U(2)$ (the universal cover of $S O(3)$ ) acts naturally on $\mathcal{H}$, as does its Lie algebra. The functions $x, y$, and $z$ generate via Poisson brackets the action of this Lie algebra, so in this approach to quantization, these functions naturally map to quantum operators. One can then in a fairly natural way take polynomial functions in $x, y$, and $z$ to act on $\mathcal{H}$ by mapping a 
monomial $x^{a} y^{b} z^{c}$ to the corresponding symmetrized polynomial in the $\mathfrak{s o}(3)$ generators.

Clearly, we could have embedded $S O(3)$ in Adiff $S^{2}$ in many (conjugate) ways, so this approach to quantization depends on an arbitrary choice. Now let us discuss how one would quantize the same example in our approach. We are supposed to pick a suitable complexification $Y$ of $S^{2}$. We do this by again picking coordinates in which $S^{2}$ is defined by the equation

$$
x^{2}+y^{2}+z^{2}=1
$$

and we introduce $Y$ by simply regarding $x, y$, and $z$ as complex variables. Thus $Y$ is an affine variety; it admits a complete hyper-Kahler metric (the Eguchi-Hansen metric), so we expect it to have a good $A$-model (the relevant deformation of the ring of functions on $Y$ is explicitly described in Section 3.1, and involves a complex parameter, not a formal variable). The allowed holomorphic functions are polynomials in $x, y$, and $z$ subject to the relation (1.2). On $Y$, there is a holomorphic two-form $\Omega$ which restricts on $M$ to the properly normalized symplectic form $\omega$; it is simply $\Omega=n d x \wedge d y / 2 z$. The holomorphic functions on $Y$ with polynomial growth at infinity are simply the polynomials in $x, y$, and $z$, so the functions that we can quantize are those polynomials, just as in the previous and more standard approach. In the standard approach, the special role of $x, y$, and $z$ is that they generate via Poisson brackets symmetries of the complex structure that is used in quantization. In our approach, what is special about $x, y$, and $z$ is that they generate the ring of holomorphic functions on $Y$ with polynomial growth at infinity.

It is illuminating to consider an alternative complexification of $S^{2}$ that does not work well. We can define the two sphere by the equation ${ }^{2} \tilde{x}^{4}+\tilde{y}^{4}+$ $\tilde{z}^{4}=1$, for real variables $\tilde{x}, \tilde{y}, \tilde{z}$, so one can define a complexification $\tilde{Y}$ of $S^{2}$ by letting $\tilde{x}, \tilde{y}$, and $\tilde{z}$ be complex variables obeying the same equation. But deformation quantization of $\tilde{Y}$ is only a formal procedure according to [3], and the construction of [4] does not endow $\tilde{Y}$ with a complete Calabi-Yau metric. Rather, $\tilde{Y}$ admits an incomplete Calabi-Yau metric, which can be compactified to give a K3 surface. We expect that to give $\tilde{Y}$ a good $A$-model, one must compactify it and consider the $A$-model of the K3-surface; then there are no holomorphic functions and no natural interpretation in terms of quantization.

\footnotetext{
${ }^{2}$ To show that this equation defines a two sphere, observe that if $\tilde{x}, \tilde{y}$, and $\tilde{z}$ are real numbers obeying $\tilde{x}^{4}+\tilde{y}^{4}+\tilde{z}^{4}=1$, there is a unique positive $t$ such that $(x, y, z)=t(\tilde{x}, \tilde{y}, \tilde{z})$ obey $x^{2}+y^{2}+z^{2}=1$. This map gives an isomorphism between the space of solutions of $x^{2}+y^{2}+z^{2}=1$ and the space of solutions of $\tilde{x}^{4}+\tilde{y}^{4}+\tilde{z}^{4}=1$.
} 


\subsection{Comparison to geometric quantization}

Here and in Section 1.4, we will compare our approach to some standard approaches to quantization.

In geometric quantization $[1,2]$, the first step, given $(M, \omega)$, is to pick a prequantum line bundle $\mathcal{L} \rightarrow M$, that is, a unitary line bundle with a connection of curvature $\omega$. This is also an initial step in our approach, as explained above, and probably (explicitly or implicitly) in any approach to quantization. The second step is then to pick a polarization (typical examples being a realization of $M$ as a cotangent bundle $T^{*} U$ for some $U$, or a choice of Kahler structure on $M)$, after which quantization is carried out via half-densities on $U$ or holomorphic sections of $\mathcal{L} \otimes K^{1 / 2} \rightarrow M\left(K^{1 / 2}\right.$ is a square root of the canonical bundle of $M$ ).

This second step has some drawbacks. A global polarization may not exist, even for phase spaces that should be quantizable. Moreover, if a polarization exists, there are many possible polarizations. It is not clear when quantization carried out with two different polarizations gives equivalent results.

Our approach has analogous drawbacks. Given $(M, \omega)$, it is not clear whether a suitable $Y$ exists, or whether different choices of $Y$ will give equivalent results. (We do not know of any examples in which this is the case.) Our approach is therefore particularly useful if there is a natural $Y$ (or at least a natural class of $Y$ 's with some special relationship), while geometric quantization is particularly useful if there is a natural polarization.

The problem of when geometric quantization with two different polarizations gives equivalent results is vexing. The most important example is quantization of $\mathbb{R}^{2 n}$. Once one picks an affine structure on $\mathbb{R}^{2 n}$ (a notion of what one means by linear functions), a polarization can be picked by choosing a maximal Poisson-commuting set of linear functions $q^{1}, \ldots, q^{n}$, which we declare to be the coordinates (as opposed to the momenta). Quantization is then carried out by introducing a Hilbert space of functions (actually half-densities) $\Psi\left(q^{1}, \ldots, q^{n}\right)$. We may call a choice of this kind a linear polarization. It is a classic result that quantizations with different linear polarizations (compatible with the same affine structure) are equivalent. The usual proof uses the action of the symplectic group $S p(2 n, \mathbb{R}$ ) (or rather its double cover), generated by quadratic functions of the coordinates and momenta.

This fundamental example has others as corollaries. For $G$ a subgroup of the symplectic group, consider the quantization of the symplectic quotient 
$M=\mathbb{R}^{2 n} / / G$. Any $G$-invariant polarization of $\mathbb{R}^{2 n}$ descends to a polarization of $M$, and geometric quantization of $M$ with two polarizations that descend from $G$-invariant linear polarizations of $\mathbb{R}^{2 n}$ will be equivalent. This statement, which follows from the equivalence of linear polarizations of $\mathbb{R}^{2 n}$, also has an analog for $n=\infty$ in the case of Chern-Simons gauge theory [15].

An example of a well-motivated procedure of quantization that is awkward to describe in geometric quantization is the case that $M=\mathbb{R}^{2 n} / / G$ is the symplectic quotient of $\mathbb{R}^{2 n}$ (or some other space that can be quantized by geometric quantization) by a subgroup $G \subset S p(2 n, \mathbb{R})$ such that there is no $G$-invariant polarization of $\mathbb{R}^{2 n}$. It is natural to define quantization of $M$ by taking the $G$-invariant part of the quantization of $\mathbb{R}^{2 n}$, but this definition is not related in any obvious way to what one can get from a polarization of $M$.

In our approach to quantization via branes, near the semiclassical limit, one may define the $A$-model of $Y$ by picking a suitable metric on $Y$. This is the analog of a polarization in our approach. One illuminating and important case is that the metric on $Y$ is a complete hyper-Kahler metric and $M$ is a complex submanifold in one of the complex structures, which we will call $J$. We will say that a metric of this kind gives a hyper-Kahler polarization of the pair $(Y, M)$. In this case, $J$ defines a complex polarization of $M$ in the sense of geometric quantization, and the vector space $\mathcal{H}$ that is defined in our procedure (but not in general its hermitian inner product) agrees with what one would naturally define in geometric quantization, as we explain in Section 2.3.

The main advantage of our approach may be that the question of what can be varied without changing the quantization is perhaps clearer than it is in geometric quantization. As we have stressed, our answer to the question "Upon what additional data does a quantization of $(M, \omega)$ depends?" is "It depends on the choice of the complexification $Y$ with antiholomorphic involution $\tau$, holomorphic two-form $\Omega$ and line bundle $\mathcal{L}$."

The fact that different linear polarizations of $\mathbb{R}^{2 n}$ lead to equivalent quantizations is a special case of our statement. Given $\mathbb{R}^{2 n}$ with real-valued linear coordinates $x^{1}, \ldots, x^{2 n}$ and symplectic structure $\omega=\sum_{i<j} \omega_{i j} d x^{i} \wedge d x^{j}$, we define $Y, \Omega$ without breaking the $S p(2 n, \mathbb{R})$ symmetry by complexifying the $x^{i}$ and setting $\Omega=\sum_{i<j} \omega_{i j} d x^{i} \wedge d x^{j}$. We define $\tau$ to act by $x^{i} \rightarrow \bar{x}^{i}$. Since $Y$ is contractible, $\mathcal{L}$ exists and is unique up to isomorphism, so in our approach, quantization of $\mathbb{R}^{2 n}$ endowed with an affine structure is natural. The group $\operatorname{Sp}(2 n, \mathbb{R})$ of symmetries of the structure (and in fact, its inhomogeneous extension to include additive shifts of the coordinates) must therefore act, at least projectively. 


\subsection{Comparison to deformation quantization}

Deformation quantization [16] is another matter. Unlike quantization, deformation quantization is a systematic procedure. Starting with a symplectic manifold $M$ - or more generally, any Poisson manifold - deformation quantization produces a deformation of the ring of functions on $M$, depending on a formal parameter $\hbar$. This can be done in a way that, up to a natural automorphism, does not depend on any auxiliary choice (such as the choices that are needed in quantization). The theory of deformation quantization has led to beautiful results [17] that can be expressed in terms of two-dimensional quantum field theory [18], somewhat like our approach to quantization.

Since deformation quantization is a formal procedure, it makes sense for complex manifolds. In other words, if $Y$ is a complex symplectic manifold (such as an affine variety) that admits many holomorphic functions, one can apply deformation quantization to deform the ring of holomorphic functions on $Y$ to an associative but noncommutative algebra [3]. Deformation quantization of the ring of holomorphic functions on $Y$ requires no arbitrary choices (beyond the structure of $Y$ as a complex symplectic manifold) but quantization does.

However, deformation quantization is not quantization. Generically, it leads to a deformation over a ring of formal power series (in the formal variable $\hbar$ ), not a deformation with a complex parameter. It does not lead to a natural Hilbert space $\mathcal{H}$ on which the deformed algebra acts. In our earlier example of quantizing a two-sphere whose area is $2 \pi n$, quantum mechanics requires that $n$ (which becomes the dimension of $\mathcal{H}$ ) should be an integer, while in deformation quantization, $\hbar=1 / n$ is treated as a formal variable and there is no special behavior when $\hbar^{-1}$ is an integer.

Generally speaking, physics is based on quantization, rather than deformation quantization, although conventional quantization sometimes leads to problems that can be treated by deformation quantization. For a well-known example, see [19]. Our approach to quantization does have a relationship to deformation quantization. The relation is that deformation quantization of $Y$ produces an algebra that then acts in the quantization of a real symplectic submanifold $M \subset Y$. (See [20-22] for a similar perspective in the context of representation theory.) This will be explained in the next subsection.

As already noted, in our framework, the existence of a good $A$-model for $Y$ is supposed to ensure that deformation quantization of $Y$ produces an actual deformation of the algebra of holomorphic functions, depending on a 
complex parameter $\hbar$ (or $1 / n$ ), not just a formal deformation depending on a formal parameter $\hbar$.

\subsection{The inverse problem}

In describing our approach to quantization, we followed tradition and began with a symplectic manifold $(M, \omega)$ that one wishes to quantize. The solution to the problem involves picking a suitable complexification $(Y, \Omega)$.

There is an alternative approach in which the starting point is a complex symplectic manifold $(Y, \Omega)$, together with a unitary line bundle $\mathcal{L} \rightarrow Y$ of curvature $\operatorname{Re} \Omega$. The following discussion is most interesting if $Y$ has plenty of holomorphic functions. This is so if $Y$ is an affine variety, such as the variety $x^{2}+y^{2}+z^{2}=1$ that featured in the example that we discussed previously.

Then one considers the $A$-model of $Y$ in symplectic structure $\omega_{Y}=\operatorname{Im} \Omega$. The choice of $\mathcal{L}$ enables us, following [5] and [6], to define a coisotropic $A$-brane $\mathcal{B}_{\text {cc }}$, whose support is all of $Y$. For any $A$-brane $\mathcal{B}$, the space of $(\mathcal{B}, \mathcal{B})$ strings is a $\mathbb{Z}$-graded associative algebra. In the present case, additively, the space of $\left(\mathcal{B}_{\mathrm{cc}}, \mathcal{B}_{\mathrm{cc}}\right)$ strings is just the space of holomorphic functions on the complex manifold $Y$. However, in the $A$-model, the commutative ring of holomorphic functions is deformed. The first-order deformation is by the Poisson bracket, and the higher order corrections (which can be computed in sigma-model perturbation theory, somewhat as in [18]) are controlled by associativity. Thus the space of $\left(\mathcal{B}_{\mathrm{cc}}, \mathcal{B}_{\mathrm{cc}}\right)$ strings is an associative but noncommutative algebra $\mathcal{A}$ that we can think of as arising from deformation quantization of $Y$. As we have stressed, if $Y$ has a good $A$-model, this deformation involves an actual complex parameter, not a formal one. Moreover, if $Y$ admits a good $A$-model, its symmetries that preserve a coisotropic $A$-brane $\mathcal{B}_{\mathrm{cc}}$ will act on $\mathcal{A}$ as automorphisms. In our approach, these are precisely the symplectomorphisms of the complex symplectic manifold $(Y, \Omega)$. This is closely related to what one finds in deformation quantization of $Y$ when it produces an actual deformation of the algebra of holomorphic functions, not just a formal deformation (see [23] for a detailed discussion of the affine space).

If we are interested in quantization, as opposed to deformation quantization, we need something smaller that $\mathcal{A}$ acts on. For this, we note first that if $\mathcal{B}$ is any other $A$-brane, then by general principles $\mathcal{A}$ acts on the space of $\left(\mathcal{B}_{\mathrm{cc}}, \mathcal{B}\right)$ strings. Now, pick $\mathcal{B}$ to be a conventional Lagrangian $A$-brane, supported on a Lagrangian submanifold $M \subset Y$. We denote this brane as $\mathcal{B}^{\prime}$. Then the space $\mathcal{H}$ of $\left(\mathcal{B}_{\mathrm{cc}}, \mathcal{B}^{\prime}\right)$ strings admits a natural action of the algebra $\mathcal{A}$. 
Now suppose further that $M$ has been chosen so that $\operatorname{Re} \Omega$ remains nondegenerate when restricted to $M$. Analysis of the $\left(\mathcal{B}_{\mathrm{cc}}, \mathcal{B}^{\prime}\right)$ strings, as first considered in examples in [14], relates $\mathcal{H}$ to quantization of $M$ with symplectic structure $\operatorname{Re} \Omega$. But how can we get in this framework a hermitian inner product - usually understood as one of the main points of quantization? For this, we need one more piece of data: an antiholomorphic involution $\tau: Y \rightarrow Y$ that obeys $\tau^{*} \Omega=\bar{\Omega}$, maps $M$ to itself, and lifts to an action on $\mathcal{L}$. With the help of $\tau$ (and more standard ingredients, such as CPT symmetry), one can define a hermitian inner product on $\mathcal{H}$, with the property that holomorphic functions on $Y$ that obey $\tau(\bar{f})=f$ act on $\mathcal{H}$ as hermitian operators. Near the classical limit, the hermitian inner product is positive definite if and only if $\tau$ leaves $M$ fixed pointwise.

A noteworthy point here is that the algebra $\mathcal{A}$ only depends on the input data $Y, \Omega, \mathcal{L}$, and not on $\tau$. If $\tau$ does not exist (or $M$ is not a component of the fixed point set of any $\tau$ ), then everything that we have said goes through, except that $\mathcal{H}$ is not endowed with a natural hermitian metric.

Alternatively, $Y$ may admit several different antiholomorphic involutions, say $\tau$ and $\tau^{\prime}$. Let $M$ and $M^{\prime}$ be components of the fixed points sets of $\tau$ and $\tau^{\prime}$ (and suppose that $\operatorname{Re} \Omega$ is nondegenerate when restricted to either one). Then we can quantize either $M$ or $M^{\prime}$ by the above procedure, giving Hilbert spaces $\mathcal{H}$ and $\mathcal{H}^{\prime}$. The same algebra $\mathcal{A}$ will act in either case. Functions that are real when restricted to $M$ will be hermitian as operators on $\mathcal{H}$, and functions that are real when restricted to $M^{\prime}$ will be hermitian as operators on $\mathcal{H}^{\prime}$.

There are far more choices if we are not interested in a hermitian metric. Then $M$ can be the support of any rank $1 A$-brane, and the same algebra $\mathcal{A}$ acts on the space $\mathcal{H}$ that we obtain by quantizing $M$, regardless of what $M$ we pick.

\section{The A-model}

So far we have emphasized two points of view about this subject.

In the first approach, the starting point is the real symplectic manifold $(M, \omega)$ that we wish to quantize. The problem is solved by complexifying $M$ to a complex symplectic manifold $(Y, \Omega)$ that has a good $A$-model for symplectic structure $\omega_{Y}=\operatorname{Im} \Omega$.

In the second approach, the starting point is the complex symplectic manifold $(Y, \Omega)$. Picking a suitable coisotropic brane $\mathcal{B}_{\mathrm{cc}}$, assumed to be an $A$-brane with respect to $\omega_{Y}=\operatorname{Im} \Omega$, we deform the algebra of holomorphic 
functions on $Y$ to a noncommutative algebra $\mathcal{A}$. Then picking another $A$-brane, we get a module for $\mathcal{A}$.

A third approach, and the most natural one from the point of view of twodimensional topological quantum field theory, is to emphasize the $A$-model of $Y$, regarded as a real symplectic manifold with symplectic structure $\omega_{Y}$. There may be many inequivalent choices of space-filling coisotropic $A$-brane $\mathcal{B}_{\text {cc }}$ on $Y$ - corresponding to different choices of a complex structure $I$ on $Y$ for which there is a holomorphic two-form $\Omega$ with $\omega_{Y}=\operatorname{Im} \Omega$. For each choice of such a brane $\mathcal{B}_{\text {cc }}$, we get a noncommutative algebra $\mathcal{A}$ that acts on the space of $\left(\mathcal{B}_{\mathrm{cc}}, \mathcal{B}^{\prime}\right)$ strings, for any other $A$-brane $\mathcal{B}^{\prime}$. If $M$ is the support of $\mathcal{B}^{\prime}$, the space of $\left(\mathcal{B}_{\mathrm{cc}}, \mathcal{B}^{\prime}\right)$ strings gives a quantization of $M$ whenever $\operatorname{Re} \Omega$ is nondegenerate when restricted to $M$. Thus, the same $A$-model can lead to quantization of $M$ in different symplectic structures.

\subsection{Organization of this paper}

In Section 2, we will describe in more detail our $A$-model approach to quantization.

Section 3 is devoted primarily to analyzing in more depth the example related to $M=S^{2}$. This example is surprisingly rich and related to representation theory of $S L(2, \mathbb{R})$ as well as $S U(2)$. In this paper, we consider primarily the case of those groups, but actually, as we explain at the end of Section 3, the example has a generalization in which $M$ is a coadjoint orbit of a semi-simple real Lie group of higher rank, and $Y$ is the corresponding orbit of its complexification. This leads to a perspective on the representations of semi-simple real Lie groups, similar to that of Brylinski [20-22].

Finally, in Section 4, we discuss from the present point of view one of the few known examples in which the subtleties of quantization are actually important for quantum field theory. This is three-dimensional Chern-Simons gauge theory.

\section{Basic construction}

\subsection{The $A$-model and the canonical coisotropic brane}

We begin with a complex symplectic manifold $Y$, that is, a complex manifold endowed with a nondegenerate holomorphic two-form $\Omega$. Though we will not assume that $Y$ has a hyper-Kahler structure, it is convenient to use a 
notation that is suggested by the hyper-Kahler case. We write $I$ for the complex structure of $Y$, and we denote the real and imaginary parts of $\Omega$ as $\omega_{J}$ and $\omega_{K}$ :

$$
\Omega=\omega_{J}+\mathrm{i} \omega_{K} .
$$

Since $\Omega$ is of type $(2,0)$, we have $\mathrm{I}^{\mathrm{t}} \Omega=i \Omega$, or

$$
\mathrm{I}^{\mathrm{t}} \omega_{J}=-\omega_{K}, \quad \mathrm{I}^{\mathrm{t}} \omega_{K}=\omega_{J} .
$$

(We regard $I$ as a linear transformation of tangent vectors; $\mathrm{I}^{\mathrm{t}}$ is the transpose map acting on one-forms. $\Omega$ and $\mathrm{I}^{\mathrm{t}} \Omega$ are maps from tangent vectors to one forms.)

In this paper, we view $Y$ as a real symplectic manifold with symplectic structure $\omega_{Y}=\omega_{K}$, and we study the associated $A$-model. The most familiar branes of the $A$-model are Lagrangian branes, supported on a Lagrangian submanifold that necessarily is of middle dimension. However [5], in general it is also possible to have an $A$-brane supported on a coisotropic submanifold $Z \subset Y$ whose dimension exceeds half the dimension of $Y$. For our purposes, we are interested in a rank 1 coisotropic $A$-brane whose support is simply $Z=Y$. Like any rank 1 brane, such a brane is endowed with a unitary line bundle $\mathcal{L}$ with a connection whose curvature we call $F$. The necessary condition for such a brane to be an $A$-brane is that $I=\omega_{Y}^{-1} F$ should square to -1 , in which case one can show that $I$ is an integrable complex structure.

This is a rather special condition, but there is a simple way to obey it that was important in [6] and will also be important in the present paper. We simply set $F=\omega_{J}$, in which case $\omega_{Y}^{-1} F=\omega_{K}^{-1} \omega_{J}$, which coincides with $I$ according to equation (2.2).

Thus, starting with the complex symplectic manifold $(Y, \Omega)$, for any choice of a unitary line bundle $\mathcal{L}$ with a connection of curvature $\omega_{J}=\operatorname{Re} \Omega$, we get an $A$-brane in the $A$-model of symplectic structure $\omega_{Y}$. We call this $A$-brane the canonical coisotropic brane and denote it as $\mathcal{B}_{\mathrm{cc}}$.

To make the $A$-model of symplectic form $\omega_{Y}$ concrete, it is usual to introduce an almost complex structure with respect to which $\omega_{Y}$ is positive and of type $(1,1)$. This enables one to develop a theory of pseudoholomorphic curves in $Y$, leading to an $A$-model that depends only on $\omega_{Y}$ and not on the chosen almost complex structure. There is no need for the almost complex structure to be integrable. In the present case, since the symplectic structure of the $A$-model is $\omega_{Y}=\omega_{K}$, it is natural to write $K$ for the almost complex structure that is used to define the $A$-model. 
To write the sigma-model action, one also uses the associated metric $g=-\omega_{K} K$. Furthermore, it is always possible to pick $K$ so that $I K=-K I$, implying that $J=K I$ is also an almost complex structure. $J$ will be useful in the quantization.

We stress that we make no assumption that $J$ and $K$ are integrable. A $K$ with the stated properties $\left(\omega_{K}\right.$ is of type $(1,1)$, and $I K=-K I$ ) always exists, and moreover the space of choices for $K$ is contractible. To see this, let $Y$ be of real dimension $4 n$. Let $S p(2 n)$ be the compact form of the symplectic group acting on $\mathbb{C}^{2 n}$, and $S p(2 n)_{\mathbb{C}}$ its complexification. The choice of $I, \Omega$ reduces the structure group of $Y$ from $G L(4 n, \mathbb{R})$ to $S p(2 n)_{\mathbb{C}}$. The further choice of $K$ reduces this group to $S p(2 n)$. (We have $S p(2 n)=U(2 n) \cap S p(2 n)_{\mathbb{C}}$, where $U(2 n)$ is the subgroup of $G L(4 n, \mathbb{R})$ that commutes with $K$.) As the quotient space $S p(2 n)_{\mathbb{C}} / S p(2 n)$ is contractible, a global choice of $K$ can be made, and there is no topology in the choice of $K$.

Though $J$ and $K$ need not be integrable, certainly the nicest case is that $Y$ admits a complete hyper-Kahler metric in which the three complex structures are $I, J$, and $K$ and the metric is $g=-\omega_{K} K$. (We call this a hyper-Kahler polarization.) In general, we cannot assume this, but many standard facts about the hyper-Kahler case are true in greater generality. For instance, it follows from $I K=-K I$ that $\omega_{J}$ is of type $(2,0) \oplus$ $(0,2)$ with respect to $K$. Indeed, the fact that $\omega_{K}$ is of type $(1,1)$ with respect to $K$ can be written $K^{t} \omega_{K} K=\omega_{K}$, which by (2.2) is the same as $-K^{t} I^{t} \omega_{J} K^{t}=\omega_{K}$, or $I^{t} K^{t} \omega_{J} K=\omega_{K}$. With $I^{2}=-1$ and using (2.2) again, this is $K^{t} \omega_{J} K=-\omega_{J}$, which is equivalent to saying that $\omega_{J}$ is of type $(2,0) \oplus(0,2)$ with respect to $K$. It now follows from $J=K I$ that $\omega_{J}$ is of type $(1,1)$ with respect to $J$. (We have $J^{t} \omega_{J} J=I^{t} K^{t} \omega_{J} K I=\omega_{J}$, since $\omega_{J}$ is of type $(2,0) \oplus(0,2)$ with respect to both $I$ and $K$.)

The relation between $J$ and $K$ is completely symmetrical; instead of beginning with $K$, and then defining $J$, we could have begun by introducing an almost complex structure $J$, constrained so that $I J=-J I$ and $\omega_{J}$ is of type $(1,1)$ with respect to $J$. The same argument as above would show existence of $J$, and then we could define $K=I J$, arriving at the same picture. As an example of this viewpoint, suppose we are given a middle-dimensional submanifold $M \subset Y$ such that $\omega_{J}$ is nondegenerate along $M$, and such that the tangent space $T Y$ to $Y$, when restricted to $M$, has a decomposition $T Y=T M \oplus I(T M)$, where $T M$ is the tangent space to $M$. Then we can constrain $J$ along $M$ so as to preserve the decomposition $T Y=T M \oplus I(T M)$; we simply define $J$ on $T M$ by picking an almost complex structure on $M$ such that $\omega_{J}$ is of type $(1,1)$, and then define $J$ on $I(T M)$ to ensure $I J=-J I$. Having defined $J$ along $M$, 
there is no topological obstruction to extending it over $Y$, again because $S p(2 n)_{\mathbb{C}} / S p(2 n)$ is contractible.

\subsection{Space of $\left(\mathcal{B}_{\mathrm{cc}}, \mathcal{B}_{\mathrm{cc}}\right)$ Strings}

Our next problem is to identify the algebra $\mathcal{A}$ of $\left(\mathcal{B}_{\mathrm{cc}}, \mathcal{B}_{\mathrm{cc}}\right)$ strings.

First we describe the space of $\left(\mathcal{B}_{\mathrm{cc}}, \mathcal{B}_{\mathrm{cc}}\right)$ strings additively. The space of $\left(\mathcal{B}_{\mathrm{cc}}, \mathcal{B}_{\mathrm{cc}}\right)$ strings in the $A$-model is the same as the space of operators that can be inserted in the $A$-model on a boundary of a string world-sheet $\Sigma$ that ends on the brane $\mathcal{B}_{\mathrm{cc}}$. So let us determine this.

We write $X$ for the bosonic fields in the sigma-model with target $Y$, and $\psi_{-}, \psi_{+}$for left- and right-moving fermionic fields. A boundary operator must be invariant under the supersymmetry (or BRST) symmetry of the $A$-model. The general $A$-model transformation law of $X$ is

$$
\delta X=(1-i K) \psi_{+}+(1+i K) \psi_{-} .
$$

Here we use an arbitrary almost complex structure $K$ (relative to which $\omega_{K}$ is of type $(1,1)$ and positive) in defining the $A$-model of symplectic structure $\omega_{K}$. A simple type of local operator is an operator $f(X)$ derived from a complex-valued function $f: Y \rightarrow \mathbb{C}$. For such an operator, inserted at an interior point of $\Sigma$, to be invariant under (2.3), $f$ must be constant.

However, we are interested in boundary operators, rather than bulk operators, and for this we must consider the boundary condition obeyed by the fermions. For a general space-filling rank 1 brane, this boundary condition is

$$
\psi_{+}=(g-F)^{-1}(g+F) \psi_{-} .
$$

In the present case, with $F=\omega_{J}$, we have $(g-F)^{-1}(g+F)=J$. So for boundary operators, $(2.3)$ collapses to $\delta X=((1-i K) J+(1+i K)) \psi_{-}$, which is equivalent to

$$
\delta X=(1+i I)(1+J) \psi_{-} .
$$

If we decompose $\delta X$ as $\delta^{1,0} X+\delta^{0,1} X$, where the two parts of the variation are of type $(1,0)$ and type $(0,1)$ with respect to $I$, then

$$
\begin{aligned}
& \delta^{1,0} X=0 \\
& \delta^{0,1} X=\rho,
\end{aligned}
$$


where $\rho=(1+i I)(1+J) \psi_{-}$. Clearly, since the square of the topological symmetry of the $A$-model vanishes, (2.6) implies that

$$
\delta \rho=0
$$

From (2.6), we see that for a string ending on the canonical coisotropic brane, a boundary operator $f(X)$ preserves the topological symmetry of the $A$-model if and only if the function $f$ is holomorphic in complex structure $I$. More generally, all boundary observables of the $A$-model can be constructed from $X$ and $\rho$, which have dimension 0 , since other fields have strictly positive dimension or vanish at the boundary. If we pick local complex coordinates on $Y$ corresponding to fields $X^{i}$, then a general operator of $q$ th order in $\rho$ takes the form $\rho^{\bar{i}_{1}} \rho^{\bar{i}_{2}} \ldots \rho^{\bar{i}_{q}} f_{\bar{i}_{1} \bar{i}_{2} \ldots \bar{i}_{q}}(X, \bar{X})$ and has charge $q$ under the ghost number symmetry of the $A$-model. By interpreting $\rho^{\bar{i}}$ as $d \bar{X}^{\bar{i}}$, we can interpret such an operator as a $(0, q)$-form on $Y$. Then it follows from (2.6) and (2.7) that the topological supercharge of the $A$-model corresponds to the $\bar{\partial}$ operator of $Y$.

So the observables of the $A$-model correspond additively to the graded vector space $H_{\bar{\partial}}^{0, \star}(Y)$ (where $Y$ is viewed as a complex manifold with complex structure $I$ ). For our purposes in this paper, we are mainly interested in the ghost number zero part of the ring of observables. Additively, this corresponds simply to the holomorphic functions on $Y$. However, the multiplicative structure is different. Classically, the holomorphic functions on $Y$ generate a commutative ring, but in the $A$-model, in the special case of a brane of type $\mathcal{B}_{\text {cc }}$, this ring is deformed to a noncommutative ring that we call $\mathcal{A}$. The deformation corresponds to deformation quantization using the Poisson brackets derived from the holomorphic symplectic two-form $\Omega$. One explanation of how this deformation comes about is given in Section 11.1 of $[6]$.

\subsection{Lagrangian brane and quantization}

So far, we have obtained an algebra $\mathcal{A}$ of $\left(\mathcal{B}_{\mathrm{cc}}, \mathcal{B}_{\mathrm{cc}}\right)$ strings. Now we want to find something (other than itself) that $\mathcal{A}$ can act on. The immediate answer is to introduce a second $A$-brane $\mathcal{B}^{\prime}$. Then $\mathcal{A}$ acts naturally on the space of $\left(\mathcal{B}_{\mathrm{cc}}, \mathcal{B}^{\prime}\right)$ strings (Figure 1$)$.

For this paper, we will consider $\mathcal{B}^{\prime}$ to be an $A$-brane of the simplest possible kind: a Lagrangian $A$-brane of rank 1 . Thus, $\mathcal{B}^{\prime}$ is supported on a Lagrangian submanifold $M$ and endowed with, roughly speaking, a flat 


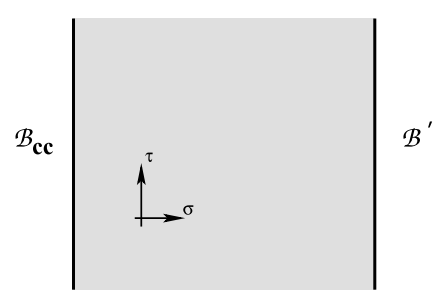

Figure 1: An open string with $\left(\mathcal{B}_{\mathrm{cc}}, \mathcal{B}^{\prime}\right)$ boundary conditions.

line bundle $\mathcal{L}^{\prime}$. The natural objects of study in what follows are branes, and it is important to bear in mind some subtleties about the relation between Lagrangian submanifolds and branes. First, because of disc instanton effects, not every pair $\left(M, \mathcal{L}^{\prime}\right)$ defines an $A$-brane. Second, in the absence of such effects, if two pairs $\left(M, \mathcal{L}^{\prime}\right)$ and $\left(M^{\prime}, \mathcal{L}^{\prime \prime}\right)$ differ by Hamiltonian isotopy, then the associated $A$-branes are equivalent. Finally, the interpretation of $\mathcal{L}^{\prime}$ as a flat line bundle is oversimplified, because of the relation of branes to $K$ theory and the role of the $B$-field. Each of these effects will play some role later.

To relate branes to quantization, we will impose a condition on $M$ that has no analog in the usual theory of the $A$-model. We assume that $\omega_{J}$ is nondegenerate when restricted to $M$. This is a mild condition in the sense that, acting on the tangent space to a given point in $Y, \omega_{J}$ is nondegenerate when restricted to a generic even-dimensional plane. Hence if the condition is true for a submanifold $M$, it is true for any sufficiently nearby submanifold. (The opposite case that $\omega_{J}$ is zero when restricted to $M$ was investigated in Section 11 of [6], and leads to $\mathcal{D}$-modules. We return to this in Section 3.9.)

If $\omega_{J}$ is nondegenerate when restricted to $M$, then the pair $\left(M, \omega_{J}\right)$ is a symplectic manifold, and this is the symplectic manifold that we will quantize.

As explained in Section 2.1, to define the $A$-model, we pick an almost complex structure $K$, with respect to which $\omega_{K}$ is of type $(1,1)$, and such that $I, K$, and $J=K I$ obey the algebra of quaternions. Once $M$ is given, it is convenient to further constrain $K$ such that the tangent bundle $T M$ is $J$-invariant. (That one can do so was explained at the end of Section 2.1.)

We now want to show that quantization of $\left(\mathcal{B}_{\mathrm{cc}}, \mathcal{B}^{\prime}\right)$ strings leads to quantization of the symplectic manifold $\left(M, \omega_{J}\right)$. To see this, we describe the sigma-model on a Riemann surface $\Sigma$, as in section 2.2 , by bosonic fields $X$ that describe the sigma-model map $\Phi: \Sigma \rightarrow Y$, and left and right-moving fermionic fields $\psi_{-}$and $\psi_{+}$. In general, the boundary conditions for fermions 
at the end of an open string are $\psi_{+}=R \psi_{-}$, for some matrix $R$. In the case of a space-filling brane of rank 1 , such as $\mathcal{B}_{\mathrm{cc}}$,

$$
R=(g-F)^{-1}(g+F) .
$$

Here $g$ is the metric of $Y$ and $F$ is the curvature of the Chan-Paton line bundle. For the brane $\mathcal{B}_{\mathrm{cc}}$, we have $F=\omega_{J}$ and $R=J$, so the boundary condition is

$$
\psi_{+}=J \psi_{-} .
$$

For a Lagrangian brane, supported on a submanifold $M, F=0$ but this does not mean that $R=1$. Rather, the boundary condition is

$$
\psi_{+}=\mathcal{R} \psi_{-},
$$

where $\mathcal{R}:\left.\left.T Y\right|_{M} \rightarrow T Y\right|_{M}$ is a reflection that leaves fixed the tangent bundle $T M$ to $M$, and acts as -1 on the normal bundle to $M$ (here $\left.T Y\right|_{M}$ is the tangent bundle of $Y$ restricted to $M$ ).

For our problem, we are principally interested in $\left(\mathcal{B}_{\mathrm{cc}}, \mathcal{B}^{\prime}\right)$ strings, that is strings that couple to $\mathcal{B}_{\mathrm{cc}}$ on the left and to $\mathcal{B}^{\prime}$ on the right. The boundary conditions are thus

$$
\begin{aligned}
\psi_{+}(0) & =J \psi_{-}(0) \\
\psi_{+}(\pi) & =\mathcal{R} \psi_{-}(\pi),
\end{aligned}
$$

where 0 and $\pi$ are the endpoints of the string. These boundary conditions do not allow $\psi$ to be constant along the string. For example, if $\psi$ is tangent to $M$ and also constant, the combination of the two boundary conditions gives $\psi_{+}=J \psi_{+}$, which since $\psi_{L}$ is real and $J^{2}=-1$ implies that $\psi_{+}=0$. Similarly, if $\psi$ is normal to $M$ and constant, we get $\psi_{+}=-J \psi_{+}$, again implying that $\psi_{+}=0$.

Now let us discuss the bosonic fields $X(\sigma, \tau)$, where $\sigma$ and $\tau$ are the worldsheet space and time coordinates. The bosonic fields do have zero modes, because the boundary condition $* d X=R d X$ is consistent with constant $X$; likewise the classical equations of motion are obeyed if $X$ is constant. The usual zero mode structure of the bosonic string is $X=x+p \tau+\ldots$, where $x$ is the zero mode, $p$ is its canonical momentum, and ... are the nonzero modes. Here, usually $x$ is canonically conjugate to $p$. In the present case, there is a constant $x$ term, but there is no $p \tau$ term in the expansion, because as soon as $X$ becomes $\tau$-dependent, the boundary condition $* d X=R d X$ at 
$\sigma=0$ does not permit $X$ to be independent of $\sigma$. Usually, in sigma-model perturbation theory, this effect can be treated perturbatively because $R$ is close to 1 ; but for a boundary associated with the brane $\mathcal{B}_{\text {cc }}$, we have $R^{2}=-1$, so there is no way to expand around $R=1$. The result is that the $p \tau$ term is missing in the expansion, which reads

$$
X=x+\ldots,
$$

where the omitted terms are nonzero modes. The nonzero modes are, of course, related by worldsheet supersymmetry to the nonzero modes of $\psi$. For a detailed explanation of this expansion in an example, see [14].

In the context of quantizing the $\left(\mathcal{B}_{\mathrm{cc}}, \mathcal{B}^{\prime}\right)$ strings, in addition to the boundary condition at $\sigma=0$, there is also a boundary condition $X(\pi) \in M$ at the other end. Because of this boundary condition, the zero modes in (2.12) take values in $M$.

So it must be that the components of $x$ are canonically conjugate to each other. The reason that this happens is that the action of a string ending on a brane with Chan-Paton connection $A$ contains a boundary term $\int_{\partial \Sigma} A_{\mu} d X^{\mu}$. In the case of $\left(\mathcal{B}_{\mathrm{cc}}, \mathcal{B}^{\prime}\right)$ strings, the Chan-Paton bundle $\mathcal{L}^{\prime}$ of the brane $\mathcal{B}^{\prime}$ is flat, while the Chan-Paton bundle of $\mathcal{B}_{\mathrm{cc}}$ is the unitary line bundle $\mathcal{L}$ of curvature $\omega_{J}$. We write $A$ and $A^{\prime}$ for the connections on these two line bundles. Classically, the action for the zero modes is $\int d \tau\left(A_{\mu}-A_{\mu}^{\prime}\right) d x^{\mu} / d \tau$, where the two terms come from the left and right endpoints of the string. We define a line bundle $\mathcal{N}=\mathcal{L} \otimes\left(\mathcal{L}^{\prime}\right)^{-1}$ over $M$. $\mathcal{N}$ is a unitary line bundle with a connection $B=A-A^{\prime}$ of curvature $\omega_{J}$. The action for the zero modes is, in this approximation,

$$
\int B_{\mu} \frac{d x^{\mu}}{d \tau} d \tau .
$$

Formally speaking, to quantize the zero modes with this action (which actually has an important "quantum" correction that we will describe shortly) amounts to quantizing $M$ with prequantum line bundle $\mathcal{N}$. Just knowing this does not give any general solution to the problem of quantization. All we learn is that, if the $A$-model of $Y$ exists, then the space of $\left(\mathcal{B}_{\mathrm{cc}}, \mathcal{B}^{\prime}\right)$ strings can be understood as the result of quantizing $M$ with prequantum line bundle $\mathcal{N}$. If the $A$-model exists, and the two boundary conditions associated with branes $\mathcal{B}_{\mathrm{cc}}$ and $\mathcal{B}^{\prime}$ also exist, then the space of $\left(\mathcal{B}_{\mathrm{cc}}, \mathcal{B}^{\prime}\right)$ strings exists even if it is hard to describe this space explicitly.

Usually, given two $A$-branes or $B$-branes $\mathcal{B}_{1}$ and $\mathcal{B}_{2}$, one can go to a large volume limit and describe the space of $\left(\mathcal{B}_{1}, \mathcal{B}_{2}\right)$ strings in terms of classical 
geometry. The delicate structure of the coisotropic brane $\mathcal{B}_{\mathrm{cc}}$ prevents us from doing this successfully for the $\left(\mathcal{B}_{\mathrm{cc}}, \mathcal{B}^{\prime}\right)$ strings, in general.

But we can use general properties of the $A$-model to learn general properties of quantization. One can also get some information from classical geometry, as we explain next.

Branes of type $(A, B, A)$

There is an important special case in which we can describe explicitly the space of $\left(\mathcal{B}_{\mathrm{cc}}, \mathcal{B}^{\prime}\right)$ strings. This is the case that we are given a hyper-Kahler polarization of $(Y, M)$. This is a hyper-Kahler structure on $Y$, extending its complex symplectic structure $(I, \Omega)$, such that $M$ is a complex submanifold in complex structure $J$.

Under these conditions, the branes $\left(\mathcal{B}_{\mathrm{cc}}, \mathcal{B}^{\prime}\right)$ are both branes of type $(A, B, A)$, that is, $A$-branes for the $A$-models with symplectic forms $\omega_{I}$ or $\omega_{K}$, and $B$-branes for the $B$-model of complex structure $J$. For example, $\mathcal{B}_{\text {cc }}$ is a $B$-brane of type $J$ because the curvature form $\omega_{J}$ of its ChanPaton line bundle is of type $(1,1)$ in complex structure $J$. Similarly, $\mathcal{B}^{\prime}$ is a $B$-brane because $M$ is a complex submanifold and the Chan-Paton bundle of $\mathcal{B}^{\prime}$ is flat.

We can now look at the space of $\left(\mathcal{B}_{\text {cc }}, \mathcal{B}^{\prime}\right)$ strings in two different ways. We defined $\mathcal{H}$ to be the space of $\left(\mathcal{B}_{\mathrm{cc}}, \mathcal{B}^{\prime}\right)$ strings in the $A$-model of $\omega_{Y}=\omega_{K}$. Similarly, we can define $\tilde{\mathcal{H}}$ to be the space of $\left(\mathcal{B}_{\mathrm{cc}}, \mathcal{B}^{\prime}\right)$ strings in the $B$-model of complex structure $J$. As long as $M$ is compact or wavefunctions are required to vanish sufficiently rapidly at infinity, the two spaces are the same, since they both can be described as the space of zero energy states of the sigma-model with target $Y$ (compactified on an interval with boundary conditions at the two ends determined by $\mathcal{B}_{\mathrm{cc}}$ and $\left.\mathcal{B}^{\prime}\right)$.

One qualification is that the equivalence of $\mathcal{H}$ and $\tilde{\mathcal{H}}$ does not respect their grading. $\mathcal{H}$ and $\tilde{\mathcal{H}}$ are both $\mathbb{Z}$-graded, because the $A$-model of type $\omega_{K}$ and the $B$-model of type $J$ are both $\mathbb{Z}$-graded by "ghost number." The gradings are different, but conjugate. In fact, the sigma-model of target $Y$, with boundary conditions set by two branes of type $(A, B, A)$, has an $S U(2)$ group of $R$-symmetries that we call $S U(2)_{R}$; the two ghost number symmetries are conjugate but different $U(1)$ subgroups of $S U(2)_{R}$. In practice, however, one usually studies quantization in a situation in which the grading is trivial; this is so if $\mathcal{N}$ is very ample as a line bundle in complex structure $J$.

To use the equivalence between $\mathcal{H}$ and $\tilde{\mathcal{H}}$, we need to have a way to determine $\tilde{\mathcal{H}}$. For this, we simply observe that in the $B$-model, the choice 
of Kahler metric is irrelevant. So we can rescale the metric of $Y$ by a factor $t \gg 1$, reducing to a situation in which sigma-model perturbation theory is valid. In this limit, by a standard argument, we describe $\tilde{\mathcal{H}}$ by $\bar{\partial}$ cohomology:

$$
\tilde{\mathcal{H}}=\oplus_{i=0}^{\operatorname{dim}_{\mathbb{C}} M} H^{i}\left(M, K^{1 / 2} \otimes \mathcal{N}\right) .
$$

Since $\mathcal{H} \cong \tilde{\mathcal{H}}$, we can express this as a statement about $\mathcal{H}$ :

$$
\mathcal{H}=\oplus_{i=0}^{\operatorname{dim}_{\mathbb{C}} M} H^{i}\left(M, K^{1 / 2} \otimes \mathcal{N}\right) .
$$

Here, roughly speaking, $K^{1 / 2}$ is the square root of the canonical line bundle $K$ of $M$. (A more precise explanation is given presently.) For very ample $\mathcal{N}$, the cohomology vanishes except for $i=0$ and its $\mathbb{Z}$-grading is trivial.

The description (2.15) of $\mathcal{H}$ has an important limitation, beyond the problem with the grading. It describes $\mathcal{H}$ as a vector space, but it does not lead to a natural description of the Hilbert space structure of $\mathcal{H}$, when there is one. In Section 2.4, we will describe the conditions under which $\mathcal{H}$ has a hermitian inner product that can be defined in the $A$-model. Analogous but different conditions ${ }^{3}$ lead to a natural hermitian inner product on $\tilde{\mathcal{H}}$ in the $B$-model. The two hermitian structures are different (when they both exist) and the equivalence between $\mathcal{H}$ and $\tilde{\mathcal{H}}$ does not map a natural hermitian structure of the $A$-model to a natural structure of the $B$-model. (This identification does preserve a third hermitian product, the one that $\mathcal{H}$ and $\tilde{\mathcal{H}}$ get from their interpretation as the space of physical ground states in the sigma-model with hyper-Kahler metric. This one is not natural in the $A$-model or the $B$-model and is not visible in the large volume limit that leads to (2.14).) Hence, when applicable, (2.15) describes $\mathcal{H}$ as a vector space, not as a vector space with a hermitian inner product.

The description of $\mathcal{H}$ that we have just given has an obvious resemblance to a standard statement in geometric quantization. In that context, the choice of an integrable complex structure $J$ on $M$, such that the symplectic form of $M$ becomes a Kahler form, is known as a complex polarization. Equation (2.15) then defines quantization with a complex polarization.

A hyper-Kahler polarization of the pair $(Y, M)$, which we used in the above derivation, plays an analogous role in our approach. Our statement is that for any choice of hyper-Kahler polarization, the space of $\left(\mathcal{B}_{\mathrm{cc}}, \mathcal{B}^{\prime}\right)$ strings can be described as in (2.15).

\footnotetext{
${ }^{3}$ To define a hermitian metric in the $B$-model, one uses an involution $\tau$ of $Y$ that maps $M$ to itself and reverses the sign of $J$, while in the $A$-model, $\tau$ reverses the sign of $\omega_{Y}$, as explained in Section 2.4.
} 
There are two primary differences between our statement and the analogous statement in geometric quantization:

(1) In our framework, the space of $\left(\mathcal{B}_{\mathrm{cc}}, \mathcal{B}^{\prime}\right)$ strings is an $A$-model invariant and therefore is independent of the choice of a hyper-Kahler polarization. In geometric quantization, there is no general statement about when the right-hand side of (2.14) is independent of the choice of complex polarization $J$.

(2) In our framework, (2.14) is a statement about vector spaces, while in geometric quantization, one usually endows the right-hand side of (2.14) with a hermitian structure. For example, in the very ample case, one defines a Hilbert space norm by $|\psi|^{2}=\int_{M}\left(\omega^{n} / n !\right) \bar{\psi} \psi$, where $\omega$ is the symplectic form of $M, 2 n$ is the real dimension of $M$, and $\psi \in H^{0}\left(M, K^{1 / 2} \otimes \mathcal{N}\right)$. This is certainly a natural formula in Kahler geometry (it describes the hermitian metric that arises in the sigmamodel after rescaling the metric of $Y$ by a factor $t \gg 1$ ), but it is not a natural $A$-model inner product. A somewhat related statement is that in our framework, (2.14) does not describe the natural $\mathbb{Z}$-grading of $\mathcal{H}$, but a conjugate one.

\section{Relation to K-theory}

A point that still remains to be clarified is the meaning of the symbol $K^{1 / 2}$ in the above analysis. In general, $M$ may not be a spin manifold, so a line bundle $K^{1 / 2}$ may not exist, and $M$ may not be simply connected, so that if $K^{1 / 2}$ exists, it may not be unique up to isomorphism.

The resolution of this point depends upon the relation of branes to $K$ theory. The following are general statements about branes, independent of any specialization to an $A$-model or a $B$-model. Consider a brane supported on a submanifold $N \subset Y$ and endowed with a rank 1 Chan-Paton bundle $\mathcal{T}$. Naively, $\mathcal{T}$ is a complex line bundle, but actually, because of an anomaly in the sigma-model [24], $\mathcal{T}$ can be more precisely described as a $\operatorname{Spin}_{\mathrm{c}}$ structure on the normal bundle to $N$ in $Y$.

For the space-filling brane $\mathcal{B}_{\text {cc }}, N$ is equal to $Y$, so the normal bundle to $N$ is trivial. Hence the Chan-Paton bundle $\mathcal{L}$ of $\mathcal{B}_{\text {cc }}$ is an ordinary complex line bundle. For the Lagrangian $A$-brane $\mathcal{B}^{\prime}, N$ is the Lagrangian submanifold $M$. The tangent bundle and normal bundle to a Lagrangian submanifold are naturally isomorphic (under multiplication by $\omega_{Y}=\omega_{K}$ ), so the ChanPaton "line bundle" $\mathcal{L}^{\prime}$ of $\mathcal{B}^{\prime}$ is really a choice of $\operatorname{Spin}_{\mathrm{c}}$ structure on $M$. If $\mathcal{L}^{\prime}$ were actually a line bundle, there would be no natural line bundle $\left(\mathcal{L}^{\prime}\right)^{-1} \otimes K^{1 / 2}$, since $K^{1 / 2}$ may not exist and may not be unique. However, for $\mathcal{L}^{\prime}$ a $\operatorname{Spin}_{\text {c }}$ structure, there is a natural line bundle $\left(\mathcal{L}^{\prime}\right)^{-1} \otimes K^{1 / 2}$; the 
two factors in this tensor product are not naturally defined as complex line bundles, but the tensor product is.

Now the meaning of (2.14) is clear. With $\mathcal{N}=\mathcal{L} \otimes\left(\mathcal{L}^{\prime}\right)^{-1}$, and $\mathcal{L}$ an ordinary complex line bundle, there is no problem in defining $\mathcal{N} \otimes K^{1 / 2}$ as a complex line bundle, though the two factors separately do not have this status.

In this discussion, we did not assume that the rank 1 brane supported on $N$ is supposed to be an $A$-brane or a $B$-brane. If this brane is supposed to be an $A$-brane supported on a Lagrangian submanifold $M$, then $\mathcal{L}^{\prime}$ must be a flat $\operatorname{Spin}_{\mathrm{c}}$ structure on $M$. There is a topological obstruction to hav-

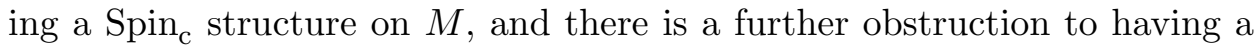
flat $\operatorname{Spin}_{\mathrm{c}}$ structure. In general, $\mathrm{Spin}_{\mathrm{c}}$ structures on $M$ are classified topologically by the choice of a way of lifting the second Stieffel-Whitney class $w_{2}(M) \in H^{2}\left(M, \mathbb{Z}_{2}\right)$ to an integral cohomology class $\zeta \in H^{2}(M, \mathbb{Z})$. Flat

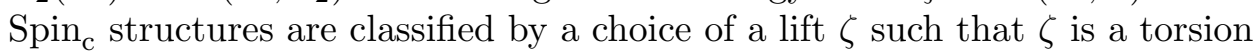
element of $H^{2}(M, \mathbb{Z})$. In general, even if $M$ is $\operatorname{Spin}_{\mathrm{c}}$, it may not admit a flat $\operatorname{Spin}_{\mathrm{c}}$ structure, since it may be impossible to pick the lift $\zeta$ to be a torsion class. A symplectic manifold that does not admit a flat $\operatorname{Spin}_{\mathrm{c}}$ structure cannot be quantized in our sense.

This obstruction to quantization has been encountered in the literature on representations of a semi-simple noncompact real Lie group $G$. In that context, $M$ is a coadjoint orbit of $G$, and one aims to obtain a representation of $G$ by quantization of $M$. (See Section 3 for more on this.) This problem has been approached from many different points of view. In [20], which is perhaps the closest to the approach in the present paper, a preliminary step to quantizing $M$ is to, in effect, endow $M$ with a flat $\operatorname{Spin}_{\mathrm{c}}$ structure. For example, the minimal orbit of $S O(p, q)$ with $p+q$ odd and $p, q \geq 4$ does not admit such a structure and cannot be quantized by the methods of [20] or of the present paper.

General shift by $K^{1 / 2}$

Now let us return to the zero mode action (2.13),

$$
\int B_{\mu} \frac{d x^{\mu}}{d \tau} d \tau
$$

dropping the assumption of a hyper-Kahler polarization. In the original derivation, $B$ emerged as a connection on the "complex line bundle" $\mathcal{N}=$ $\mathcal{L} \otimes\left(\mathcal{L}^{\prime}\right)^{-1}$. However, as we have just explained, in general $\mathcal{N}$ does not make sense as a complex line bundle. 
Simply to make sense of the formula, there must be a correction that shifts $\mathcal{N}$ to $\hat{\mathcal{N}}=\mathcal{L} \otimes\left(\mathcal{L}^{\prime}\right)^{-1} \otimes K^{1 / 2}$ (or something similar to this), where $K$ is the canonical line bundle ${ }^{4}$ of $M$. The connection that appears in the action must be a connection on $\hat{\mathcal{N}}$.

The way that this correction arises is as follows. As a step toward quantizing the open strings with $\left(\mathcal{B}_{\mathrm{cc}}, \mathcal{B}^{\prime}\right)$ boundary conditions, one quantizes the worldsheet fermions, expanding around a map from the string worldsheet to $Y$ that consists of a constant map to a point $p \in M$. In expanding around such a constant map, there are no fermion zero modes, since they are all removed by the boundary condition (2.11). Hence, the space of ground states in the fermion Fock space is a one-dimensional vector space $W_{p}$. As $p$ varies, $W_{p}$ varies as the fiber of a complex "line bundle" $\mathcal{W} \rightarrow M . \mathcal{W}$ and the induced connection on it must be included as an additional factor in quantizing the bosonic zero modes.

In fact, $\mathcal{W}$ is isomorphic to $K^{1 / 2}$ (and is not quite well-defined as a line bundle because of an anomaly in the relevant family of fermion Fock spaces). One can show that $\mathcal{W} \cong K^{1 / 2}$ by using standard methods to determine the quantum numbers of the Fock space ground state. This result is also needed to match the $B$-model analysis in the case that one uses a hyper-Kahler polarization.

\subsection{Unitarity}

So far, to an $A$-brane $\mathcal{B}^{\prime}$, we have associated a vector space $\mathcal{H}$ consisting of $\left(\mathcal{B}_{\mathrm{cc}}, \mathcal{B}^{\prime}\right)$ strings. Quantum mechanics usually involves Hilbert spaces, however, and the question arises of how to define a hermitian inner product on $\mathcal{H}$.

For this, we want to make a general analysis of how a hermitian inner product can appear in the topological $A$-model. In this general discussion, we are only concerned with the topological $A$-model with symplectic structure $\omega_{K}$. (Additional structures such as $I$ and $\omega_{J}$ are not relevant.)

If $\mathcal{B}_{1}$ and $\mathcal{B}_{2}$ are two branes in the topological $A$-model, there is always a natural duality between the space of $\left(\mathcal{B}_{1}, \mathcal{B}_{2}\right)$ strings and the space of

\footnotetext{
${ }^{4}$ The metric on $Y$ that is used to define the $A$-model, even if it is not hyper-Kahler, restricts to a metric on $M$. The metric on $M$, together with the symplectic structure, determines an almost complex structure on $M$, and this almost complex structure is used to define $K$.
} 
$\left(\mathcal{B}_{2}, \mathcal{B}_{1}\right)$ strings. It is given by the two point function on the disc. Consequently, defining a hermitian inner product on the space of $\left(\mathcal{B}_{\mathrm{cc}}, \mathcal{B}^{\prime}\right)$ strings is equivalent to finding a complex antilinear map from $\left(\mathcal{B}_{\mathrm{cc}}, \mathcal{B}^{\prime}\right)$ strings to $\left(\mathcal{B}^{\prime}, \mathcal{B}_{\mathrm{cc}}\right)$ strings.

Let us start by assuming that our $A$-model is a twisted version of a physically sensible, unitary, supersymmetric field theory. In general, any such field theory has an antilinear CPT symmetry, which we will denote $\Theta$. For any pair $\mathcal{B}_{1}, \mathcal{B}_{2}$, the transformation $\Theta$ maps $\left(\mathcal{B}_{1}, \mathcal{B}_{2}\right)$ strings to $\left(\mathcal{B}_{2}, \mathcal{B}_{1}\right)$ strings. This gives an antilinear map from $\left(\mathcal{B}_{\mathrm{cc}}, \mathcal{B}^{\prime}\right)$ strings to $\left(\mathcal{B}^{\prime}, \mathcal{B}_{\mathrm{cc}}\right)$ strings, but it cannot be the map we want, because it is not a symmetry of the $A$-model. The definition of the $A$-model depends on a choice of a differential $Q$, which is a complex linear combination of the supercharges. CPT maps $Q$ to $Q^{\dagger}$ (its hermitian adjoint), which is the differential of a complex conjugate $A$-model.

To get a symmetry of the $A$-model, we need to combine $\Theta$ with a transformation that maps the conjugate $A$-model back to the original $A$-model. We get such a transformation if $Y$ admits an involution $\tau$ (that is a diffeomorphism obeying $\tau^{2}=1$ ) with the property that $\omega_{K}$ is odd under $\tau$ :

$$
\tau^{*} \omega_{K}=-\omega_{K} .
$$

This property implies that $\tau$ maps the $A$-model to the conjugate $A$-model. In fact, we can always pick a $\tau$-invariant metric $g$, such that $\omega_{K}$ is of type $(1,1)$. Then the almost complex structure $K=g^{-1} \omega_{K}$ is odd under $\tau$. So $\tau$ maps pseudoholomorphic curves to pseudo-antiholomorphic curves, and thus maps the $A$-model to the conjugate $A$-model.

So $\Theta_{\tau}=\tau \Theta$ is an antilinear map from the $A$-model to itself. This is a general statement about the $A$-model and holds whether or not the $A$-model can be obtained by twisting an underlying physical theory. (The latter is possible if one can choose $K$ to be integrable.)

Now if $\mathcal{B}_{1}$ and $\mathcal{B}_{2}$ are $\tau$-invariant $A$-branes, we can use $\Theta_{\tau}$ to define a hermitian inner product on the space $\mathcal{H}$ of $\left(\mathcal{B}_{1}, \mathcal{B}_{2}\right)$ strings. If $($,$) is the$ pairing between $\left(\mathcal{B}_{1}, \mathcal{B}_{2}\right)$ strings and $\left(\mathcal{B}_{2}, \mathcal{B}_{1}\right)$ strings given by the two-point function on the disc, then the inner product on $\mathcal{H}$ is given by

$$
\left\langle\psi, \psi^{\prime}\right\rangle=\left(\Theta_{\tau} \psi, \psi^{\prime}\right) .
$$

Now let us apply this to our problem. For the brane $\mathcal{B}_{\text {cc }}$ to be $\tau$-invariant, we first of all need that the curvature $\omega_{J}$ of the Chan-Paton bundle of this 
brane should be $\tau$-invariant:

$$
\tau^{*} \omega_{J}=\omega_{J}
$$

We also actually need a little more: the action of $\tau$ on $M$ should lift to an action on the Chan-Paton line bundle $\mathcal{L}$, whose curvature is $\omega_{J}$, and we must pick such a lift. Since $\omega_{K}$ is odd under $\tau$ while $\omega_{J}$ is even, it follows that $\tau$ is antiholomorphic from the point of view of the complex structure $I=\omega_{J}^{-1} \omega_{K}$ :

$$
\tau^{*} I=-I
$$

It follows from this that each component of the fixed point set of $\tau$ is middle-dimensional. With $\Omega=\omega_{J}+i \omega_{K}$, we also have

$$
\tau^{*} \Omega=\bar{\Omega} \text {. }
$$

So more briefly, we can summarize the above conditions by saying that $\tau$ is an antiholomorphic involution of the complex symplectic manifold defined by the data $(Y, I, \Omega)$, with a lift to $\mathcal{L}$.

For the Lagrangian $A$-brane $\mathcal{B}^{\prime}$ to be $\tau$-invariant, its support $M$ (and its Chan-Paton line bundle $\mathcal{L}^{\prime}$ ) must be $\tau$-invariant, and again, we need a lift of $\tau$ to act on $\mathcal{L}^{\prime}$. In this case, we get, finally, a hermitian form on the space $\mathcal{H}$ of $\left(\mathcal{B}_{\mathrm{cc}}, \mathcal{B}^{\prime}\right)$ strings. Note that $\tau$ need not leave $M$ fixed pointwise, but it must map $M$ to itself. If $\tau$ acts trivially on $M$, the lift to $\mathcal{L}^{\prime}$, if it exists, can be uniquely specified by saying that $\tau$ acts trivially on the restriction of $\mathcal{L}^{\prime}$ to $M$. If $\tau$ acts nontrivially on $M$, its lift to $\mathcal{L}^{\prime}$ may involve a subtle choice.

The hermitian inner product $\langle$,$\rangle is not necessarily positive definite (which$ means as usual that $\langle\psi, \psi\rangle>0$ for all nonzero $\psi \in \mathcal{H}$ ), but it is always nondegenerate. Nondegeneracy means that given $\psi \in \mathcal{H}$, there is always $\chi \in$ $\mathcal{H}$ such that $\langle\chi, \psi\rangle \neq 0$. This follows from nondegeneracy of the underlying topological inner product $($,$) and the fact that \Theta_{\tau}^{2}=1$. Picking $\chi_{0}$ such that $\left(\chi_{0}, \psi\right) \neq 0$ and setting $\chi=\Theta_{\tau} \chi_{0}$, we have $\langle\chi, \psi\rangle \neq 0$.

Near the classical limit, the norm of a state $\psi \in \mathcal{H}$ is roughly $\langle\psi, \psi\rangle=$ $\int_{M} \bar{\psi}(\tau x) \psi(x)$. Such a form can be positive definite only if $\tau$ acts trivially on $M$. So for $\langle$,$\rangle to be positive near the classical limit, we require that \tau$ should act trivially on $M$. Since $M$ (being Lagrangian) is middle-dimensional, while the fixed point set of the antiholomorphic involution $\tau$ is also middledimensional, this means that $M$, assuming it is connected, is a component of the fixed point set. (In Section 3.8, we give an example far from the classical limit in which $\langle$,$\rangle is positive even though \tau$ acts nontrivially on $M$.) 
Conversely, if $M$ is a component of the fixed point set of $\tau$, then, as $\omega_{K}$ is odd under $\tau, M$ is automatically Lagrangian for $\omega_{K}$. Moreover, $\omega_{J}$ is automatically nondegenerate when restricted to $M$. (To prove this, start with the fact that, if $M$ is held fixed by an antiholomorphic involution $\tau$, then the restriction to $M$ of the tangent bundle to $Y$ decomposes as $\left.T Y\right|_{M}=T M \oplus I(T M) . \tau$ acts as 1 and -1 on the two summands, and, being $\tau$-invariant, $\omega_{J}$ is the sum of a nondegenerate two-form on $T M$ and one on $I(T M)$.)

Thus, the case of our construction that leads to unitarity near the classical limit is much more specific. Introducing the space of $\left(\mathcal{B}_{\mathrm{cc}}, \mathcal{B}_{\mathrm{cc}}\right)$ strings, we carry out deformation quantization of the complex symplectic manifold $Y$, constructing an associative algebra $\mathcal{A}$. Then, to get a Hilbert space $\mathcal{H}$ on which $\mathcal{A}$ acts, we pick an antiholomorphic involution $\tau$ (with a lift to the line bundle $\mathcal{L} \rightarrow Y$ ) and a component $M$ of the fixed point set supporting a $\tau$-invariant $A$-brane $\mathcal{B}^{\prime} . \mathcal{H}$ is defined as the space of $\left(\mathcal{B}_{\mathrm{cc}}, \mathcal{B}^{\prime}\right)$ strings, and $\mathcal{A}$ acts on $\mathcal{H}$.

The operation $\Theta_{\tau}$ acts on a function $f$ on $Y$, defining a $\left(\mathcal{B}_{\mathrm{cc}}, \mathcal{B}_{\mathrm{cc}}\right)$ string, as the composition of $\tau$ with complex conjugation. So if $\mathcal{O}_{f}: \mathcal{H} \rightarrow \mathcal{H}$ is the operator associated to $f$, then the hermitian adjoint of $\mathcal{O}_{f}$ is associated with the function $\tau(\bar{f})$. In particular, if $\tau$ leaves $M$ fixed pointwise and $f$ is real when restricted to $M$, then $\tau(\bar{f})=f$ and $\mathcal{O}_{f}$ is hermitian.

\section{$3 \quad$ Branes and representations}

\section{$3.1 \quad$ An example}

To make these ideas more concrete, we will consider in more depth an example that was introduced in Section 1.2. This is the case that $Y$ is an affine algebraic variety defined by the equation $x^{2}+y^{2}+z^{2}=\mu^{2} / 4$ in complex variables $x, y, z$. Here $\mu^{2}$ is a complex constant. $Y$ admits the action of a group $S O(3, \mathbb{C})$, rotating $x, y$, and $z$. In quantization, we will encounter the double cover of $S O(3, \mathbb{C})$, which is $S L(2, \mathbb{C})$.

We make $Y$ a complex symplectic manifold by introducing the holomorphic two-form

$$
\Omega=h^{-1} \frac{d y \wedge d z}{x},
$$

where $h$ is another complex constant. $h$ could be eliminated (at the cost of changing $\mu$ ) by rescaling $x, y$, and $z$, and we will eventually set $h=1$. 
$\Omega$ is $S O(3, \mathbb{C})$-invariant. One way to show this is to observe that $Y$ is defined by the equation $f=0$ in $\mathbb{C}^{3}$, where $f=x^{2}+y^{2}+z^{2}-\mu^{2} / 4$. The meromorphic differential $2 h^{-1} d x \wedge d y \wedge d z / f$ is manifestly $S O(3, \mathbb{C})$ invariant, with a pole precisely at $f=0$. The residue of this pole is $\Omega$.

Consequently, the $A$-model of $Y$ with symplectic structure $\omega_{Y}=\operatorname{Im} \Omega$ has $S O(3, \mathbb{C})$ symmetry. If we consider only the usual Lagrangian $A$-branes, we do not get much from the $S O(3, \mathbb{C})$ action. That action becomes interesting, however, if we introduce coisotropic $A$-branes.

As a preliminary, we compute the cohomology class of $\Omega$. We set $x=$ $\hat{x} \sqrt{\mu^{2}} / 2, y=\hat{y} \sqrt{\mu^{2}} / 2, z=\hat{z} \sqrt{\mu^{2}} / 2$. The two-dimensional homology of $Y$ is generated by the real two-cycle $S$ defined by $\hat{x}^{2}+\hat{y}^{2}+\hat{z}^{2}=1$. We compute that

$$
\int_{S} \frac{\Omega}{2 \pi}=h^{-1} \mu
$$

where $\mu= \pm \sqrt{\mu^{2}}$. The sign depends on the orientation of $S$.

Hence the cohomology class of $\operatorname{Re} \Omega / 2 \pi$ is integral if $n=\operatorname{Re}\left(h^{-1} \mu\right)$ is an integer. Under this condition, we can construct a unitary line bundle $\mathcal{L}$ with a connection of curvature $\operatorname{Re} \Omega$. This determines a space-filling coisotropic brane $\mathcal{B}_{\mathrm{cc}}$ in the $A$-model with symplectic structure $\omega_{Y}=\operatorname{Im} \Omega$. The first Chern class of $\mathcal{L}$, integrated over $S$, is equal to $n$ :

$$
\int_{S} c_{1}(\mathcal{L})=n .
$$

Now let us describe the $\operatorname{ring} \mathcal{A}$ of $\left(\mathcal{B}_{\mathrm{cc}}, \mathcal{B}_{\mathrm{cc}}\right)$ strings. Classically, as explained in Section 1.2, it is the commutative ring of polynomial functions in $x, y$, and $z$, modulo the commutativity relation

$$
[x, y]=[y, z]=[z, x]=0
$$

and the geometrical relation

$$
x^{2}+y^{2}+z^{2}=\mu^{2} / 4 .
$$

In the context of the coisotropic brane, we have to consider possible corrections to those relations. Corrections involve the Poisson brackets, which are proportional to $\Omega^{-1}=h z(d x \wedge d y)^{-1}$, and higher order terms involving higher powers of $\Omega^{-1}$ and its derivatives. 
It is simple to determine the corrections to the classical relations, because they are severely constrained by (i) $S O(3, \mathbb{C})$ symmetry, (ii) holomorphy, and (iii) an approximate scaling symmetry. Of these points, $S O(3, \mathbb{C})$ symmetry requires little explanation. By holomorphy, we mean holomorphy in $h$ and $\mu^{2}$ near $h=\mu^{2}=0$. Holomorphy in $h$ is manifest in sigma-model perturbation theory. At $\mu^{2}=0, Y$ develops a singularity at the origin, but the ring $\mathcal{A}$ is nevertheless holomorphic in $\mu^{2}$ since the corrections to the classical ring structure can be computed in a region of field space far away from the singularity of $Y$. Finally, if $\mu^{2}=0$, then $Y$ has a scaling symmetry $(x, y, z) \rightarrow(t x, t y, t z), t \in \mathbb{C}^{*}$.

For $\mu^{2} \neq 0$, this is only an asymptotic symmetry, valid for $(x, y, z) \rightarrow$ $\infty$. Even if $\mu^{2}=0$, the algebra $\mathcal{A}$ does not have the scaling symmetry, because $\Omega$ does not possess this symmetry. Still, the scaling symmetry heavily constrains the corrections to the classical algebra generated by $x, y$, and $z$. Under the scaling symmetry, we have $\mu^{2} \rightarrow t^{2} \mu^{2}, h \rightarrow t h$. The crucial point is that $\mu^{2}$ and $h$ both scale with positive powers of $t$.

The most general deformation of (3.4) that is allowed by holomorphy and $S O(3, \mathbb{C})$ symmetry is $[x, y]=f\left(\mu^{2}, h\right) z$ (and cyclic permutations) for some function $f$. Under scaling, $f$ must be of degree 1 , and holomorphy then implies that it is linear in $h$ and independent of $\mu^{2}$. But the term linear in $h$ can be computed simply from the classical Poisson bracket, giving $[x, y]=h z$, and cyclic permutations.

As for the relation $(3.5)$, holomorphy, $S O(3, \mathbb{C})$ symmetry, and scaling symmetry dictate that $h$-dependent corrections can only take the form of a constant multiple of $h^{2}$. Thus the relation must take the form $x^{2}+y^{2}+z^{2}=$ $\mu^{2} / 4+c h^{2}$, for some complex constant $c$. This constant can be computed by going to second order in sigma-model perturbation theory, but instead we will use an indirect method to show in Section 3.2 that $c=-1 / 4$.

Because of the scaling symmetry, we lose nothing if we set $h=1$. The form of the algebra is then that $x, y$, and $z$ obey the $S O(3, \mathbb{C})$ commutation relations

$$
[x, y]=z,[y, z]=x,[z, x]=y,
$$

and that the quadratic Casimir operator $J^{2}=x^{2}+y^{2}+z^{2}$ is

$$
J^{2}=\frac{\mu^{2}-1}{4} .
$$

For an alternative way to compute this algebra, from a different starting point, see [25]. 
The preceding statements imply that if $\mathcal{B}^{\prime}$ is any $A$-brane, then the space of $\left(\mathcal{B}_{\text {cc }}, \mathcal{B}^{\prime}\right)$ strings has a natural action of the Lie algebra $\mathfrak{s o}(3, \mathbb{C})$ or $\mathfrak{s l}(2, \mathbb{C})$ (generated by $x, y, z)$. The value of the quadratic Casimir is $\left(\mu^{2}-1\right) / 4$, independent of the choice of $\mathcal{B}^{\prime}$. We will see examples below; with different choices of $\mathcal{B}^{\prime}$, we will get different $\mathfrak{s l}(2, \mathbb{C})$-modules, all with the same value of the quadratic Casimir.

To get a natural group action (as opposed to an action of the Lie algebra) requires more. The reason for this is that if $G$ is a noncompact group, then a representation of the Lie algebra $\mathfrak{g}$ of $G$ does not automatically exponentiate to an action of $G$. The brane $\mathcal{B}_{\mathrm{cc}}$ is $S L(2, \mathbb{C})$-invariant, since its ChanPaton curvature $\operatorname{Re} \Omega$ is invariant under $S L(2, \mathbb{C})$. A Lagrangian $A$-brane $\mathcal{B}^{\prime}$ will not be $S L(2, \mathbb{C})$-invariant. If $\mathcal{B}^{\prime}$ is invariant under a subgroup $G$ of $S L(2, \mathbb{C})$, then $G$ acts naturally on the space of $\left(\mathcal{B}_{\mathrm{cc}}, \mathcal{B}^{\prime}\right)$ strings. We use this starting in Sections 3.2 and 3.3 to construct representations of $S U(2)$ and $S L(2, \mathbb{R})$.

Actually, something less than $G$-invariance of $\mathcal{B}^{\prime}$ suffices to get an action of $G$ on the space of $\left(\mathcal{B}_{\mathrm{cc}}, \mathcal{B}^{\prime}\right)$ strings. What we need is only that, for all $g \in G$, the brane $\left(\mathcal{B}^{\prime}\right)^{g}$ obtained by transforming $\mathcal{B}^{\prime}$ by $g$ should be equivalent to $\mathcal{B}^{\prime}$ in the $A$-model. Hamiltonian isotopies of $Y$ with compact support, and more generally those that rapidly approach the identity at infinity, act trivially in the $A$-model. So if the $h$ action on $\mathcal{B}^{\prime}$ can be compensated by a Hamiltonian isotopy that has compact support, or that approaches the identity fast enough at infinity, then $\left(\mathcal{B}^{\prime}\right)^{g}$ is equivalent to $\mathcal{B}^{\prime}$ as an $A$-brane. If this holds for all $g \in G$, then $G$ acts on the space of $\left(\mathcal{B}_{\mathrm{cc}}, \mathcal{B}^{\prime}\right)$ strings. For examples, see Sections 3.7-8.

The functions $x, y$, and $z$ transform in the adjoint representation of $S O$ $(3, \mathbb{C})$. The polynomial functions in $x, y$, and $z$ modulo the commutation relations generate the universal enveloping algebra $\mathcal{U}$ of $S O(3, \mathbb{C})$. The ring $\mathcal{A}$ is a quotient $\mathcal{U} / \mathcal{I}$, where $\mathcal{I}$ is the ideal generated by $x^{2}+y^{2}+z^{2}-$ $\left(\mu^{2}-1\right) / 4$.

Now let us discuss hermitian structures. As explained in Section 2.4, to get a hermitian structure from branes, we need an antiholomorphic involution $\tau: Y \rightarrow Y$ that maps $\Omega$ to $\bar{\Omega}$. There are essentially two choices. The obvious choice

$$
\tau:(x, y, z) \rightarrow(\bar{x}, \bar{y}, \bar{z})
$$

has all the necessary properties and breaks $S O(3, \mathbb{C})$ to the compact subgroup $S O(3)$. Quantization of a $\tau$-invariant brane will therefore lead to unitary representations of $S O(3)$, or its double cover $S U(2)$. The alternative, 
up to a change of coordinates, is

$$
\tilde{\tau}:(x, y, z) \rightarrow(-\bar{x},-\bar{y}, \bar{z}) .
$$

This breaks $S O(3, \mathbb{C})$ to $S O(1,2)$, whose double cover is $S L(2, \mathbb{R})$. Quantization of $\tilde{\tau}$-invariant branes will lead to unitary representations of $S L(2, \mathbb{R})$.

\section{Parameters of the A-model}

A nice property of this model is that $Y$ actually admits a hyper-Kahler metric, extending the complex symplectic structure $(I, \Omega)$. In fact, with such a hyper-Kahler structure, $Y$ is known as the Eguchi-Hansen manifold. We write $I, J, K$ for the three complex structures and $\omega_{I}, \omega_{J}, \omega_{K}$ for the corresponding Kahler forms. The hyper-Kahler metric on $Y$ is completely determined by the three periods

$$
\begin{aligned}
& \int_{S} \frac{\omega_{I}}{2 \pi}=\alpha \\
& \int_{S} \frac{\omega_{J}}{2 \pi}=\beta \\
& \int_{S} \frac{\omega_{K}}{2 \pi}=\gamma
\end{aligned}
$$

where $\alpha, \beta, \gamma$ are arbitrary real parameters. These parameters are uniquely determined up to an overall sign $(\alpha, \beta, \gamma) \rightarrow(-\alpha,-\beta,-\gamma)$, which is equivalent to a reversal of orientation of $S$.

The holomorphic two-form in complex structure $I$ is $\Omega=\omega_{J}+i \omega_{K}$, so looking back to $(3.2)$, we see that the parameters are related by

$$
\mu=\beta+i \gamma
$$

(As before, $\mu= \pm \sqrt{\mu^{2}}$; the sign depends on the orientation of $S$, which also affects the sign of $\beta$ and $\gamma$.)

At the fixed point $\alpha=\beta=\gamma=0, Y$ develops an $A_{1}$ singularity. In fact, according to (3.11), at $\alpha=\beta=\gamma=0$, the equation for $Y$ reduces to $x^{2}+$ $y^{2}+z^{2}=0$, with a singularity at the origin. From the point of view of complex structure $I$, turning on $\alpha$ resolves the singularity, and turning on $\beta+i \gamma$ deforms it. 
The supersymmetric sigma-model with target $Y$ has a fourth parameter, a mode of the sigma-model $B$-field. This parameter is

$$
\eta=\int_{S} \frac{B}{2 \pi}
$$

and takes values in $\mathbb{R} / \mathbb{Z}$. A sign change of $\alpha, \beta, \gamma$ must be accompanied by a sign change of $\eta$, since it involves a reversal of orientation of $S$. The symmetry is therefore

$$
(\alpha, \beta, \gamma, \eta) \rightarrow(-\alpha,-\beta,-\gamma,-\eta)
$$

The parameter space of the model is therefore $\mathcal{W}=\left(\mathbb{R}^{3} \times S^{1}\right) / \mathbb{Z}_{2}$.

The present paper is really based on the $A$-model for symplectic structure $\omega_{K}$. This $A$-model is independent of $\alpha$ and $\beta$ (which control complex structure $K$ rather than symplectic structure $\omega_{K}$ ) and depends holomorphically on

$$
\lambda=\eta+i \gamma
$$

Indeed, $\gamma$ is a period of $\omega_{K}$, according to equation (3.10). As usual, the $A$-model parameters are obtained by complexifying the periods of the symplectic form to include $B$-field periods.

Though the $A$-model is independent of $\alpha$ and $\beta$, some $A$-branes are conveniently defined and studied for favorable values of those parameters. A case in point is the problem of defining a space-filling $A$-brane $\mathcal{B}_{\text {cc }}$ of rank 1. The Chan-Paton bundle of such a brane has a curvature two-form $F$ such that $\left(\omega_{K}^{-1}(F+B)\right)^{2}=-1$. (In discussing this equation in Section 2.1, we assumed that $B=0$.) A convenient way to solve this equation is to pick $\alpha$ and $\beta$, so as to determine a hyper-Kahler metric, and then require that

$$
F+B=\omega_{J}
$$

Dividing by $2 \pi$ and integrating over $S$, this implies that

$$
\int_{S} c_{1}(\mathcal{L})+\eta=\beta .
$$

Thus, to define a coisotropic brane in this way, we must pick $\beta$ so that $\beta-\eta$ is equal to the integer $n=\int_{S} c_{1}(\mathcal{L})$, which was already introduced in equation (3.3). For any given $A$-model parameter $\eta$, this construction can be made for any $n$, with $\beta$ adjusted accordingly. So the same $A$-model has 
a family of coisotropic branes depending on the integer $n$, though it is hard to use a single hyper-Kahler metric to construct all of them.

Comparing (3.16) with (3.11) and (3.14), we see that for a convenient choice of hyper-Kahler metric, the geometrical parameter $\mu^{2}$ is related to the $A$-model parameter $\lambda$ by

$$
\mu=\lambda+n
$$

In most of our discussion, we will set $\eta=0$, and we will pick a particular coisotropic brane with $n$ fixed. This means that we set $\beta=n$.

Now let us determine how the involutions $\tau$ and $\tilde{\tau}$, which are antiholomorphic in complex structure $I$, act on the parameters. Of course, neither $\tau$ nor $\tilde{\tau}$ is a symmetry unless $\mu^{2}$ is real. Thus, either $\gamma=0$ and $\mu^{2}=\beta^{2}$ is non-negative, or $\beta=0$ and $\mu^{2}=-\gamma^{2}$ is nonpositive.

In general, an antiholomorphic symmetry in complex structure $I$ reverses the sign of the Kahler form $\omega_{I}$. Also, $\tau$ and $\tilde{\tau}$ map $\Omega=\omega_{J}+i \omega_{K}$ to $\bar{\Omega}$, so they leave fixed $\omega_{J}$ and reverse the sign of $\omega_{K}$. To be symmetries of the sigma-model, $\tau$ and $\tilde{\tau}$ (which are not supposed to be orientifold symmetries; they should preserve the orientation of a string worldsheet) must preserve the $B$-field. The action of $\tau$ or $\tilde{\tau}$ on the parameters $(\alpha, \beta, \gamma, \eta)$ is therefore

$$
(\alpha, \beta, \gamma, \eta) \rightarrow \pm(-\alpha, \beta,-\gamma, \eta) .
$$

(The sign depends on whether $\tau$ or $\tilde{\tau}$ preserve the orientation of $S$, which as we see later can depend on the values of the parameters.) Thus, if $\beta \neq 0$, we may have $\eta \neq 0$ without spoiling $\tau$ or $\tilde{\tau}$ symmetry, but we must set $\alpha=\gamma=0$. And if $\gamma \neq 0$, we may have $\alpha \neq 0$, but we must set $\beta=\eta=0$.

\section{$3.2 \quad$ Representations of $S U(2)$}

We begin by considering unitary representations. To extract from the $A$-model a space of strings with a Hilbert space structure, we need to pick a second $A$-brane $\mathcal{B}^{\prime}$ that is supported at the fixed point set of $\tau$ or $\tilde{\tau}$.

We first consider the case of a brane $\mathcal{B}^{\prime}$ supported at the fixed point set of $\tau$. This fixed point set, which we will call $M$, consists of real $x, y, z$, obeying $x^{2}+y^{2}+z^{2}=\mu^{2} / 4$. In particular, $\mu^{2}=(\beta+i \gamma)^{2}$ must be real and positive (or $M$ is empty or collapses to a point, which is not a Lagrangian submanifold), so $\beta \neq 0, \gamma=0$. $M$ is $S U(2)$-invariant, so quantization of $\left(\mathcal{B}_{\mathrm{cc}}, \mathcal{B}^{\prime}\right)$ strings will lead to unitary representations of $S U(2)$. 
A Lagrangian brane supported on $M$ exists only if $\eta=0$. Indeed, if $F^{\prime}$ denotes the curvature of the Chan-Paton bundle of $\mathcal{B}^{\prime}$, then the Lagrangian condition is $\left.\left(F^{\prime}+B\right)\right|_{M}=0$. Given (3.12) (and the fact that $M$ coincides with $S$ in this example), this implies that $\int_{M} B / 2 \pi=0 \bmod \mathbb{Z}$, or $\eta=0$. Since also $\gamma=0,(3.17)$ tells us that

$$
\mu=n,
$$

where

$$
n=\int_{M} c_{1}(\mathcal{L})
$$

As usual $\mathcal{L}$ is the Chan-Paton bundle of $\mathcal{B}_{\text {cc }}$.

As $M$ is simply connected, the Chan-Paton bundle of $\mathcal{B}^{\prime}$ is trivial, so the space of $\left(\mathcal{B}_{\mathrm{cc}}, \mathcal{B}^{\prime}\right)$ strings is related to quantization of $M$ with prequantum line bundle $\left.\mathcal{L}\right|_{M}$.

An interpretation via quantization only exists if $\omega_{J}$ is nondegenerate when restricted to $M$. This implies that $n$ must be nonzero. We may as well orient $M$ so that $n>0$.

Quantization of $M$ with $n$ units of flux gives a Hilbert space $\mathcal{H}$ of dimension $n$, furnishing an irreducible representation of $S U(2)$ with $j=(n-1) / 2$. The quadratic Casimir operator $J^{2}=j(j+1)$ thus equals $\left(n^{2}-1\right) / 4$. Since $n^{2}=\mu^{2}$ according to equation (3.19), this is equivalent to $J^{2}=\left(\mu^{2}-1\right) / 4$. This result agrees with (3.7), and accounts for the choice of constant in this formula.

The generators $J_{x}, J_{y}$, and $J_{z}$ of $S U(2)$ arise by quantizing the functions $x, y$, and $z$. We diagonalize $J_{z}$, finding a basis of eigenstates of $J_{z}$, each obeying $J_{z} \psi_{s}=s \psi_{s}$ for some $s$. According to a standard analysis, in an $n$-dimensional irreducible representation of $S U(2)$, the values of $s$ are

$$
-\frac{n-1}{2}, 1-\frac{n-1}{2}, 2-\frac{n-1}{2}, \ldots, \frac{n-1}{2} .
$$

The range of eigenvalues of $J_{z}$ is in accord with the classical fact that on the brane characterized by $x^{2}+y^{2}+z^{2}=n^{2} / 4$ (with real $x, y, z$ ) $z$ is bounded by $-n / 2 \leq z \leq n / 2$. Because of quantum mechanical fluctuations, the maximal value of $\left|J_{z}\right|$ in the quantum theory (namely $(n-1) / 2$ ) is slightly less than the classical upper bound $n / 2$. 


\subsection{Discrete series of $S L(2, \mathbb{R})$}

To construct unitary representations of $S L(2, \mathbb{R})$, we proceed in a similar way, but now we consider $\tilde{\tau}$-invariant branes. It is convenient to make a change of coordinates $x \rightarrow i x, y \rightarrow i y$, so that the equation defining $Y$ becomes

$$
-x^{2}-y^{2}+z^{2}=\frac{\mu^{2}}{4}
$$

and $\tilde{\tau}$ acts simply by

$$
(x, y, z) \rightarrow(\bar{x}, \bar{y}, \bar{z})
$$

We continue to assume that $\eta=0$, and to ensure that $Y$ admits the symmetry (3.23), we take $\mu^{2}$ real. We begin with the case $\mu^{2}>0$, which means that $\gamma=0, \beta \neq 0$. Just as in the last subsection, existence of the brane $\mathcal{B}_{\text {cc }}$ requires us to take $\beta$ to be an integer. This integer is $n=\int_{S} c_{1}(\mathcal{L})$, where now $S \subset Y$ is given by imaginary $x$ and $y$ and real $z$. As in Section 3.2 , we may as well take $n \geq 0$. However, in Section 3.2, we required $n>0$ to ensure that $\omega_{J}$ remains nondegenerate when restricted to $S$. For our present purposes, this is irrelevant (we will not consider a brane wrapped on $S$ ), so we allow $n=0$. For $n=0$, the equation defining $Y$ reduces to $-x^{2}-y^{2}+z^{2}=0$, with an $A_{1}$ singularity at $z=y=z=0$, so we should expect that some unusual behavior may occur at that point.

The fixed point set of $\tilde{\tau}$ consists of real $x, y, z$. The equation $z^{2}=\mu^{2} / 4+$ $x^{2}+y^{2}$ shows that this fixed point set has two components $M_{ \pm}$, given by

$$
z= \pm \sqrt{\mu^{2} / 4+x^{2}+y^{2}} .
$$

Each component is equivalent to the complex upper half-plane with the usual action of $S L(2, \mathbb{R})$. In particular, the components $M_{ \pm}$are simply connected, and each supports a unique rank $1 A$-brane $\mathcal{B}_{ \pm}^{\prime}$.

We quantize $M_{ \pm}$by taking the space of $\left(\mathcal{B}_{\mathrm{cc}}, \mathcal{B}_{ \pm}^{\prime}\right)$ strings. This should give a unitary representation $D_{n}^{ \pm}$of $S L(2, \mathbb{R})$ with the same value of the quadratic Casimir that we found in Section 3.2, namely $J^{2}=\left(n^{2}-1\right) / 4$. (The value of the quadratic Casimir must be the same because it is determined entirely by the choice of the space $Y$ and the brane $\mathcal{B}_{\mathrm{cc}}$, independent of the choice of a second $A$-brane $\mathcal{B}^{\prime}$.) On the classical phase space $M_{ \pm}$, the range of values of $z$ is $n / 2 \leq z \leq \infty$ on $M_{+}$and $-\infty \leq z \leq-n / 2$ on $M_{-}$. Hence in the representation $D_{n}^{+}, J_{z}$ should be unbounded above, but bounded below by 
approximately $n / 2$; and in the representation $D_{n}^{-}, J_{z}$ should be unbounded below, but bounded above by approximately $-n / 2$.

Unitary representations of $S L(2, \mathbb{R})$ with these properties do exist and are known as the discrete series. The precise set of values of $J_{z}$ is

$$
\frac{n+1}{2}, \quad 1+\frac{n+1}{2}, \quad 2+\frac{n+1}{2}, \ldots
$$

for $D_{n}^{+}$and

$$
-\frac{n+1}{2}, \quad-1-\frac{n+1}{2}, \quad-2-\frac{n+1}{2}, \ldots
$$

for $D_{n}^{-}$. We will explain these formulas in Section 3.5 after describing the principal series.

\subsection{Principal series of $S L(2, \mathbb{R})$}

Now we consider the case $\mu^{2}<0$, that is $\beta=0, \gamma \neq 0$. The fixed point set $M$ of $\tilde{\tau}$ is now given by the equation

$$
x^{2}+y^{2}=\frac{\gamma^{2}}{4}+z^{2},
$$

with $x, y$, and $z$ real. It is connected, with the topology of $\mathbb{R} \times S^{1}$ (for $\gamma \neq 0)$. $z$ is unbounded above and below, so in a representation obtained by quantizing $M, J_{z}$ is similarly unbounded.

The first Betti number of $M$ is 1 , so there is a one-parameter family $\mathcal{B}_{\delta}^{\prime}$ of rank $1 A$-branes supported on $M$; roughly speaking, the monodromy of the Chan-Paton bundle of $\mathcal{B}_{\delta}^{\prime}$ around the circle in $M$ is $\exp (2 \pi \mathrm{i} \delta)$. To be more precise, we recall that the Chan-Paton bundle of an $A$-brane is actually a flat $\operatorname{Spin}_{\mathrm{c}}$ bundle (not a flat line bundle), so we actually need to pick a spin structure in order to define $\delta$ as a number. To define $\delta$ precisely, we declare that $\delta=0$ corresponds to the Ramond spin structure (the one that corresponds to a trivial real line bundle over a circle).

Topologically, $\mathbb{R} \times S^{1}$ is the same as the cotangent bundle $T^{*} S^{1}$. We explain below how to make contact between the present problem and quantization of $M=T^{*} S^{1}$ with its standard symplectic structure. For now, we just proceed informally. In quantizing $M=T^{*} S^{1}$ via functions (actually halfdensities) on $S^{1}$, a wavefunction picks up a phase $\exp (2 \pi \mathrm{i} \delta)$ in going around 
$S^{1}$. As a result, the eigenvectors of $J_{z}$ are exponentials $\exp (\mathrm{i}(n+\delta) \theta)$, where $\theta$ is an angular variable on $S^{1}$, and $n$ is any integer. The spectrum of $J_{z}$ is thus of the form $\{\delta+n \mid n \in \mathbb{Z}\}$, and is unbounded above and below, as expected.

To get a representation of $S L(2, \mathbb{R})$, the eigenvalues of $J_{z}$ must be integers or half-integers, and so $\delta$ must be 0 or $1 / 2$. For generic $\delta$, we get a representation not of $S L(2, \mathbb{R})$, but of its universal cover, which we denote $\widetilde{S L}(2, \mathbb{R})$.

The representations obtained from this construction are known as the principal series representations $P_{\gamma, \delta}$. These are representations of $S L(2, \mathbb{R})$ (or its universal cover) with quadratic Casimir

$$
J^{2}=-\frac{\gamma^{2}+1}{4}
$$

for real $\gamma$. The spectrum of $J_{z}$ is $\{\delta+n \mid n \in \mathbb{Z}\}$, as we described above, and we get a representation of $S L(2, \mathbb{R})$ (as opposed to a cover) precisely if $\delta=0$ or $1 / 2$. The central element $\operatorname{diag}(-1,-1)$ of $S L(2, \mathbb{R})$, which corresponds to a $2 \pi$ rotation of the circle, acts by $\exp (2 \pi \mathrm{i} \delta)$.

A standard algebraic analysis (which we will essentially explain in Section 3.5) shows that $P_{\gamma, \delta}$ is irreducible unless $\gamma=0$ and $\delta=1 / 2$. In that case, one gets

$$
P_{0, \frac{1}{2}}=D_{0}^{+} \oplus D_{0}^{-}
$$

where $D_{0}^{ \pm}$is the $n=0$ case of the discrete series representations $D_{n}^{ \pm}$of Subsection 3.3. Let us try to understand this decomposition from the present point of view.

If we set $\gamma=0, M$ reduces to the cone $z^{2}=x^{2}+y^{2}$. It is a union of two components with $z \geq 0$ and $z \leq 0$, respectively. These are the specialization to $n=0$ of the two symplectic manifolds $M_{+}$and $M_{-}$whose quantization led to the discrete series. Topologically, each of $M_{+}$and $M_{-}$is a disc, with a unique flat $\operatorname{Spin}_{\mathrm{c}}$ structure that actually is a spin structure (since $M_{ \pm}$are simply connected). On a circle at infinity, this spin structure corresponds to the bounding or Neveu-Schwarz spin structure, which we get at $\delta=1 / 2$. So it is only at $\delta=1 / 2$ that a brane supported on $M$ decomposes (when we set $\gamma$ to zero) as the sum of a brane supported on $M_{+}$and one supported on $M_{-}$. At any other value of $\delta, M_{+}$and $M_{-}$are linked by a monodromy at the origin. 
The singularity at $\gamma=0$

At $\gamma=0, Y$ is described by $x^{2}+y^{2}-z^{2}=0$, with an $A_{1}$ singularity at the origin. To understand better this case, we exploit the fact that the parameter $\alpha$ is trivial in the $A$-model. We can turn on $\alpha$ and remove the singularity without changing the $A$-model. Moreover, since we here have $\beta=0$, this does not disturb $\tilde{\tau}$ symmetry.

Turning on $\alpha$ has the effect of blowing up the singularity. A convenient way to describe the blowup is as follows. Consider a space $\mathbb{C}^{3}$, with coordinates $(a, b, p)$, subject to a scaling symmetry $(a, b, p) \rightarrow\left(t a, t b, t^{-2} p\right), t \in \mathbb{C}^{*}$. The ring of $\mathbb{C}^{*}$-invariant functions is generated by

$$
\begin{aligned}
x+z & =a^{2} p \\
x-z & =-b^{2} p, \\
y & =a b p,
\end{aligned}
$$

subject to one relation $x^{2}+y^{2}-z^{2}=0$. Let $Z$ be the locus in $\mathbb{C}^{3}$ consisting of points in which $a$ and $b$ are not both zero. The quotient $Y^{\prime}=Z / \mathbb{C}^{*}$ has a natural map to $Y$, given by (3.30). This map is one-to-one except that the inverse image of the origin in $Y$ is a copy of $\mathbb{C P}^{1}$ given by $p=0$. Thus, $Y^{\prime}$ is obtained (in complex structure $I$ ) by blowing up the singularity of $Y$ at the origin. The holomorphic two-form of $Y^{\prime}$, which corresponds to $\Omega$ under the above map, is $\Omega^{\prime}=(a d b-b d a) \wedge d p-2 p d a \wedge d b$.

The Lagrangian submanifold $M^{\prime}$ that corresponds to $M$ is defined by taking $a, b, p$ real. To describe $M^{\prime}$ explicitly, we use the scaling symmetry to set $a^{2}+b^{2}=1$. This leaves a freedom of reversing the sign of $a$ and $b$, so we set $a=\cos (\theta / 2), b=\sin (\theta / 2)$ for some angle $\theta$. The symplectic structure with which we need to quantize $M^{\prime}$ is $\omega_{J}^{\prime}=\operatorname{Re} \Omega^{\prime}=d p \wedge$ $d \theta$. This is the standard symplectic structure of $T^{*} S^{1}$. Moreover, the action of $S L(2, \mathbb{R})$ on $T^{*} S^{1}$ is the natural action coming from the usual action of $S L(2, \mathbb{R})$ on $S^{1} \cong \mathbb{R}^{1}$. Quantization leads to half-densities on $S^{1}$, with the natural action of $S L(2, \mathbb{R})$ on the space of half-densities. If we take $\delta \neq 0$, we get half-densities twisted by a flat bundle of monodromy $\exp (2 \pi \mathrm{i} \delta)$. This leads to the spectrum of $J_{z}$ described above. (In this description, the reducibility of the $S L(2, \mathbb{R})$ representation at $\delta=1 / 2$ is not clear.)

When $\gamma \neq 0, M$ is smooth and the blowup via $\alpha$ is not necessary. In this case, by setting $(x, y, z)=\frac{1}{2} \gamma(\cosh p \cos \theta, \cosh p \sin \theta, \sinh p)$, we can identify $M$ with $T^{*} S^{1}$ with its usual symplectic structure. This identification commutes with the group of rotations of the circle, so it can be used to 
determine the spectrum of $J_{z}$, but it does not commute with $S L(2, \mathbb{R})$, so it does not directly determine the $S L(2, \mathbb{R})$ representation. It can be shown that the representation $P_{\gamma, \delta}$ can be interpreted as the space of densities of weight $1 / 2+\mathrm{i} \gamma$ twisted by a flat line bundle with monodromy $\exp (2 \pi \mathrm{i} \delta)$, with the natural action of $\widetilde{S L}(2, \mathbb{R})$ on this space. One way to show this is to use the fact that $M$ has an $S L(2, \mathbb{R})$-invariant map to $S^{1}$ with Lagrangian fibers; in other words, $M$ admits an $S L(2, \mathbb{R})$-invariant real polarization. (In fact, $M$ has two such polarizations.)

\subsection{Algebraic description}

To better understand some things that we have already encountered and in preparation for what follows, we will summarize some standard facts about representations of $\mathfrak{s l}(2)$ from an algebraic point of view.

In our construction, quantization of the functions $x, y$, and $z$ gives operators $J_{x}, J_{y}, J_{z}$ that obey the usual $\mathfrak{s l}(2)$ commutation relations such as $\left[J_{x}, J_{y}\right]=\mathrm{i} J_{z}$. As usual, it is convenient to set $J_{ \pm}=J_{x} \pm \mathrm{i} J_{y}$. For unitary representations of $\mathfrak{s u}(2), J_{z}$ is hermitian and $J_{+}$is the hermitian adjoint of $J_{-}$. For unitary representations of $\mathfrak{s l}(2, \mathbb{R}), J_{z}$ is hermitian and $J_{+}$is minus the adjoint of $J_{-}$. We summarize the facts about $J_{ \pm}$:

$$
J_{+}^{\dagger}= \begin{cases}J_{-} & \text {for } \mathfrak{s u}(2) \\ -J_{-} & \text {for } \mathfrak{s l}(2, \mathbb{R}) .\end{cases}
$$

We will consider both unitary representations and representations that are not required to be unitary. In the unitary case, since $J_{z}$ is hermitian, it can be diagonalized with real eigenvalues, but in any event, we only consider representations in which $J_{z}$ can be diagonalized. We also assume that the quadratic Casimir operator can be diagonalized, and we often write simply $J^{2}$ for its eigenvalue.

Suppose that $\psi$ is an eigenvector of $J_{z}$, with $J_{z} \psi=s \psi$ for some $s$. Additional eigenvectors can be constructed by acting with $J_{+}$or $J_{-}$. This process may continue indefinitely in both directions, giving a representation in which $J_{z}$ (or more exactly the real part of its eigenvalue) is unbounded above and below. Alternatively, it may terminate in either or both directions, if we find a highest weight vector $\psi$ obeying

$$
J_{z} \psi=s_{+} \psi, \quad J_{+} \psi=0,
$$


or a lowest weight vector $\tilde{\psi}$ obeying

$$
J_{z} \tilde{\psi}=s_{-} \tilde{\psi}, \quad J_{-} \tilde{\psi}=0 .
$$

In an irreducible representation, the quadratic Casimir operator $J^{2}=$ $J_{x}^{2}+J_{y}^{2}+J_{z}^{2}$ is a multiple of the identity. It can be written as

$$
\begin{aligned}
J^{2} & =J_{z}^{2}+J_{z}+J_{-} J_{+} \\
& =J_{z}^{2}-J_{z}+J_{+} J_{-} .
\end{aligned}
$$

If (3.32) is obeyed, we use the first formula in (3.34) to deduce that

$$
J^{2}=s_{+}\left(s_{+}+1\right),
$$

and if (3.33) is obeyed, we use the second formula to deduce that

$$
J^{2}=s_{-}\left(s_{-}-1\right) \text {. }
$$

If (3.35) is obeyed for some value of $s_{+}$, then (3.36) is obeyed with

$$
s_{-}=s_{+}+1 \text {. }
$$

Thus, it is possible to have a representation of highest weight $s_{+}$if and only if it is possible for the same value of the quadratic Casimir to have a representation of lowest weight $s_{+}+1$. This explains the relation between the bounds on $J_{z}$ for finite-dimensional representations in (3.21) and the corresponding bounds for discrete series representations in (3.25) and (3.26).

If we assume unitarity, we can learn a little more. A vector $\psi$ obeying $J_{z} \psi=s_{+} \psi$, where $s_{+}$obeys (3.35), must be annihilated by $J_{+}$. Indeed, we have $J_{-} J_{+} \psi=\left(J^{2}-J_{z}^{2}-J_{z}\right) \psi=0$. So $0=\left(\psi, J_{-} J_{+} \psi\right)$. In the unitary case, the right-hand side is $\pm\left(J_{+} \psi, J_{+} \psi\right)$, and its vanishing implies that $J_{+} \psi=0$. Similarly, if $J_{z} \tilde{\psi}=s_{-} \tilde{\psi}$, and $s_{-}$obeys (3.36), then in the unitary case, it follows that $J_{-} \tilde{\psi}=0$.

If $\psi$ is a highest weight vector with $J_{z} \psi=s_{+} \psi$, then $s_{+} \geq 0$ for a unitary representation of $\mathfrak{s u}(2)$, and $s_{+} \leq 0$ for a unitary representation of $\mathfrak{s l}(2, \mathbb{R})$. The two statements can be combined to $\epsilon s_{+} \geq 0$, where $\epsilon=1$ for $\mathfrak{s u}(2)$ and $\epsilon=-1$ for $\mathfrak{s l}(2, \mathbb{R})$. To see this, we note that $J_{+} \psi=0$ implies that $2 s_{+}(\psi, \psi)=\left(\psi, 2 J_{z} \psi\right)=\left(\psi,\left[J_{+}, J_{-}\right] \psi\right)=\left(\psi, J_{+} J_{-} \psi\right)=\epsilon\left(J_{-} \psi, J_{-} \psi\right)$, where (3.31) was used in the last step. This indeed implies that $\epsilon s_{+} \geq 0$, with equality only if $J_{-} \psi=0$, which implies that $\psi$ generates a one-dimensional trivial representation. A similar argument shows that a lowest weight vector 
has $s_{-} \leq 0$ in the case of a unitary representation of $\mathfrak{s u}(2)$, and $s_{-} \geq 0$ in the case of a unitary representation of $\mathfrak{s l}(2, \mathbb{R})$.

A standard fact, which we will not review here, is that every irreducible representation of $S U(2)$ has both a highest weight vector $\psi$ and a lowest weight vector $\tilde{\psi}$. Moreover, we can assume that $\tilde{\psi}=J_{-}^{n} \psi$ for some integer $n$. If $J_{z} \psi=s_{+} \psi$, then $J_{z} \tilde{\psi}=\left(s_{+}-n\right) \tilde{\psi}$. The quadratic Casimir is $J^{2}=$ $s_{+}\left(s_{+}+1\right)=\left(s_{+}-n\right)\left(s_{+}-n-1\right)$, implying that $s_{+}=n / 2$ is an integer or a half-integer.

For the case of a nontrivial irreducible unitary representation of $\mathfrak{s l}(2, \mathbb{R})$, it is impossible to have both a lowest weight vector and a highest weight vector. The $J_{z}$ eigenvalues would have to be negative for the highest weight vector, and positive for the lowest weight vector; but a highest weight vector has a higher weight than any other vector in an irreducible representation. This being so, we cannot make an argument like the one in the last paragraph, and there is no way to show algebraically that the eigenvalues of $J_{z}$ take values in $\mathbb{Z} / 2$. When the eigenvalues are valued in $\mathbb{Z} / 2$, we get a representation of the group $S L(2, \mathbb{R})$, while the more general case leads to representations of its universal cover. In Sections 3.3-4, we have encountered unitary representations of $S L(2, \mathbb{R})$ with neither a highest weight vector nor a lowest weight vector (the principal series), as well as unitary representations with a lowest weight vector (the discrete series $D_{n}^{+}$) or a highest weight vector $\left(D_{n}^{-}\right)$.

\section{Relaxing unitarity}

Now let us consider representations that are not necessarily unitary. The quadratic Casimir can act as a complex number, which we simply call $J^{2}$, and likewise the eigenvalues of $J_{z}$ can be complex. Consider a representation that contains a vector $\psi$ with $J_{z} \psi=s \psi$ for some $s$. If $s$ and $J^{2}$ are generic, then there does not exist an integer $n$ such that $J^{2}$ is equal to $(s+n)(s+n+1)$. In this case, none of the states $J_{+}^{n} \psi$ or $J_{-}^{m} \psi$ have eigenvalues obeying (3.35) or (3.36), so these states are all nonzero. So a representation with generic $s$ and $J^{2}$ is infinite in both directions, like the principal series representations.

For special values of $s$ and $J^{2}$, something special can happen. If $J^{2}=$ $s(s+1)$, it is possible to have a representation $R^{-}$spanned by vectors $\psi_{n}, n=0,-1,-2, \ldots$, with $J_{z} \psi_{n}=(s+n) \psi_{n}$. This representation has a highest weight vector $\psi_{0}$ with $J_{z} \psi_{0}=s \psi_{0}$. It is also possible to have a representation $R^{+}$spanned by vectors $\psi_{n}, \quad n=1,2,3 \ldots$, again with $J_{z} \psi_{n}=(s+n) \psi_{n}$. This representation has a lowest weight vector $\psi_{1}$ with $J_{z} \psi_{1}=(s+1) \psi_{1}$. 
Now let us consider a general representation $R$ spanned by vectors $\psi_{n}$, $n \in \mathbb{Z}$, obeying $J_{z} \psi_{n}=(s+n) \psi_{n}$. Suppose that $J_{+} \psi_{n}=a_{n} \psi_{n+1}, J_{-} \psi_{n+1}=$ $b_{n} \psi_{n}$, for some complex constants $a_{n}, b_{n}$. Again, write $J^{2}$ for the eigenvalue of the quadratic Casimir operator. Using (3.34), we deduce that for all $n$,

$$
a_{n} b_{n}=J^{2}-(s+n)(s+n+1) .
$$

This is a necessary and sufficient condition to get a representation of the Lie algebra $\mathfrak{s l}(2, \mathbb{C})$ with the assumed value of the Casimir. For every $n$, we are free to redefine $\psi_{n} \rightarrow \lambda_{n} \psi_{n}$, for $\lambda_{n} \in \mathbb{C}^{*}$, along with

$$
a_{n} \rightarrow \lambda_{n} \lambda_{n+1}^{-1} a_{n}, b_{n} \rightarrow \lambda_{n+1} \lambda_{n}^{-1} b_{n} .
$$

As long as $J^{2}$ and $s$ are such that $J^{2}-(s+n)(s+n+1)$ never vanishes, $a_{n}$ and $b_{n}$ are nonzero and are uniquely determined, modulo a transformation of the type (3.39), by the equations (3.38). This gives an irreducible representation for the assumed values of $J^{2}$ and $s$, generalizing the principal series.

Suppose on the other hand that $J^{2}-(s+n)(s+n+1)=0$ for some value of $n$. Then we have $a_{n} b_{n}=0$, leaving three choices:

(1) We may have $a_{n}=b_{n}=0$. This gives a representation $R$ that decomposes as a direct sum $R=R_{1} \oplus R_{2}$, where $R_{1}$ has a highest weight vector $\psi_{n}$ and $R_{2}$ has a lowest weight vector $\psi_{n+1}$.

(2) We may have $b_{n}=0, a_{n} \neq 0$. This gives a representation $R$ that contains a subrepresentation $R_{2}$ spanned by vectors $\psi_{m}, m \geq n+1 . R_{2}$ has a lowest weight vector $\psi_{n+1}$. There is no complementary representation $R_{1}$, but rather $R$ can be described as an extension:

$$
0 \rightarrow R_{2} \rightarrow R \rightarrow R_{1} \rightarrow 0 .
$$

Here $R_{1}$ is spanned by $\psi_{m}, m \leq n$, but is not a subrepresentation of $R$, since $a_{n} \neq 0$ and $\psi_{n}$ is not a highest weight vector. However, $R_{1}$ can be understood as a quotient representation $R / R_{2}$.

(3) Finally, we may have $a_{n}=0, b_{n} \neq 0$. This gives an extension in the opposite direction:

$$
0 \rightarrow R_{1} \rightarrow R \rightarrow R_{2} \rightarrow 0 .
$$

Here $R_{1}$ is the subrepresentation spanned by $\psi_{m}, m \leq n$, and containing the highest weight vector $\psi_{n} . R_{2}$ is the quotient representation $R / R_{1}$. 
The phenomenon just described involving nonsplit extensions does not occur for representations of either real form of $\mathfrak{s l}(2)$ that admit a nondegenerate hermitian form, since in that case $b_{n}= \pm \bar{a}_{n}$ in view of (3.31). What nonsplit extensions mean in terms of branes will be described in Section 3.7.

If we further specialize $s$ and $J^{2}$, the equation $J^{2}=(s+n)(s+n+1)$ may have two integer solutions, say $n_{1}$ and $n_{2}$. In this case, we run into the two equations $a_{n_{1}} b_{n_{1}}=0$ and $a_{n_{2}} b_{n_{2}}=0$, and we can independently choose, in each of the two cases, which if either of $a$ and $b$ is nonzero.

\section{Hermitian structure}

Now let us specialize this to the case of a representation of $\mathfrak{s l}(2, \mathbb{R})$ compatible with a hermitian structure ( , ). The relations $J_{+} \psi_{n}=a_{n} \psi_{n+1}$, $J_{-} \psi_{n+1}=b_{n} \psi_{n} \quad$ imply $\quad\left(\psi_{n+1}, J_{+} \psi_{n}\right)=a_{n}\left(\psi_{n+1}, \psi_{n+1}\right),\left(\psi_{n}, J_{-} \psi_{n+1}\right)=$ $b_{n}\left(\psi_{n}, \psi_{n}\right)$. If $J_{-}=-J_{+}^{\dagger}$, then $\left(\psi_{n}, J_{-} \psi_{n+1}\right)$ is minus the complex conjugate of $\left(\psi_{n+1}, J_{+} \psi_{n}\right)$, so we get

$$
a_{n} b_{n}=-\frac{\left|\left(\psi_{n+1}, J_{+} \psi_{n}\right)\right|^{2}}{\left(\psi_{n+1}, \psi_{n+1}\right)\left(\psi_{n}, \psi_{n}\right)} .
$$

For a unitary representation, $\left(\psi_{n}, \psi_{n}\right)>0$ for all $n$. In this case, (3.42) implies that $a_{n} b_{n}<0$ (unless $\psi_{n}$ is a highest weight vector). Let us apply this to a unitary representation in which the set of $J_{z}$ eigenvalues is $\{s+n \mid n \in \mathbb{Z}\}$ for some real $s$. Together with the fact that $a_{n} b_{n}<0,(3.38)$ implies that $(s+n)(s+n+1)>J^{2}$ for all $n$, or

$$
(s+n+1 / 2)^{2}>J^{2}+1 / 4 .
$$

For example, let us consider representations of $S L(2, \mathbb{R})$ in which $s=0$ so that the central element $\operatorname{diag}(-1,-1)$ acts trivially. The condition that $(n+1 / 2)^{2}>J^{2}+1 / 4$ for all integers $n$ is equivalent to $J^{2}<0$. We have already encountered the relevant unitary representations with $J^{2} \leq-1 / 4$. These are the principal series representations with $J^{2}=-\left(\gamma^{2}+1\right) / 4$. The unitary representations with $0>J^{2}>-1 / 4$ are known as the complementary series. Algebraically, the complementary series is simply the analytic continuation of the principal series to $J^{2}>-1 / 4$ or imaginary $\gamma$. (Equation (3.38) with the equivalence relation (3.39) give a general description for all $J^{2}$ and s.) However, natural geometric realizations of the hermitian structure undergo a sort of phase transition at $J^{2}=-1 / 4$. For an explanation of how this happens (in a standard description of the representations via densities of suitable weight on $S^{1}$ ), see chapter 1.3 of [26]. In terms of 
branes, we described the principal series in Section 3.4, and we will describe the complementary series in Section 3.8.

A representation of $\mathfrak{s l}(2, \mathbb{R})$ with any real $J^{2}$ and $s$ can admit a nondegenerate hermitian structure, though not necessarily a positive-definite one. Let us see what happens for $s=0$ and $J^{2}>-1 / 4$. If there is an integer $n$ such that $J^{2}=n(n+1)$, the representation has a lowest weight or highest weight vector, as we have seen above. Let us suppose that this is not the case. Equations (3.38) and (3.42) imply that

$$
\left(\psi_{n}, \psi_{n}\right)\left(\psi_{n+1}, \psi_{n+1}\right)=\left(n(n+1)-J^{2}\right)\left|\left(\psi_{n+1}, J_{+} \psi_{n}\right)\right|^{2} .
$$

Let $n_{0}$ be the largest integer such that $n_{0}\left(n_{0}+1\right)-J^{2}<0$. Then the condition $n(n+1)-J^{2}<0$ is obeyed for $2 n_{0}+2$ values of $n$, namely $-n_{0}-1 \leq$ $n \leq n_{0}$. From (3.44), it follows that if $n$ is in this range, then $\left(\psi_{n+1}, \psi_{n+1}\right)$ and $\left(\psi_{n}, \psi_{n}\right)$ have opposite signs. The total number of sign changes is even, so if $\left(\psi_{n}, \psi_{n}\right)$ is positive for large positive $n$, then it is also positive for large negative $n$. However, the $n_{0}+1$ states $\psi_{n_{0}-2 k}, 0 \leq k \leq n_{0}$, have negative norm.

A similar analysis can be made for other values of $s$. For brevity, we consider only the question of $s=1 / 2$, which is associated to representations of $S L(2, \mathbb{R})$ in which the central element acts as -1 . For $s=1 / 2$, (3.43) tells us that $-1 / 4 \geq J^{2}$, which is the range covered by the principal series. So there are no new unitary representations to be had. There are, of course, representations with indefinite but nondegenerate hermitian forms. For $J^{2}>-1 / 4$ and $s=1 / 2$, the number of sign changes is odd, so if $\left(\psi_{n}, \psi_{n}\right)$ is positive for large positive $n$, it is negative for large negative $n$. In the region near $n=0$, the signs alternate.

\subsection{Discrete series of $\widetilde{S L}(2, \mathbb{R})$}

It is now straightforward to complete the description of the discrete series. For a unitary representation, $\mu^{2}=(\beta+i \gamma)^{2}$ must be real, so either $\beta$ or $\gamma$ vanishes. We have already considered in Section 3.4 the case of $\beta=0$ with generic $\gamma$. So here we take $\gamma=0$ with generic $\beta$.

From (3.16), we have $\beta=\eta+n$, where $n=\int_{S} c_{1}(\mathcal{L})$ is an integer and $\eta$ is the world-sheet theta-angle. Thus, to get generic $\beta$, we must take $\eta$ to be nonzero. The quadratic Casimir is $J^{2}=\left(\beta^{2}-1\right) / 4$, just as in the case of integer $\beta$. As in Section 3.2, we introduce a second $A$-brane $\mathcal{B}_{ \pm}^{\prime}$ supported on the locus $M_{ \pm}$defined by $z= \pm \sqrt{\mu^{2}+x^{2}+y^{2}}$. (The Chan-Paton bundle of this brane is determined up to isomorphism by the requirement that 
$F+\left.B\right|_{M}=0$.) Classically, on the support of this brane, $z$ is bounded below or above by $\mu$.

Quantum mechanically, there must be a lowest weight or highest weight vector. The $J_{z}$ eigenvalue $s_{-}$of a lowest weight vector is determined by $s_{-}\left(s_{-}-1\right)=J^{2}=\left(\beta^{2}-1\right) / 4$ or

$$
s_{-}=\frac{\beta+1}{2} .
$$

The spectrum is exactly as in (3.25), with $n$ replaced by $\beta$. For nonintegral $n, J_{z}$ is no longer a half-integer. What we get this way is a unitary representation of the universal cover $\widetilde{S L}(2, \mathbb{R})$ of $S L(2, \mathbb{R})$. It is the generalization of the discrete series to $\widetilde{S L}(2, \mathbb{R})$. See $[27]$ for more.

\subsection{Harish-Chandra modules from branes}

Our next goal is to describe in terms of branes the not necessarily unitary representations that were described algebraically in Section 3.5, for example in equations (3.38) and (3.39). These are representations in which the spectrum of $J_{z}$ is of the form $s+n$, for some complex constant $s$ and for $n$ ranging over a sequence of consecutive integers that may be infinite, semi-infinite, or finite. Such representations are known mathematically as Harish-Chandra modules. (Sometimes one considers only the case $2 s \in \mathbb{Z}$, corresponding to representations of $S L(2, \mathbb{R})$ rather than a cover, but we will not make this restriction.)

In general, Harish-Chandra modules are not unitary, so we will have to describe them via branes that are not $\tilde{\tau}$-invariant. Harish-Chandra modules do admit an action of the group $S L(2, \mathbb{R})$, though in general not a unitary action. This suggests that we might describe them via $S L(2, \mathbb{R})$-invariant branes, but in fact, the full list of $S L(2, \mathbb{R})$-invariant Lagrangian submanifolds of $Y$ is very short and we have exhausted it already. However, since sufficiently well localized Hamiltonian isotopies act trivially in the $A$-model, it suffices for our purposes to consider branes that are $S L(2, \mathbb{R})$-invariant only asymptotically. It turns out that there is a sufficient supply of these.

Since $J_{z}$ can be naturally diagonalized in these modules, it is reasonable to guess that one can choose the brane $\mathcal{B}^{\prime}$ to be invariant under the subgroup $\mathrm{K} \cong U(1)=S O(2)$ generated by $J_{z} . \mathrm{K}$ is a maximal compact subgroup of $S L(2, \mathbb{R})$. 
As we will see, it is possible to describe all K-invariant Lagrangian submanifolds of $Y$. It is convenient to use the fact that $Y$ admits a family of hyper-Kahler metrics with parameters $(\alpha, \beta, \gamma)$, as described in Section 3.1. The hyper-Kahler metric is not $S L(2, \mathbb{C})$-invariant, but it is invariant under the maximal compact subgroup $S U(2) \subset S L(2, \mathbb{C})$ and in particular under the maximal compact subgroup $\mathrm{K}=U(1) \subset S L(2, \mathbb{R})$. The moment map for the $\mathrm{K}$ action gives three natural functions on $Y$, which we denote as $\vec{w}$. (Thus the components of $\vec{w}$ are the moment maps for $\mathrm{K}$ with respect to the three symplectic structures $\vec{\omega}=\left(\omega_{I}, \omega_{J}, \omega_{K}\right)$.) Mapping a point in $Y$ to the corresponding value of $\vec{w}$ gives a map $\pi: Y \rightarrow \mathbb{R}^{3}$, which away from the fixed points of $\mathrm{K}$ is a fibration with $S^{1}$ fibers. The hyper-Kahler metric of $Y$ takes the form

$$
d s^{2}=H d \vec{w} \cdot d \vec{w}+H^{-1}(d \chi+\vec{a})^{2}
$$

where $\chi \cong \chi+2 \pi$ is an angular parameter along the fibers of $\pi$. The group $\mathrm{K}$ acts by translation of $\chi$ or in other words by rotation of the fibers. $H$ is the harmonic function

$$
H=\frac{1}{2\left|\vec{w}-\vec{w}^{*}\right|}+\frac{1}{2\left|\vec{w}+\vec{w}^{*}\right|} .
$$

The moduli of the hyper-Kahler metric are contained in the choice of point $\vec{w}^{*} \in \mathbb{R}^{3}$ :

$$
\vec{w}^{*}=\frac{1}{2}(\alpha, \beta, \gamma)
$$

This formula will be explained shortly. At the two points $\vec{w}= \pm \vec{w}^{*}, H^{-1}$ vanishes and the fibers of the map $Y \rightarrow \mathbb{R}^{3}$ collapse to points. Those points are the fixed points of $\mathrm{K}$. Away from those two points, the map $Y \rightarrow \mathbb{R}^{3}$ is a circle fibration and $\vec{a}$ is a connection on this fibration with curvature

$$
\vec{\nabla} \times \vec{a}=\nabla H .
$$

What we have just described is the Gibbons-Hawking form of the EguchiHansen metric.

The three Kahler forms on $Y$ are

$$
\vec{\omega}=(d \chi+\vec{a} \cdot d \vec{w}) \wedge d \vec{w}-\frac{1}{2} H d \vec{w} \times d \vec{w}
$$

where $(d \vec{w} \times d \vec{w})_{i}=\epsilon_{i j k} d w_{j} \wedge d w_{k}$. As a check, we note that with the action of $\mathrm{K}$ corresponding to the vector field $\partial / \partial \chi$, the moment map for 


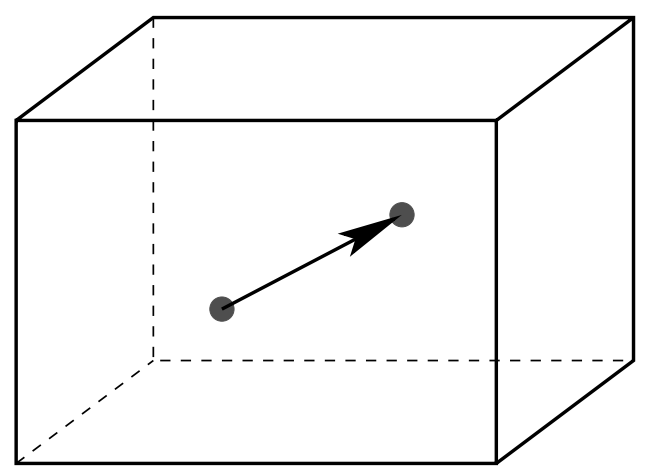

Figure 2: The space of $\vec{w} \in \mathbb{R}^{3}$. The tri-holomorphic action of $\mathrm{K} \simeq U(1)$ has fixed points at the two "Taub-NUT centers," $\vec{w}= \pm \vec{w}^{*}$, where $\vec{w}^{*}=$ $\frac{1}{2}(\alpha, \beta, \gamma)$. The inverse image in $Y$ of a straight line connecting these points is a two-sphere $S$.

$\mathrm{K}$ is indeed

$$
\vec{\mu}=\vec{w}
$$

as promised. A convenient choice of a two-surface $S$ that generates the second homology of $Y$ is (figure 2) the inverse image in $Y$ of a straight line in $\mathbb{R}^{3}$ from $\vec{w}=-\vec{w}^{*}$ to $\vec{w}=\vec{w}^{*}$. A short calculation shows that $\int_{S} \vec{w} / 2 \pi=2 \vec{w}^{*}$, justifying the formula (3.48) for the moduli.

Finally, we want to understand the action of $\tau$ and $\tilde{\tau}$. For $\tau$ or $\tilde{\tau}$ to be a symmetry, they must either (1) leave fixed the two points $\vec{w}= \pm \vec{w}^{*}$, which occurs if $\alpha=\gamma=0$, or (2) exchange them, which occurs if $\beta=0$.

$\tau$ and $\tilde{\tau}$ both commute with $\mathrm{K}$, and transform the symplectic forms by $\left(\omega_{I}, \omega_{J}, \omega_{K}\right) \rightarrow\left(-\omega_{I}, \omega_{J},-\omega_{K}\right)$. Since $\vec{w}$ is the moment map, this means that $\tau$ and $\tilde{\tau}$ acts on the base of $Y \rightarrow \mathbb{R}^{3}$ by $\left(w_{1}, w_{2}, w_{3}\right) \rightarrow\left(-w_{1}, w_{2},-w_{3}\right)$. Consequently, any $\mathrm{K}$-invariant $A$-brane that is pointwise $\tau$ or $\tilde{\tau}$-invariant must project under $\pi: Y \rightarrow \mathbb{R}^{3}$ to the $w_{2}$ axis, or a piece of it.

Given that $\tau$ and $\tilde{\tau}$ commute with rotations of the fiber and square to 1 , they can, roughly speaking, only act on $\chi$ by $\chi \rightarrow \chi$ or $\chi \rightarrow \chi+\pi$. Let us first discuss case $(2)$, with $\beta=0$. This is the case that is related to the principal series, and we know from Section 3.4 that there is a (pointwise) $\tilde{\tau}$-invariant $A$-brane with topology $\mathbb{R} \times S^{1}$. This brane must project to the full $w_{2}$ axis. Hence, $\tilde{\tau}$ must leave $\chi$ fixed in this case. On the other hand, for $\beta=0, \gamma \neq 0$, there is no such $\tau$-invariant $A$-brane, so $\tau$ must act by 
$\chi \rightarrow \chi+\pi$. Thus, for $\beta=0$, we have

$$
\begin{aligned}
& \tau: \chi \rightarrow \chi+\pi \\
& \tilde{\tau}: \chi \rightarrow \chi .
\end{aligned}
$$

On the other hand, consider the case $\alpha=\gamma=0, \beta>0$. The two fixed points are at $\left(w_{1}, w_{2}, w_{3}\right)= \pm \frac{1}{2}(0, \beta, 0)$. From Section 3.2, we know that there is an $S U(2)$-invariant brane with topology $S^{2}$ on which $\tau$ acts trivially. This brane must project to the part of the $w_{2}$ axis connecting the two fixed points. On the other hand, from Section 3.3, we know that there are two $\tilde{\tau}$-invariant branes with topology $\mathbb{R}^{2}$. These must correspond to the regions $w_{2} \geq \beta / 2$ and $w_{2} \leq-\beta / 2$ of the $w_{2}$ axis. The action on $\chi$ of $\tau$ and $\tilde{\tau}$ is therefore

$$
\begin{gathered}
\tau: \chi \rightarrow \begin{cases}\chi+\pi & \left|w_{2}\right|>\beta / 2 \\
\chi & \left|w_{2}\right|<\beta / 2\end{cases} \\
\tilde{\tau}: \chi \rightarrow \begin{cases}\chi & \left|w_{2}\right|>\beta / 2 \\
\chi+\pi & \left|w_{2}\right|<\beta / 2\end{cases}
\end{gathered}
$$

For $\beta \rightarrow 0,(3.53)$ and (3.52) coincide, as expected. In (3.53), we have not explained what happens for $\left|w_{2}\right|=\beta / 2$; these are the fixed point of $\mathrm{K}$ so the value of $\chi$ is immaterial there.

Now let us consider $Y$ as a symplectic manifold with symplectic form $\omega_{Y}=\omega_{K}=(d \chi+\vec{a} \cdot \vec{w}) \wedge d w_{3}-H d w_{1} \wedge d w_{2}$, and try to describe the Kinvariant Lagrangian submanifolds $M$. K-invariance means that $M$ is a union of fibers of the projection $Y \rightarrow \mathbb{R}^{3}$. So in fact, $M$ must be the inverse image in $Y$ of a one-dimensional curve $\ell$ in $\mathbb{R}^{3}$. The restriction of $\omega_{Y}$ to such an $M$ is the same as the restriction of $d \chi \wedge d w_{3}$, and vanishes if and only if $\ell$ is at a constant value of $w_{3}$. So to find $M$, we specify a constant value of $w_{3}$, which we call $b$, and we let $\mathbb{R}_{b}^{2} \subset \mathbb{R}^{3}$ be the plane defined by $w_{3}=b$. Then we pick any curve $\ell \subset \mathbb{R}_{b}^{2}$, and take $M$ to be its inverse image in $Y$.

The plane $\mathbb{R}_{b}^{2}$ may contain zero, one, or two of the special points $\vec{w}=$ $\pm \vec{w}^{*} . \mathbb{R}_{b}^{2}$ contains one special point if $\beta / 2= \pm b$, and both special points if $b=\beta=0$. In figure 3 , we sketch a case in which both of the special points are contained in $\mathbb{R}_{b}^{2}$; in this example, $\ell$ extends to infinity in both directions. Although a closed curve $\ell$ will lead to a Lagrangian submanifold, it does not generally lead to an $A$-brane. The reason is that a closed curve in $\mathbb{R}_{b}^{2}$ is the boundary of a disc instanton (holomorphic in complex structure $K$ ), so that such a Lagrangian submanifold is not likely to be the support of an 


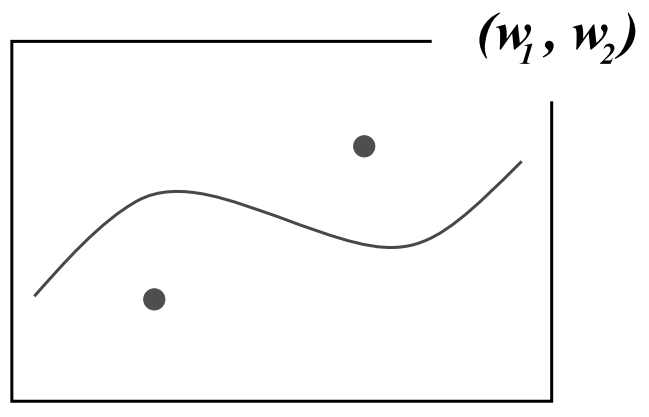

Figure 3: A K-invariant Lagrangian submanifold $M \subset Y$ is represented by a planar curve $\ell \in \mathbb{R}_{b}^{2}$. Plotted is the case that $\mathbb{R}_{b}^{2}$ contains both special points, depicted here as solid dots.

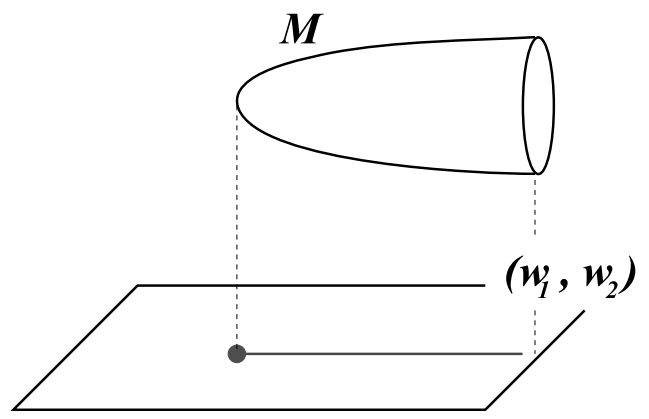

Figure 4: If $\ell$ is semi-infinite, running from a fixed point to infinity, then the corresponding Lagrangian submanifold is a semi-infinite cigar, equivalent topologically to $\mathbb{R}^{2}$.

$A$-brane. ${ }^{5}$ On the other hand, it is definitely possible for $\ell$ to terminate at one or both ends at one of the special points $\vec{w}= \pm \vec{w}^{*}$, where the fiber of the map $Y \rightarrow \mathbb{R}^{3}$ collapses to a point.

There are thus three topologies for $M$ :

(1) If $\ell$ connects the two points $\vec{w}= \pm \vec{w}^{*}$, the fiber collapses at both ends and $M$ is topologically a two-sphere.

(2) If $\ell$ is infinite at one end, and ends at the other end at one of the two special points, then the fiber collapses at one end and $M$ is topologically $\mathbb{R}^{2}$. (This is illustrated in figure 4 .)

(3) If $\ell$ is infinite at both ends, the fiber never collapses and $M$ is topologically $\mathbb{R} \times S^{1}$.

${ }^{5}$ Also, the space of $\left(\mathcal{B}_{\mathrm{cc}}, \mathcal{B}^{\prime}\right)$ strings cannot have an interpretation in terms of quantization if $\ell$ is a closed curve, because the condition for nondegeneracy of $\omega_{J}$ that we discuss shortly cannot be satisfied for a closed curve. 
We have already met $A$-branes of all three types. In Section 3.2, we associated finite-dimensional representations of $S U(2)$ with branes of type (1). In Section 3.3, we associated the discrete series with branes of type (2). And in Section 3.4, we associated the principal series with branes of type (3). All of these branes have their support on the $w_{2}$ axis, with $w_{1}=w_{3}=0$. Asymptotic $S L(2, \mathbb{R})$ invariance means that at infinity, if $\ell$ does get to infinity, $\ell$ must be sufficiently close to the $w_{2}$ axis. We do not know the right criterion for "sufficiently close," and our results will not depend on this very much. For simplicity, we will consider the case that $w_{1}$ is bounded at infinity.

For the space of $\left(\mathcal{B}_{\mathrm{cc}}, \mathcal{B}^{\prime}\right)$ strings to have an interpretation in terms of quantization, $\omega_{J}$ must be nondegenerate when restricted to $M$. Since $\omega_{J}=$ $(d \chi+\vec{a} \cdot \vec{w}) \wedge d w_{2}-H d w_{3} \wedge d w_{1}$, and $d w_{3}=0$ along $\ell$, this is equivalent to saying that $d w_{2}$ is everywhere nonzero along $\ell$. In other words, $w_{2}$ is everywhere a good coordinate along $\ell$, and $\ell$ can be described by an equation $w_{1}=f\left(w_{2}\right)$, for some function $f$. Hamiltonian transformations of $Y$ that commute with $\mathrm{K}$ can include, as a special case, area-preserving transformations of $\mathbb{R}_{b}^{2}$ that leave invariant the fixed points $\vec{w}= \pm \vec{w}^{*}$ (if either of these points lies in $\mathbb{R}_{b}^{2}$ ). Modulo such a transformation, the only invariants of $\ell$ are whether it ends at a fixed point and whether it goes to the left or right of such a point.

Let us consider an $\ell$ that is infinite at both ends and asymptotically parallel to the $w_{2}$ axis. Thus, the Lagrangian submanifold $M$ is topologically $\mathbb{R} \times S^{1}$. $\quad \ell$ may have complicated wiggles, but these are irrelevant in the $A$-model. To find the parameters in the $A$-model, we simply observe that as the first Betti number of $M$ is 1 , a rank $1 A$-brane supported on $M$ depends on a single complex parameter or a pair of real parameters. One real parameter is the Wilson line - the holonomy of the Chan-Paton line bundle around $S^{1}$. As in Section 3.4, we parametrize the holonomy of this bundle as $\exp (2 \pi \mathrm{i} \delta)$. Then $\delta$ is one real parameter characterizing a rank $1 A$-brane supported on $M$. The second real parameter corresponds to a displacement of $M$ by the vector field $V=\omega_{Y}^{-1} \zeta$, where $\zeta$ is a closed but not exact one-form on $M$, and $\omega_{Y}=\omega_{K}$ is the symplectic form of the $A$-model. In the case at hand, we can take $\zeta=d \chi+\vec{a} \cdot d \vec{w}$ (which is closed when restricted to $M$ ), in which case $V$ is the vector field $\partial / \partial w_{3}$, which shifts $b$. Thus, the second modulus is simply $b$. Moreover, the $A$-model is holomorphic in $\delta+i b$.

It is not hard to understand the meaning of the moduli. In quantizing $M$ with respect to symplectic structure $\omega_{J}$, the moment map for the $\mathrm{K}$ symmetry is $w_{2}$, which thus plays the role of $z$ in Sections 3.3-4. $w_{2}$ is unbounded below along $M$, since $\ell$ is asymptotically parallel to the $w_{2}$ 
axis. So the spectrum of $J_{z}$ eigenvalues in quantization of $M$ is of the form $\{s+n \mid n \in \mathbb{Z}\}$, for some complex number $s$ that is defined modulo 1. As in Section 3.4, the real part of $s$ is determined by the monodromy around $S^{1}$ of the Chan-Paton bundle of $M \cong \mathbb{R} \times S^{1}$. Thus Re $s=\delta+$ constant, so holomorphy of the $A$-model in $\delta+\mathrm{i} b$ implies that

$$
s=\delta+\mathrm{i} b+\text { constant }
$$

Now let us discuss what sort of branes are associated with modules that have a highest or lowest weight vector. For this, $\ell$ should end on one of the fixed points $\vec{w}^{*}= \pm \frac{1}{2}(\alpha, \beta, \gamma)$, and hence (taking the positive sign) we must have

$$
b=\gamma / 2 \text {. }
$$

According to (3.11),

$$
\mu=\beta+\mathrm{i} \gamma=\eta+\mathrm{i} \gamma+n
$$

with $n$ an integer. Comparing the last three formulas, the imaginary part of $\mu$ is twice the imaginary part of $s$, so via holomorphy, we have $\mu / 2=$ $s+n+$ constant. The constant is $1 / 2$, since, as we explained in Section 3.3, a highest or lowest weight module with $\mu=\beta=\gamma=0$ has $s=1 / 2$. So the quadratic Casimir operator is

$$
J^{2}=\left(\mu^{2}-1\right) / 4=(s+n+1 / 2)^{2}-1 / 4=(s+n)(s+n+1),
$$

which is the expected result for a module with a lowest or highest weight vector.

It is inevitable that there is an undetermined integer $n$ in this formula, since $s$ (which is defined by saying that the eigenvalues of $J_{z}$ are congruent to $s \bmod \mathbb{Z}$ ) is only determined modulo an additive integer. Moreover, the derivation involved no input that would distinguish a representation containing a highest weight vector from one containing a lowest weight vector. For a brane to have an interpretation in terms of quantization, $w_{2}$ must be a monotonic function along $\ell$, so either $\ell$ runs from $w_{2}=-\infty$ to the fixed point or it runs from the fixed point to $w_{2}=+\infty$. Since the value of $w_{2}$ is a classical approximation to $J_{z}$, the two types of brane correspond to highest weight and lowest weight representations, respectively. In either case, to determine the precise $J_{z}$ value of the highest or lowest weight vector, we must treat the quantization more precisely, or use an algebraic argument such as that explained in Section 3.5.

If one end of $\ell$ is at a fixed point (figure 4), the corresponding Lagrangian submanifold $M$ is topologically $\mathbb{R}^{2}$. The first Betti number of $M$ vanishes, so 
the corresponding brane $\mathcal{B}^{\prime}$ has no deformations as an $A$-brane. This is what we expect for a brane associated to a highest or lowest weight representation. The value of $s$ for such a brane is constrained to obey (3.57) for some integer $n$, so there is no possible deformation. ( $J^{2}$ is a property of the space $Y$, not the brane $\mathcal{B}^{\prime}$, so $J^{2}$ will remain fixed in deformations of $\mathcal{B}^{\prime}$ in the $A$-model of $Y$.)

Now let us consider a pair of curves $\ell_{1}$ and $\ell_{2}$, both ending at the fixed point. We suppose that $\ell_{1}$ runs from $-\infty$ to the fixed point and $\ell_{2}$ from the fixed point to $+\infty$. $\ell_{1}$ and $\ell_{2}$ correspond to $A$-branes $\mathcal{B}_{1}$ and $\mathcal{B}_{2}$ that are associated, respectively, to a highest weight representation $R_{1}$ and a lowest weight representation $R_{2}$ of $\mathfrak{s l}(2, \mathbb{R})$. $\mathcal{B}_{1}$ and $\mathcal{B}_{2}$ are supported on Lagrangian submanifolds $M_{1}$ and $M_{2}$, each of which is topologically $\mathbb{R}^{2}$. The two copies of $\mathbb{R}^{2}$ meet transversely. In terms of some local complex coordinates $u$ and $v$ (which we can take to be holomorphic in complex structure $J$ ), $M_{1}$ is defined by $v=0$ and $M_{2}$ by $u=0$.

Consider the reducible $A$-brane $\mathcal{B}^{\prime}=\mathcal{B}_{1} \oplus \mathcal{B}_{2}$, which is associated to the reducible representation $R=R_{1} \oplus R_{2}$, in which $J_{z}$ is bounded neither above nor below. It is supported on $M=M_{1} \cup M_{2}$, which is defined by the equation

$$
u v=0 .
$$

What are the deformations of $\mathcal{B}^{\prime}$ as an $A$-brane? The space of first-order deformations is the space of $\left(\mathcal{B}^{\prime}, \mathcal{B}^{\prime}\right)$ strings of ghost number 1 , which for $\mathcal{B}^{\prime}=\mathcal{B}_{1} \oplus \mathcal{B}_{2}$ decomposes as $\oplus_{i, j=1}^{2} \mathcal{H}_{i j}$, where $\mathcal{H}_{i j}$ is the space of $\left(\mathcal{B}_{i}, \mathcal{B}_{j}\right)$ strings of ghost number $1 . \mathcal{H}_{11}$ and $\mathcal{H}_{22}$ are each trivial, since the first Betti numbers of $M_{1}$ and $M_{2}$ are trivial. On the other hand, as $M_{1}$ and $M_{2}$ intersect transversely at a single point in $Y$, which is of codimension 4 , the spaces $\mathcal{H}_{12}$ and $\mathcal{H}_{21}$ are each of complex dimension 1. A general deformation of $\mathcal{B}^{\prime}$ is thus given by a pair of complex parameters $a \in \mathcal{H}_{12}$ and $b \in \mathcal{H}_{21}$.

If $a=b=0, \mathcal{B}^{\prime}$ remains as a direct sum. If $a \neq 0, b=0$, or $a=0, b \neq 0$, then $\mathcal{B}^{\prime}$ is deformed to an extension in one direction or the other, containing $\mathcal{B}_{1}$ or $\mathcal{B}_{2}$ as a sub-brane. This gives us three representations, with the same value of $s$, exactly in parallel with the situation that was described in Section 3.5 , for example in equations (3.40) and (3.41). In fact, the parameters $a$ and $b$ in the present derivation with branes correspond to $a_{n}$ and $b_{n}$ in the previous purely algebraic analysis.

Finally, if $a$ and $b$ are both nonzero, the support of the brane $\mathcal{B}^{\prime}$ is deformed from $u v=0$ to $u v=\epsilon$, where $\epsilon \sim a b$. The support of the deformed brane is topologically $\mathbb{R} \times S^{1}$, with first Betti number 1 . So it is possible 
to turn on a monodromy parameter $\delta$. Of the three real parameters $\delta$, $\operatorname{Re} \epsilon$, and $\operatorname{Im} \epsilon$, two (namely $\delta$ and, say, $\operatorname{Im} \epsilon$ ) represent a deformation in $s$ away from its initial value that obeyed equation (3.57). This deformation forces the brane and the associated representation to become irreducible. (Indeed, when $\epsilon \neq 0$, the brane no longer passes through the fixed point at $u=v=0$; when $\delta$ is varied, the different components are linked by a monodromy.) The third real parameter, say $\operatorname{Re} \epsilon$, represents a displacement of $\ell=\ell_{1} \cup \ell_{2}$ in the $w_{1}$ direction. This type of displacement is irrelevant in the topological $A$-model, though it is meaningful as a deformation in the underlying sigma-model of $Y$.

One can go one step farther and consider a situation in which $\ell$ terminates on a fixed point at each end; in other words, $\ell$ connects the two fixed points at $\vec{w}= \pm \vec{w}^{*}$. This leads to the same derivation with $\vec{w}^{*}$ replaced by $-\vec{w}^{*}$, and $s$ replaced by $-s$ in (3.57). Requiring that (3.57) should hold for both signs of $s$, we learn as usual that $2 s \in \mathbb{Z}$. The associated representations are finite-dimensional. We studied them from the viewpoint of $S U(2)$ in Section 3.2, and we will re-examine them in Section 3.8 from the viewpoint of $S L(2, \mathbb{R})$.

\section{$3.8 \tilde{\tau}$-Invariant branes with only asymptotic $S L(2, \mathbb{R})$ symmetry}

Here we will study a more general class of $\tilde{\tau}$-invariant $A$-branes associated to $\mathfrak{s l}(2, \mathbb{R})$ representations that admit a hermitian structure that may or may not be positive definite. Thus, we will re-examine in terms of branes questions that were considered algebraically at the end of Section 3.5.

We continue to describe $Y$ in a manifestly $S L(2, \mathbb{R})$-invariant way by the equation

$$
-x^{2}-y^{2}+z^{2}=\frac{\mu^{2}}{4} .
$$

In this description, $\tau$ and $\tilde{\tau}$ act by

$$
\begin{aligned}
& \tau:(x, y, z) \rightarrow(-\bar{x},-\bar{y}, \bar{z}) \\
& \tilde{\tau}:(x, y, z) \rightarrow(\bar{x}, \bar{y}, \bar{z}) .
\end{aligned}
$$

We begin with the case $\mu^{2}>0$. For the compact cycle $S$ that generates the second homology of $Y$, we can take the fixed point set of $\tau$. In the present description, this fixed point set is characterized by $(x, y, z)=(i \hat{x}, i \hat{y}, z)$, where $\hat{x}, \hat{y}$, and $z$ are real and $\hat{x}^{2}+\hat{y}^{2}+z^{2}=\mu^{2} / 4$. 
Let $\mathcal{B}^{\prime}$ be a rank one $A$-brane supported on $S$. Since $\tau$ acts trivially on $S$, the space $\mathcal{H}$ of $\left(\mathcal{B}_{\mathrm{cc}}, \mathcal{B}^{\prime}\right)$ strings gives a unitary representation of $S U(2)$, a fact that we exploited in Section 3.2.

Here we will look at the same brane from the point of view of $S L(2, \mathbb{C})$ and eventually $S L(2, \mathbb{R})$. First of all, the set $S$ is not $S L(2, \mathbb{C})$-invariant. Nevertheless, the $A$-brane $\mathcal{B}^{\prime}$ is $S L(2, \mathbb{C})$-invariant, simply because $S$ is compact. For $v \in \mathfrak{s l}(2, \mathbb{C})$, let $h_{v}$ be the Hamiltonian function that generates the vector field on $Y$ corresponding to $v$. Let $\hat{h}_{v}$ be any function on $Y$ of compact support that coincides with $h_{v}$ in a neighborhood of $S$. The action of $\hat{h}_{v}$ is trivial in the $A$-model (since Hamiltonian isotopies of compact support are trivial in the $A$-model) and this action on $\mathcal{B}^{\prime}$ coincides with the action of $h_{v}$. (We could make a similar argument concerning the action of an element of the group $S L(2, \mathbb{C})$ that is close to the identity.) This argument still goes through if $S$ is not compact but is asymptotically $S L(2, \mathbb{C})$-invariant. For example, we will presently consider a Lagrangian submanifold that is $S L(2, \mathbb{C})$-invariant on the complement of a compact set. This is certainly an adequate condition.

Since the brane $\mathcal{B}^{\prime}$ is $S L(2, \mathbb{C})$-invariant, the group $S L(2, \mathbb{C})$ must act on $\mathcal{H}$. Indeed, in any finite-dimensional representation of $S U(2)$, the representation matrices can be analytically continued to give an action of $S L(2, \mathbb{C})$. (By contrast, the group $S L(2, \mathbb{C})$ will generally not act in a Hilbert space that furnishes a representation of $S L(2, \mathbb{R})$, even a unitary one; such representations are generally associated to branes whose support is noncompact in an essential way, and the above argument does not go through.)

Though $S L(2, \mathbb{C})$ acts on the Hilbert space $\mathcal{H}$ obtained in quantization of $S$, it certainly does not preserve the positive-definite hermitian structure of $\mathcal{H}$ that arises from $\tau$ symmetry. In fact, $S L(2, \mathbb{C})$ does not preserve any hermitian structure on $\mathcal{H}$. However, $\mathcal{H}$ does admit an $S L(2, \mathbb{R})$-invariant hermitian structure (though not a positive one).

The reason for this is simply that $S$ is $\tilde{\tau}$-invariant, as well as $\tau$-invariant. $\tilde{\tau}$ acts on $S$ by

$$
(\hat{x}, \hat{y}, z) \rightarrow(-\hat{x},-\hat{y}, z)
$$

$\tilde{\tau}$ does not leave $S$ fixed pointwise (as $\tau$ does), but it does map $S$ to itself, and therefore maps $\mathcal{B}^{\prime}$ to itself. So the construction of Section 2.4 can be applied using $\tilde{\tau}$ symmetry to get a manifestly $S L(2, \mathbb{R})$-invariant hermitian structure on $\mathcal{H}$. 
By following the logic of equation (2.18), we can make this explicit. If $($, is the natural pairing between $\left(\mathcal{B}_{\mathrm{cc}}, \mathcal{B}^{\prime}\right)$ strings and $\left(\mathcal{B}^{\prime}, \mathcal{B}_{\mathrm{cc}}\right)$ strings, then the $S U(2)$-invariant hermitian pairing is $\left\langle\psi, \psi^{\prime}\right\rangle=\left(\Theta_{\tau} \psi, \psi^{\prime}\right)$ and the $S L(2, \mathbb{R})$ invariant pairing is $\left\langle\left\langle\psi, \psi^{\prime}\right\rangle\right\rangle=\left(\Theta_{\tilde{\tau}} \psi, \psi^{\prime}\right)$. Here $\Theta_{\tau}=\tau \Theta$ and $\Theta_{\tilde{\tau}}=\tilde{\tau} \Theta$. Finally, $\tilde{\tau}$ is the same as $h \tau$, where $h$ is the rotation in (3.61), which acts on the Lie algebra $\mathfrak{s l}(2, \mathbb{C})$ the same way that it does on the coordinates:

$$
h\left(J_{x}, J_{y}, J_{z}\right) h^{-1}=\left(-J_{x},-J_{y}, J_{z}\right) .
$$

So the relation between $\langle\langle\rangle$,$\rangle and \langle$,$\rangle is$

$$
\left\langle\left\langle\psi_{1}, \psi_{2}\right\rangle\right\rangle=\left\langle h \psi_{1}, \psi_{2}\right\rangle .
$$

Explicitly, we can now verify that $\langle\langle\rangle$,$\rangle is S L(2, \mathbb{R})$-invariant. Since $J_{x}, J_{y}$, and $J_{z}$ are hermitian with respect to $\langle\rangle,,(3.62)$ implies that $J_{z}$ is hermitian but $J_{x}$ and $J_{y}$ are antihermitian with respect to $\langle\langle\rangle$,$\rangle , which is$ the condition for $S L(2, \mathbb{R})$-invariance.

The fact that the same representation admits hermitian forms invariant under either $S U(2)$ or $S L(2, \mathbb{R})$ is related to the fact that these groups are "inner real forms" of $S L(2, \mathbb{C})$. This means the following. $S L(2, \mathbb{R})$ is the subgroup of $S L(2, \mathbb{C}$ ) characterized by $g=\bar{g}$ (where we regard $g \in S L(2, \mathbb{C}$ ) as a $2 \times 2$ complex unimodular matrix), while $S U(2)$ is characterized by $g=h \bar{g} h^{-1}$. An "outer form" (there are none for $S L(2, \mathbb{C})$ ) would be characterized by $g=\phi(\bar{g})$, where $g \rightarrow \phi(g)$ is an outer automorphism of order 2 .

Let us describe the hermitian form $\langle\langle\rangle$,$\rangle more explicitly in a basis of$ eigenstates of $J_{z}$. First consider a representation of odd dimension $n=$ $2 k+1$. We diagonalize $J_{z}$ with $J_{z} \psi_{s}=s \psi_{s}, s=-k,-k+1, \ldots, k$. Equation (3.62) determines $h$ up to multiplication by a constant $c$, which for the moment we will assume to be real:

$$
h \psi_{s}=c(-1)^{s} \psi_{s} .
$$

So the sign of $\left\langle\left\langle\psi_{s}, \psi_{s}\right\rangle\right\rangle$ is $c(-1)^{s}$. Thus the states $\psi_{s}$ have alternating positive and negative norms. We have actually seen this structure from an algebraic point of view in Section 3.5, in a problem (see equation (3.44)) that is closely related, as will become clear.

The value of $c$ depends upon how one lifts $\tilde{\tau}$ from an automorphism of $Y$ to an automorphism of the relevant Chan-Paton line bundles. When one has a hermitian form that is positive definite if the sign is chosen properly, that gives a natural choice. Otherwise, what is natural may depend upon the 
problem. One fairly natural way to pick a lift of $\tilde{\tau}$ is to pick a fixed point $p \in S$ of $\tilde{\tau}$, assuming that there is one (in the present case there are two fixed points $p_{ \pm}$defined by $\left.(\hat{x}, \hat{y}, z)=(0,0, \pm \mu / 2)\right)$, and require that $\tilde{\tau}$ acts trivially on the fiber at $p$ of the relevant Chan-Paton bundles. This gives a definite recipe for defining the hermitian form, but in general it depends on the choice of $p$.

A further subtlety arises for an even-dimensional representation of $S U(2)$. In this case, since $s$ is half-integral, in order to make $\langle\langle\psi, \psi\rangle\rangle$ real-valued, we need to pick $c$ imaginary (which will ensure that the eigenvalues of $h$ are real). Here it is fairly clear that there cannot be a preferred choice between $c=i$ and $c=-i$. Indeed, it can be shown that in the recipe mentioned in the last paragraph, the sign depends on the choice of fixed point $p_{ \pm}$.

\section{The complementary series}

We return to the framework of Section 3.7 and we consider an $A$-brane associated with a suitable curve $\ell$. However, here we will take $\ell$ to be simply the $w_{2}$ axis, $w_{1}=w_{3}=0$. For $\beta=0, \gamma \neq 0$, this gives the brane, studied in Section 3.4, whose quantization leads to the principal series. Here we will consider the opposite case $\alpha=\gamma=0, \beta>0$. We take $\mathcal{B}^{\prime}$ to be a rank 1 $A$-brane supported on $M=\pi^{-1}(\ell)$.

In this case, the two fixed points of $\mathrm{K}$ lie on $\ell$ at the points $\pm \frac{1}{2}(0, \beta, 0)$. Hence, geometrically one can divide $M$ into three pieces $M_{+} \cong \mathbb{R}^{2}, M_{0} \cong$ $S^{2}$, and $M_{-} \cong \mathbb{R}^{2}$, respectively, corresponding to $w_{2} \geq \beta / 2, \beta / 2 \geq w_{2} \geq$ $-\beta / 2$, and $w_{2} \leq-\beta / 2$. Corresponding to this, if the parameters are chosen correctly, $\mathcal{B}^{\prime}$ may be the direct sum of three $A$-branes $\mathcal{B}_{+}, \mathcal{B}_{0}$, and $\mathcal{B}_{-}$.

For this, we need $\eta=0$ and $\beta$ equal to an integer $n$, so that an $A$-brane with support $M_{0}$ exists. (Recall from Section 3.2 that the space of $\left(\mathcal{B}_{\mathrm{cc}}, \mathcal{B}_{0}\right)$ strings has dimension $\beta$.) The decomposition $\mathcal{B}^{\prime}=\mathcal{B}_{+} \oplus \mathcal{B}_{0} \oplus \mathcal{B}_{-}$further needs a condition on the Chan-Paton bundle of $\mathcal{B}^{\prime}$. The monodromy around the point of intersection of $M_{+}$and $M_{0}$ (or of $M_{0}$ and $M_{-}$) must be trivial, or else this monodromy will link the different components.

Though it is possible for $\mathcal{B}^{\prime}$ to have a decomposition as an $A$-brane, this is not the main situation that we wish to discuss. We will primarily be interested in parameters for which $\mathcal{B}^{\prime}$ is irreducible. Actually, for brevity we will focus on the case of $s=0$, leading to representations of $S L(2, \mathbb{R})$ in which the center acts trivially. One can achieve $s=0$ for any $\beta$ or $J^{2}$ by suitably adjusting the monodromy of the Chan-Paton bundle. If $s=$ 0 , then the representation of $S L(2, \mathbb{R})$ is irreducible unless $J^{2}=n(n+1)$ for some integer $n$. When this occurs, quantization of $M_{0}$ gives a Hilbert 
space of dimension $2 n+1$. This happens for $\beta=2 n+1$. (For $s=1 / 2$, the representation becomes reducible for $J^{2}=n(n+1)$ with $n \in \mathbb{Z}+1 / 2$; this happens for even $\beta$. We leave this case to the reader.)

Let us discuss the space $\mathcal{H}$ of $\left(\mathcal{B}_{\mathrm{cc}}, \mathcal{B}^{\prime}\right)$ strings. First of all, $M_{+}$and $M_{-}$are $S L(2, \mathbb{R})$-invariant, though $M_{0}$ is not. But $M_{0}$ is compact. So we are in a situation similar to the one we encountered above: since $M$ is $S L(2, \mathbb{R})$-invariant away from a compact set, $S L(2, \mathbb{R})$ acts naturally on $\mathcal{H}$. Furthermore, this action preserves a hermitian form (though not necessarily a positive-definite one) since $M$ is $\tilde{\tau}$-invariant. In fact, $\tilde{\tau}$ acts trivially on the components $M_{ \pm}$, while rotating the two-sphere $M_{0}$ by an angle $\pi$ around its points of intersection with $M_{ \pm}$. These statements have essentially been summarized in equation (3.53), according to which $\tilde{\tau}$ acts on the fiber of $\pi: Y \rightarrow \mathbb{R}^{3}$ by

$$
\tilde{\tau}: \chi \rightarrow \begin{cases}\chi & \left|w_{2}\right|>\beta / 2 \\ \chi+\pi & \left|w_{2}\right|<\beta / 2\end{cases}
$$

We consider first the case that $\beta$ is large, to get a useful semiclassical description. Also, we take $s=0$, so as to get a representation of $S L(2, \mathbb{R})$ with integer eigenvalues of $J_{z}$. All integers will appear, since the moment map of $J_{z}$, which is $w_{2}$, is unbounded above and below on $M$. So $\mathcal{H}$ has a basis $\psi_{n}$ with $J_{z} \psi_{n}=n \psi_{n}$. Semiclassically, $M_{+}, M_{0}$, and $M_{-}$support, respectively, states with $J_{z}$ (or its moment map $w_{2}$ ) greater than $\beta / 2$, between $\beta / 2$ and $-\beta / 2$, and less than $-\beta / 2$, respectively. Quantization of $M_{+}$or $M_{-}$gives a positive-definite hermitian form, since $\tilde{\tau}$ acts trivially, while quantization of $M_{0}$ gives an oscillatory quadratic form, as described above. So the norm of $\psi_{n}$ is positive for $|n|>\beta / 2$ and oscillates in sign for $|n|<\beta / 2$. Semiclassical reasoning justifies these statements except near $|n|=\beta / 2$, where the fact that $\mathcal{B}^{\prime}$ is actually irreducible becomes relevant. But actually, the algebraic analysis of the hermitian form in Section 3.5 shows that the statements are precisely valid.

Now let us consider the opposite region in which $\beta$ is small, still keeping $s=0$. At $\beta=0, M$ coincides with the brane used in Section 3.4 to describe the $\gamma=0$ case of the principal series. The hermitian form on $\mathcal{H}$ is certainly positive definite in that case; in fact, $\tilde{\tau}$ acts trivially on $M$ at $\beta=0$. When we turn on $\beta, J^{2}=\left(\beta^{2}-1\right) / 4$ becomes greater than $-1 / 4$. The $S L(2, \mathbb{R})$ action on $\mathcal{H}$ remains irreducible until we reach $\beta=1$. In this entire range, there is no highest or lowest weight vector (equations (3.35) and (3.36) show that for $s=0$ and $J^{2}<0$, there cannot be one), and the hermitian inner product remains positive definite, according to equation (3.44). What we have found is the complementary series of unitary representations of $S L(2, \mathbb{R})$. 
At $\beta=1$, the brane $\mathcal{B}^{\prime}$ is reducible. $\mathcal{H}$ splits up accordingly as $\mathcal{H}_{+} \oplus$ $\mathcal{H}_{0} \oplus \mathcal{H}_{-}$, where $\mathcal{H}_{ \pm}$are infinite-dimensional Hilbert spaces that realize the discrete series representations $D_{1}^{ \pm}$, and $\mathcal{H}_{0}$ is a one-dimensional trivial representation of $S L(2, \mathbb{R})$. The hermitian structure on $\mathcal{H}_{+} \oplus \mathcal{H}_{0} \oplus \mathcal{H}_{-}$ is not unique, as signs can be chosen independently on the three summands, depending on the lift of $\tilde{\tau}$ to act on the Chan-Paton bundles of the three branes. Continuing past $\beta=1$, the hermitian structure becomes unique again (up to a multiplicative constant, which we choose to get an almost positive-definite inner product), with a single state of negative norm. This follows from the algebraic analysis of Section 3.5 (see equation (3.44)). This result means that the hermitian structure at $\beta=1$ that continues smoothly to $\beta>1$ corresponds to taking the hermitian form on $\mathcal{H}_{+}, \mathcal{H}_{0}$, and $\mathcal{H}_{-}$to be respectively positive, negative, and positive.

If we continue to increase $\beta$, the brane $\mathcal{B}^{\prime}$ and the representation furnished by $\mathcal{H}$ are irreducible, as noted above, except when $\beta$ is an odd integer $2 n+1$, for some $n$. For $\beta$ between $2 n-1$ and $2 n+1$, the number of negative norm states is $n$, according to the algebraic analysis of Section 3.5. At $\beta=$ $2 n+1, \mathcal{H}$ decomposes as $\mathcal{H}_{+} \oplus \mathcal{H}_{0} \oplus \mathcal{H}_{-}$, where $\mathcal{H}_{ \pm}$furnish discrete series representations, and $\mathcal{H}_{0}$ is a representation of dimension $2 n+1$. Taking a basis of $\mathcal{H}_{0}$ with $J_{z} \psi_{k}=k \psi_{k},|k| \leq n$, the norm of $\psi_{k}$ has sign $c(-1)^{k}$, as explained earlier, where depending on the choice of lift of $\tilde{\tau}, c$ may be either 1 or -1 . One lift gives $n$ states of negative norm and one gives $n+1$. The lift that gives $n$ states of negative norm continues smoothly to $\beta<2 n+1$ and the one that gives $n+1$ states of negative norm continues smoothly to $\beta>2 n+1$. The fact that one lift extends in one direction and one in the other can be explained by a topological argument.

\section{$3.9 \quad$ Relation to $\mathcal{D}$-modules}

In Section 3.7, we showed how to describe a Harish-Chandra module in terms of a curve $\ell$ in the plane $\mathbb{R}_{b}^{2}$. We adopted as much as possible a topological point of view, not attempting to make a distinguished choice of $\ell$. Instead we required only that $w_{2}$ is a good coordinate along $\ell$, so that the space of $\left(\mathcal{B}_{\mathrm{cc}}, \mathcal{B}^{\prime}\right)$ strings in the $A$-model can be related to quantization of $M$.

However, certain choices of $\ell$ have particularly nice properties. One choice that is particularly nice from the point of view of quantization is to take $\ell$ to be a vertical line (or part of a vertical line) in the plane $\mathbb{R}_{b}^{2}$. In this case (figure 5), $M=\pi^{-1}(\ell)$ is a complex manifold in complex structure $J$. The associated $A$-brane $\mathcal{B}^{\prime}$ is then a brane of type $(A, B, A)$ (that is, it is a $B$-brane with respect to complex structure $J$ and an $A$-brane for any linear 


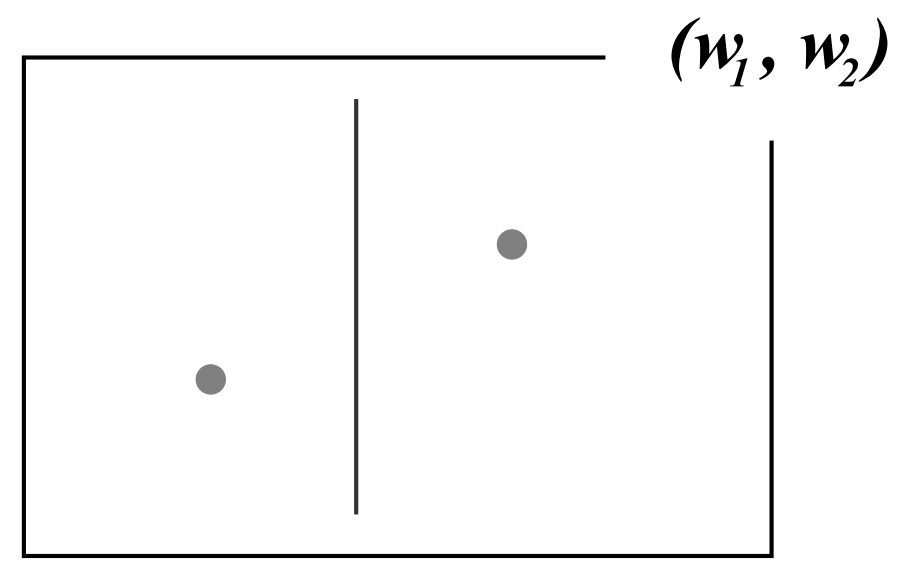

Figure 5: A vertical line in the plane $\mathbb{R}_{b}^{2}$, corresponding to a brane of type $(A, B, A)$.

combination of $\omega_{I}$ and $\left.\omega_{K}\right)$. This can be convenient, for the following reason. The canonical coisotropic brane $\mathcal{B}_{\mathrm{cc}}$ associated with a choice of hyper-Kahler metric on $Y$ is also a brane of type $(A, B, A)$. The space of $\left(\mathcal{B}_{\mathrm{cc}}, \mathcal{B}^{\prime}\right)$ strings in the $A$-model can be viewed as the space of string states of zero energy in the underlying sigma-model of $Y$ with $\mathcal{N}=4$ supersymmetry, or alternatively as the $\left(\mathcal{B}_{\mathrm{cc}}, \mathcal{B}^{\prime}\right)$ strings in the $B$-model of complex structure $J$. In other words, quantization in our sense coincides in this situation with what in geometric quantization is called quantization using the Kahler polarization determined by $J$. We exploited this relationship in Section 2.3 .

There is another type of choice for $\ell$ (figure 6) that is not closely related to quantization but is interesting from a different point of view. We take $\ell$ to run horizontally in $\mathbb{R}_{b}^{2}$, parallel to the $w_{1}$ axis. In this case, $M$ is holomorphic in complex structure $I$ and $\mathcal{B}^{\prime}$ is a brane of type $(B, A, A)$. This choice of $M$ is maximally unsuitable for an interpretation via quantization, because $\omega_{J}$ vanishes when restricted to $M$, rather than being nondegenerate. However, it has another virtue: it simplifies the relation between $A$-branes and $\mathcal{D}$-modules.

$Y$ can be interpreted as $T^{*} \mathbb{C P}^{1}$ if $\alpha \neq 0, \beta=\gamma=0$; more generally if $\beta$ and $\gamma$ are not zero, $Y$ is an affine deformation of $T^{*} \mathbb{C P}^{1}$. In any event, $Y$ admits an $S L\left(2, \mathbb{C}\right.$ )-invariant holomorphic map $\Psi: Y \rightarrow \mathbb{C P}^{1}$ (in fact two such maps, related in a sense by a Weyl transformation; explicit formulas are given below). In this situation, as explained in Section 11 of [6], the space of $\left(\mathcal{B}_{\mathrm{cc}}, \mathcal{B}^{\prime}\right)$ strings can be sheafified over $\mathbb{C P}^{1}$ and interpreted as the sheaf of sections of a twisted $\mathcal{D}$-module over $\mathbb{C P}^{1}$. The $\mathcal{D}$-module is twisted by $K^{1 / 2} \otimes \mathcal{O}(1)^{\lambda}$, where $K$ is the canonical line bundle of $\mathbb{C P}^{1}, \mathcal{O}(1)$ is the 


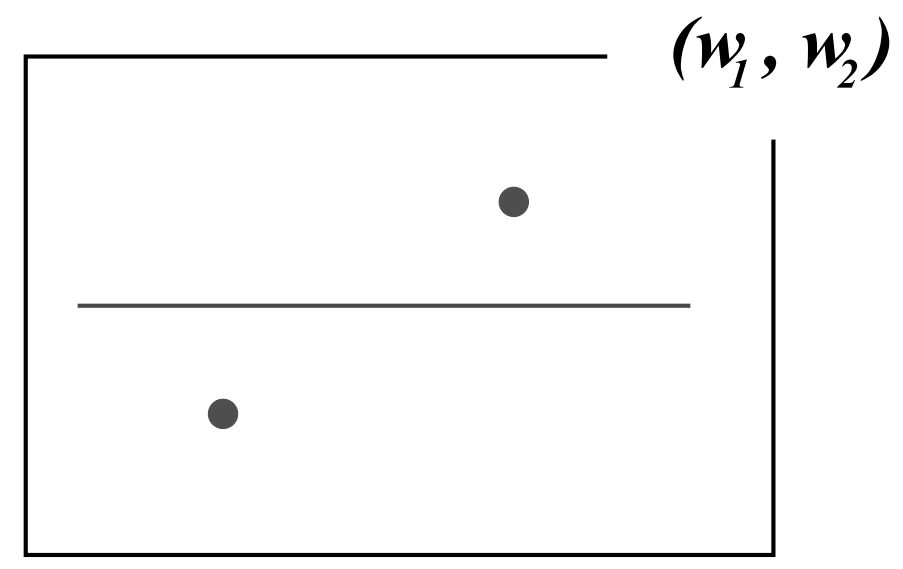

Figure 6: A horizontal line in the plane $\mathbb{R}_{b}^{2}$, corresponding to a brane of type $(B, A, A)$.

usual line bundle of degree 1 , and $\lambda=\eta+i \gamma$. (Such twisting is described in [28], Section 4.4.)

The fact that an $A$-brane leads to a twisted $\mathcal{D}$-module, as well as an $\mathfrak{s l}(2, \mathbb{C})$ module, enables us to make contact with the theory of Beilinson and Bernstein $[29]$ relating $\mathfrak{s l}(2, \mathbb{C})$ modules to twisted $\mathcal{D}$-modules on the flag manifold $\mathbb{C P}^{1}$. To compare to that theory, we would like to be able to explicitly describe the $\mathcal{D}$-module corresponding to a given $A$-brane. In general, this is difficult, but for branes of type $(B, A, A)$, there is a natural framework for doing so, as described in Section 4.3 of [24]. In general, this involves solving Hitchin's equations, but for the branes considered in the present paper, one can get an explicit answer as the relevant equations are abelian.

If $\mathcal{B}^{\prime}$ is a brane of type $(B, A, A)$ supported on $M \subset Y$ (so in particular $M$ is holomorphic in complex structure $I$ ), then the support of the corresponding $\mathcal{D}$-module is simply the projection of $M$ under the holomorphic map $\Psi: Y \rightarrow \mathbb{C P}^{1}$. In order to describe $\Psi$ explicitly, we recall that, in complex structure $I$, the complex symplectic manifold $Y$ is defined by the equation $x^{2}+y^{2}+z^{2}=\mu^{2} / 4$ in complex variables $x, y, z$. To an element of $Y$, we associated a complex $2 \times 2$ traceless matrix

$$
A=\left(\begin{array}{cc}
x & y-\mathrm{i} z \\
y+\mathrm{i} z & -x
\end{array}\right)
$$

with determinant $-\mu^{2} / 4$ or, equivalently, with eigenvalues $\pm \mu / 2$. Because $\mu / 2$ is an eigenvalue of $A$, there exists a nonzero column vector $\Upsilon$, unique 


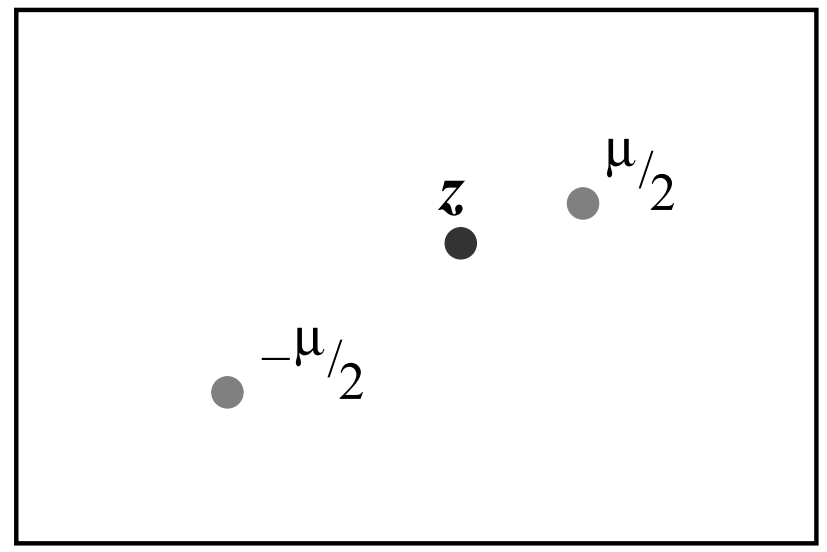

Figure 7: A brane $\mathcal{B}^{\prime}$ of type $(B, A, A)$ is located at a fixed value of $z=$ $w_{2}+\mathrm{i} w_{3}$.

up to scaling, that obeys

$$
A \Upsilon=\frac{\mu}{2} \Upsilon
$$

Up to scaling, $\Upsilon$ determines a point in $\mathbb{C P}^{1}$, and $\Psi$ is defined to map $A$ to this point. (A second such map can be defined by taking $\Upsilon$ to obey $A \Upsilon=-(\mu / 2) \Upsilon$.) Setting $\Upsilon=\left(\begin{array}{l}1 \\ t\end{array}\right)$ gives

$$
t=\frac{\mu / 2-z}{x-\mathrm{i} y}=\frac{x+\mathrm{i} y}{\mu / 2+z} .
$$

This formula explicitly describes the map $\Psi: Y \rightarrow \mathbb{C P}^{1}$. In terms of new variables $x_{ \pm}= \pm x+\mathrm{i} y$, we have

$$
t=\frac{z-\mu / 2}{x_{-}}=\frac{x_{+}}{\mu / 2+z} .
$$

In terms of these variables, the equation defining $Y$ becomes

$$
x_{+} x_{-}=z^{2}-\mu^{2} / 4 .
$$

The variable $z$ here can be identified with the complex variable $w_{2}+\mathrm{i} w_{3}$ in the Gibbons-Hawking description of the hyper-Kahler metric on $Y$.

Now let us return to branes of type $(B, A, A)$ (see figure 7 ). As we explained in Section 3.7, in the Gibbons-Hawking description of the hyperKahler metric on $Y$, a brane $\mathcal{B}^{\prime}$ of type $(B, A, A)$ is represented by a curve 
$\ell \subset \mathbb{R}^{3}$ with fixed values of $w_{2}$ and $w_{3}$. In other words, $\ell$ is a line parallel to the $w_{1}$ axis, or a part of it. In particular, since the value of $z=w_{2}+\mathrm{i} w_{3}$ is fixed, the support of this brane is a complex subvariety of $Y$ defined by a constant value of $x^{2}+y^{2}$ or equivalently of $x_{+} x_{-}$:

$$
x_{+} x_{-}=\epsilon .
$$

Here $\epsilon=-\mu^{2} / 4+z^{2}$ is a constant that vanishes precisely if $z= \pm \mu / 2$.

\section{Unbounded modules}

We start with the general case $\epsilon \neq 0$. In this case, $M$ is an irreducible algebraic curve defined by equation (3.71). It has the topology of $\mathbb{R} \times S^{1}$ and corresponds to a principal series representation of $S L(2, \mathbb{R})$ or its universal cover, or, more generally, to a Harish-Chandra module that has $J_{z}$ eigenvalues unbounded above and below. From (3.69) it follows that a brane $\mathcal{B}^{\prime}$ of this type corresponds to a $\mathcal{D}$-module supported on

$$
M^{\prime}=\mathbb{C P}^{1} \backslash\{0, \infty\} .
$$

Because the map from $M$ to $M^{\prime}$ is $1: 1$, the $\mathcal{D}$-module is of rank 1 , and is given by a flat bundle with structure group $\mathbb{C}^{*}=G L(1, \mathbb{C})$. As $\pi_{1}\left(M^{\prime}\right)=\mathbb{Z}$, such flat bundles are classified by a single element of $\mathbb{C}^{*}$, which we can take to be the monodromy around the origin (or the inverse of the monodromy at infinity). The logarithm of this monodromy corresponds to the parameter $s=\delta+\mathrm{i} b$ of Section 3.7. If the projection of $M$ to its image in $\mathbb{C P}^{1}$ were $k: 1$, we would have to solve rank $k$ Hitchin equations to describe the $\mathcal{D}$-module explicitly.

$M^{\prime}$ is one of the $\mathrm{K}_{\mathbb{C}}$ orbits in $\mathbb{C P}^{1}$. Indeed, the complex group $\mathrm{K}_{\mathbb{C}}=\mathbb{C}^{*}$ acts on the flag variety $\mathbb{C P}^{1}$, which is a union of three $\mathrm{K}_{\mathbb{C}}$ orbits: two compact orbits $\{0\}$ and $\{\infty\}$, and one open orbit $\mathbb{C}^{*} \simeq \mathbb{C P}^{1} \backslash\{0, \infty\}$.

\section{Highest and lowest weight modules}

As $z$ approaches one of the points $\pm \mu / 2$, say $\mu / 2$, we have $\epsilon \rightarrow 0$ in equation (3.71), and the brane $\mathcal{B}^{\prime}$ supported on the curve (3.71) degenerates into a sum of two branes, one supported at $x_{+}=0$, and the other supported at $x_{-}=0$. We denote these branes as $\mathcal{B}_{+}^{\prime}$ and $\mathcal{B}_{-}^{\prime}$, respectively. (The imaginary part of the condition $z=\mu / 2$ is $b=\gamma / 2$, which is precisely the condition (3.55) for $\ell$ to end at a special point.) From (3.69), it follows that the 
supports $M_{ \pm}^{\prime}$ of the branes $\mathcal{B}_{ \pm}^{\prime}$ are

$$
\begin{aligned}
& M_{+}^{\prime}=\{0\} \\
& M_{-}^{\prime}=\mathbb{C P}^{1} \backslash\{\infty\} .
\end{aligned}
$$

At the second special point, $z=-\mu / 2$, the roles of $\mathcal{B}_{+}^{\prime}$ and $\mathcal{B}_{-}^{\prime}$ are reversed and the branes $\mathcal{B}_{+}^{\prime}$ and $\mathcal{B}_{-}^{\prime}$ correspond to $\mathcal{D}$-modules supported on

$$
\begin{aligned}
M_{+}^{\prime} & =\mathbb{C P}^{1} \backslash\{0\} \\
M_{-}^{\prime} & =\{\infty\} .
\end{aligned}
$$

In both cases, the $\mathcal{D}$-modules supported on $M_{+}^{\prime}$ and $M_{-}^{\prime}$ correspond, respectively, to Harish-Chandra modules with lowest and highest weight vectors.

\subsection{Groups of higher rank}

We have concentrated on $S L(2, \mathbb{C})$ in this section to keep the arguments elementary, but there is a fairly immediate analog for any complex Lie group $G_{\mathbb{C}}$, with $Y$ taken to be a coadjoint orbit of $G_{\mathbb{C}}$, and $\Omega$ the natural holomorphic symplectic form of the coadjoint orbit. The algebra $\mathcal{A}$ of $\left(\mathcal{B}_{\mathrm{cc}}, \mathcal{B}_{\mathrm{cc}}\right)$ strings in that case is $\mathcal{U}\left(\mathfrak{g}_{\mathbb{C}}\right) / \mathcal{I}$, where $\mathcal{U}\left(\mathfrak{g}_{\mathbb{C}}\right)$ is the universal enveloping algebra of $G_{\mathbb{C}}$, and $\mathcal{I}$ is a deformation of the ideal that defines $Y$.

Suppose that $G_{\mathbb{C}}$ is of rank $r$. Then the ring of invariant polynomials on $\mathfrak{g}_{\mathbb{C}}$ is a polynomial ring with $r$ generators $\mathcal{O}_{1}, \ldots, \mathcal{O}_{r}$, which are known as Casimir operators. A regular orbit in $\mathfrak{g}_{\mathbb{C}}$ is obtained by setting the $\mathcal{O}_{i}$ to complex constants $c_{i}$. So if $Y$ is such a regular orbit, then the ideal $\mathcal{I}$ is generated by $\mathcal{O}_{i}-c_{i}^{\prime}$, with some constants $c_{i}^{\prime}, i=1, \ldots, r$ (which may differ from the classical values $c_{i}$, as we have seen for rank 1$)$.

For any Lagrangian $A$-brane $\mathcal{B}^{\prime}$, the space $\mathcal{H}$ of $\left(\mathcal{B}_{\mathrm{cc}}, \mathcal{B}^{\prime}\right)$ strings is a $\mathfrak{g}_{\mathbb{C}^{-}}$ module in which the values of the Casimir operators are $\mathcal{O}_{i}=c_{i}^{\prime}$. Thus, just as we saw for the rank 1 case, by making different choices of $\mathcal{B}^{\prime}$, we get many different $\mathfrak{g}_{\mathbb{C}}$-modules with the same values of the Casimir operators. Moreover, if $\mathcal{B}^{\prime}$ is invariant under an antiholomorphic involution of $Y$ (which leaves fixed the support of $\mathcal{B}^{\prime}$ ), then $\mathcal{H}$ furnishes a unitary representation of the corresponding real form of $G_{\mathbb{C}}$.

With suitable choices of $\mathcal{B}^{\prime}$, one can construct unitary representations of the compact form of $G_{\mathbb{C}}$ and analogs of the discrete and principal series for noncompact forms, as well as mixtures of these. The greatest novelty in going to rank greater than 1 is, however, that there are also interesting 
representations associated with nonregular orbits. For $S L(2, \mathbb{C})$, the only nonregular coadjoint orbit is the orbit of the zero element of $\mathfrak{s l}(2, \mathbb{C})$; this orbit is a point, leading to the trivial representation. Groups of higher rank have a greater variety of nonregular orbits, leading to nontrivial but in some sense small representations.

\section{Quantization of Chern-Simons gauge theory}

Finally, we conclude by examining one of the few known examples of a quantum field theory in which the subtleties of quantization actually play an important role.

The relevant quantum field theory is three-dimensional Chern-Simons gauge theory. It was analyzed in [30] and in more detail in $[15,31,32]$ from the viewpoint of geometric quantization, and has been studied from numerous other points of view ranging from conformal field theory to algebraic geometry and deformation quantization (references include [33], [34], and [35], respectively). See [36] for an introduction to the theory.

The aspect of interest to us is to construct the space of physical states of Chern-Simons theory with compact gauge group $G$ on an oriented twomanifold without boundary that we call $C$. For brevity, we take $G$ connected and simply connected. We define $M$ to be the moduli space of homomorphisms from $\pi_{1}(C)$ into $G$, up to conjugation, of a given topological type. $M$ (which is the classical phase space of the Chern-Simons theory) has a natural symplectic form $\omega_{*}$, inherited [37] from a symplectic structure on the infinite-dimensional linear space $\mathcal{A}$ of all connections on a $G$-bundle $E \rightarrow C$ of the appropriate topological type. In gauge theory, letting $A$ denote a connection on $E$, the symplectic form can be described explicitly by

$$
\omega_{*}=\frac{1}{4 \pi} \int_{C} \operatorname{Tr} \delta A \wedge \delta A .
$$

The symbol Tr represents an invariant quadratic form on the Lie algebra $\mathfrak{g}$ of $G$, which we normalize so that $\omega_{*} / 2 \pi$ is the image in de Rham cohomology of a generator of $H^{2}(M, \mathbb{Z}) \cong \mathbb{Z}$. (For $G=S U(n)$, Tr is the trace in the $n$-dimensional representation.) A line bundle $\mathcal{L}_{*}$ with curvature $\omega_{*}$ can be naturally constructed using gauge theory [38]. Then, picking a positive integer $\hat{k}$, we seek to quantize $M$ with symplectic form $\omega=\hat{k} \omega_{*}$ and prequantum line bundle $\mathcal{L}=\mathcal{L}_{*}^{\hat{k}}$.

To find a sigma-model that will do this, we need a natural complexification $Y$ of $M$, with certain properties. We simply take $Y$ to be the moduli space 
of homomorphisms from $\pi_{1}(C)$ to $G_{\mathbb{C}}$, the complexification of $G$, again up to conjugation. $Y$ is naturally a complex manifold. Indeed, $Y$ can be defined by giving the holonomies $U_{i}$ and $V_{j}, i, j=1, \ldots, g$ around a complete set of $a$-cycles and $b$-cycles in $C$. These are defined up to conjugation by an element of $G_{\mathbb{C}}$, and obey a single relation

$$
U_{1} V_{1} U_{1}^{-1} V_{1}^{-1} \ldots U_{g} V_{g} U_{g}^{-1} V_{g}^{-1}=1 .
$$

This description makes it clear that $Y$ is a complex manifold, and in fact an affine variety, with a great deal of holomorphic functions. We can describe these functions explicitly. Let $R$ be a finite-dimensional representation of $G_{\mathbb{C}}$. For $S$ an oriented closed loop on $C$, let $\operatorname{Hol}(S)$ be the holonomy of a flat connection around $S$. Then $W_{R}(S)=\operatorname{Tr}_{R} \operatorname{Hol}(S)$ is a holomorphic function on $Y$. (We took $R$ to be finite-dimensional to ensure that the trace always converges; for suitable infinite-dimensional $R$, the same formula gives a meromorphic function on $Y$.) In gauge theory, with $A$ now understood as a $\mathfrak{g}_{\mathbb{C} \text {-valued connection, we can write }}$

$$
W_{R}(S)=\operatorname{Tr}_{R} P \exp \left(-\oint_{S} A\right)
$$

Alternatively, if we write $S$ as a word in the $a$-cycles and $b$-cycles (regarded now as generators of $\pi_{1}(C)$ ), then $W_{R}(S)$ is the trace of the corresponding word in the $U_{i}$ and $V_{j}$. The holomorphic functions $W_{R}(S)$ restrict on $M$ to holonomy functions that we define and denote in the same way (the holonomies are now $G$-valued rather than $G_{\mathbb{C} \text {-valued). Thus the restrictions }}$ of holomorphic functions on $Y$ give a dense set of functions on $M$.

$Y$ has a nondegenerate holomorphic two-form $\Omega_{*}$ that is defined by the same formula as (4.1), with $A$ now understood as a $\mathfrak{g}_{\mathbb{C}}$ valued connection. We consider $Y$ as a complex symplectic manifold with holomorphic symplectic form $\Omega=\hat{k} \Omega_{*}$. Clearly, the restriction of $\Omega$ to $M$ coincides with $\omega$. The line bundle $\mathcal{L} \rightarrow M$ extends to a unitary line bundle $\mathcal{L} \rightarrow Y$ with a connection of curvature $\operatorname{Re} \Omega$.

As usual, we want to consider the $A$-model of $Y$ with symplectic structure $\omega_{Y}=\operatorname{Im} \Omega$. (This is the same $A$-model that is studied in the gauge theory approach to geometric Langlands [6], though the motivation there is a little different.) $Y$ is a space with a very good $A$-model, since in fact [39] it can be endowed with a complete hyper-Kahler metric extending its structure as a complex symplectic manifold. To find such a hyper-Kahler metric, one picks a complex structure on $C$, which enables one to write down Hitchin's equations; one then endows $Y$ with a complete hyper-Kahler metric by interpreting it as the moduli space of solutions of those equations. An important 
point here is that the structure of $Y$ as a complex symplectic manifold is completely natural (requiring no structure on $C$ except an orientation). But to endow $Y$ with a hyper-Kahler structure, which is useful for making the $A$-model concrete, we have to pick a complex structure on $C$. The choice of such a hyper-Kahler metric is a hyper-Kahler polarization of $(Y, M)$ in a sense described in Sections 1.3 and 2.3.

$G_{\mathbb{C}}$ has an antiholomorphic involution that keeps $G$ fixed; we write it as $U \rightarrow \bar{U}$ and call it complex conjugation. We define an antiholomorphic involution $\tau: Y \rightarrow Y$ that acts by complex conjugating all monodromies. $M$ is a component of the fixed point set of $\tau$, since by definition, $M$ is the locus in $Y$ with $G$-valued monodromies. (The fixed point set of $\tau$ has other components, as explained in [39].)

To place quantization of $M$ in the framework of this paper, we must as usual introduce two branes in the $A$-model of $Y$. One brane is the canonical isotropic brane $\mathcal{B}_{\mathrm{cc}}$, whose support is all of $Y$ and whose curvature form is $\operatorname{Re} \Omega$. This brane exists and is unique up to isomorphism because we have taken $G$ to be simply connected and $\hat{k}$ to be an integer. Restricted to $M$, $\operatorname{Re} \Omega$ is the symplectic form $\omega=\hat{k} \omega_{*}$ of $M$, which we wish to quantize. We let $\mathcal{B}^{\prime}$ be a rank $1 A$-brane supported on $M$; it exists and is unique up to isomorphism as $M$ is a simply connected spin manifold.

The space $\mathcal{H}$ of $\left(\mathcal{B}_{\mathrm{cc}}, \mathcal{B}^{\prime}\right)$ strings in the $A$-model gives a quantization of $M$ with symplectic structure $\omega$. Diffeomorphisms of $C$ that are continuously connected to the identity act trivially on $Y$ and on its $A$-model. (They do not preserve a hyper-Kahler metric on $Y$ that we may use to facilitate computations in the $A$-model, but $A$-model observables do not depend on this hyper-Kahler metric.) However, the mapping class group $\mathcal{M}_{C}$ of $C$ acts on $Y$ in a way that is nontrivial (and not isotopic to the identity) so it can act nontrivially on $\mathcal{H}$. It is useful to introduce the Teichmuller space $\mathcal{T}$ of $C$. Any point $t \in \mathcal{T}$ determines a complex structure on $C$ (unique up to isotopy) and hence a hyper-Kahler polarization of $(Y, M)$. We denote as $\mathcal{H}_{t}$ the space of $\left(\mathcal{B}_{\mathrm{cc}}, \mathcal{B}^{\prime}\right)$ strings constructed with this polarization. It is locally independent of $t$, since the $A$-model is invariant under a local change in the hyper-Kahler polarization, so the $\mathcal{H}_{t}$ fit together as fibers of a flat vector bundle over $\mathcal{T}$. Taking the monodromy of the flat connection, we get an action of $\mathcal{M}_{C}$ on $\mathcal{H}_{t}$ (for any choice of $t$ ). Actually, to be more precise, as is known from other approaches cited at the beginning of this section, what acts on $\mathcal{H}$ is a central extension of $\mathcal{M}_{C}$. Though the occurrence of a central extension is not surprising in quantum mechanics, to compute the central extension from the present point of view, we would need a better understanding of how to explicitly construct the flat connection. 
As explained in general in Section 2.3, after a choice of hyper-Kahler polarization corresponding to a point $t \in \mathcal{T}, \mathcal{H}$ can be computed explicitly as a vector space by taking the space of holomorphic sections of the appropriate line bundle,

$$
\mathcal{H}=H^{0}\left(M, \mathcal{L}_{*}^{\hat{k}} \otimes K^{1 / 2}\right),
$$

but the proper Hilbert space structure of the $A$-model is not given by an elementary formula in terms of the Kahler geometry of $M$. That is actually the standard result in Chern-Simons gauge theory; $\mathcal{H}$ can be constructed as a vector space by taking a suitable space of holomorphic sections, but that does not lead to a simple expression for the Hilbert space structure. Equation (4.4) can be slightly simplified using the fact that $K^{1 / 2} \cong \mathcal{L}_{*}^{-h}$, where $h$ is the dual Coxeter number of $G$. (This fact can be proved using the index theorem for a family of Dirac operators.) The standard algebrogeometric description of the physical Hilbert space of Chern-Simons theory at level $k$ is $\mathcal{H}=H^{0}\left(M, \mathcal{L}_{*}^{k}\right)$. So $k$ as conventionally defined in gauge theory or two-dimensional current algebra is related to $\hat{k}$ in the $A$-model by

$$
k=\hat{k}-h, \quad \hat{k}=k+h .
$$

Many formulas in Chern-Simons gauge theory are most simply written not in terms of the underlying coupling $k$ but in terms of $k+h$, which, as we now see, is the natural $A$-model parameter $\hat{k}$.

The holonomy functions $W_{R}(S)$ generate, classically, a commutative algebra. In the $A$-model, this commutative algebra is deformed as usual to a noncommutative algebra $\mathcal{A}$, the space of $\left(\mathcal{B}_{\mathrm{cc}}, \mathcal{B}_{\mathrm{cc}}\right)$ strings. This algebra will act on $\mathcal{H}$. In Chern-Simons gauge theory, what this means is simply that Wilson loops on $C$, upon quantization, become operators that act on the quantum Hilbert space. Some aspects of this were described in [30]; for more from the point of view of deformation quantization, and additional references, see [35].

The greatest novelty of the present approach to this much-studied subject is probably that it is clear that the very same algebra $\mathcal{A}$ acts on the space of $\left(\mathcal{B}_{\mathrm{cc}}, \mathcal{B}\right)$ strings for any other choice of $A$-brane $\mathcal{B}$. Quite a few interesting choices can be contemplated. We conclude by mentioning some illustrative examples.

We have already considered one case, in which $\mathcal{B}$ is a rank $1 A$-brane supported on $M$, and the space $\mathcal{H}$ of $\left(\mathcal{B}_{\mathrm{cc}}, \mathcal{B}\right)$ strings can be interpreted as a quantization of $M$. 
Alternatively, since the $A$-model we are considering here is the same one that is considered in the gauge theory approach to geometric Langlands, we can consider $A$-branes that are important in that context. These are rank 1 $A$-branes supported on a fiber of the Hitchin fibration. The Hitchin fibration is a map $\pi: Y \rightarrow \boldsymbol{B}$, where $\boldsymbol{B}$ is an affine space of half the dimension of $Y$. The map is holomorphic not in the natural complex structure of $Y$ with which we began the discussion but in another complex structure ${ }^{6}$ that is found by solving Hitchin's equations. A generic fiber $\boldsymbol{F}$ of the Hitchin fibration is a torus that is a complex abelian variety from the point of view of this other complex structure. More relevant for our present purposes, $\boldsymbol{F}$ is a Lagrangian submanifold from the point of view of $\omega_{Y}=\operatorname{Im} \Omega$, so it can be the support of a rank $1 A$-brane $\mathcal{B}^{*}$. Moreover, Re $\Omega$ is nondegenerate when restricted to $\boldsymbol{F}$, so the space $\mathcal{H}^{*}$ of $\left(\mathcal{B}_{\mathrm{cc}}, \mathcal{B}^{*}\right)$ strings can be regarded as a quantization of $\boldsymbol{F}$.

Since $\boldsymbol{F}$ is a torus, one would naively expect quantization of $\boldsymbol{F}$ to be related to abelian current algebra, not nonabelian current algebra. However, there should be a close relation between $\mathcal{H}$ and $\mathcal{H}^{*}$, because a certain very singular special fiber of the Hitchin fibration (the fiber at the "origin") has $M$, taken with multiplicity greater than 1 , as one of its components. This fact should lead to an embedding of $\mathcal{H}$ (or possibly of the direct sum of several copies of $\mathcal{H}$ ) in $\mathcal{H}^{*}$, something that is very likely related to various results in conformal field theory in which current algebra of the nonabelian group $G$ is expressed in terms of an abelian current algebra.

For a quite different kind of example, let $G_{\mathbb{R}}$ be an arbitrary real form of the complex Lie group $G_{\mathbb{C}}$. For any such real form, there is an antiholomorphic involution $\phi: G_{\mathbb{C}} \rightarrow G_{\mathbb{C}}$ that leaves $G_{\mathbb{R}}$ fixed. Mapping the holonomies $U_{i}, V_{j}$ to their transforms by $\phi$ gives an antiholomorphic involution of $Y$ that we call $\tau_{\phi}$. The fixed point set of $\tau_{\phi}$ has a component that is the moduli space $M_{\phi}$ of $G_{\mathbb{R}^{-}}$valued flat connections on $C$. If $G_{\mathbb{R}}$ is compact, then $M_{\phi}$ simply coincides with the space $M$ whose quantization we have already discussed.

In general, $M_{\phi}$ is the phase space of three-dimensional Chern-Simons theory with gauge group $G_{\mathbb{R}}$, compactified on a two-manifold $C$. It is a Lagrangian submanifold of $Y$ with respect to $\omega_{Y}=\operatorname{Im} \Omega$ and supports a rank $1 A$-brane $\tilde{\mathcal{B}}$. The space $\tilde{\mathcal{H}}$ of $\left(\mathcal{B}_{\mathrm{cc}}, \tilde{\mathcal{B}}\right)$ strings can be interpreted as the space of physical states in quantization on $C$ of Chern-Simons theory with gauge group $G_{\mathbb{R}}$. One interesting consequence of the present point of view is

\footnotetext{
${ }^{6}$ This complex structure is called $I$ in [39]. There is a difficult-to-avoid clash in notation with Section 2, where the analogous complex structure was called $J$. The notation there was motivated by compatibility with the gauge theory approach to geometric Langlands.
} 
that the same algebra $\mathcal{A}$ of quantized holonomies that acts in the compact case also acts on the space of physical states for any noncompact real form. This is almost clear perturbatively (except for a subtlety at the one-loop level [40], which we explain shortly), but is less obvious nonperturbatively.

To describe $\tilde{\mathcal{H}}$ explicitly, we pick again a point $t \in \mathcal{T}$, giving a hyperKahler polarization of $\left(Y, M_{\phi}\right)$. In other words, after picking $t$, we solve Hitchin's equations to get a hyper-Kahler metric on $Y$, which determines a complex structure on $M_{\phi}$. Then in this hyper-Kahler polarization, $\tilde{\mathcal{H}}_{t}$ is explicitly $H^{0}\left(M_{\phi}, \mathcal{L}_{*}^{\hat{k}} \otimes K^{1 / 2}\right)$, where now $K$ is the canonical bundle of $M_{\phi}$. However, it is not true that $K^{1 / 2} \cong \mathcal{L}_{*}^{-h}$. Rather, $K^{1 / 2} \cong \mathcal{L}_{*}^{-h_{\phi}}$, where $h_{\phi}$ is defined as follows. Decompose the Lie algebra of $G_{\mathbb{R}}$ as $\mathfrak{g}=\mathbf{k} \oplus \mathfrak{p}$, where $\mathbf{k}$ is the Lie algebra of a maximal compact subgroup of $G_{\mathbb{R}}$ and $\mathfrak{p}$ is its orthocomplement. After expressing $h$ in terms of the trace in $\mathfrak{g}$ of the square of a suitable element of $\mathfrak{g}$, write $h=h_{+}+h_{-}$, where $h_{+}$and $h_{-}$come from traces in $\mathbf{k}$ and $\mathfrak{p}$, respectively. Then (as one can again prove using the families index theorem) $h_{\phi}=h_{+}-h_{-}$. We thus have

$$
\tilde{\mathcal{H}}_{t}=H^{0}\left(M_{\phi}, \mathcal{L}_{*}^{\hat{k}-h_{\phi}}\right) .
$$

We would like to compare this result to Chern-Simons gauge theory, but it is difficult to do so because Chern-Simons gauge theory of a noncompact gauge group is not well-understood. However, it is a reasonable conjecture that the space of physical states is $\tilde{\mathcal{H}}_{t}=H^{0}\left(M_{\phi}, \mathcal{L}_{*}^{k_{\phi}}\right)$, where $k_{\phi}$ is the Chern-Simons coupling. If so, the relation between $k_{\phi}$ and the $A$-model parameter $\hat{k}$ is

$$
k_{\phi}=\hat{k}-h_{\phi}, \quad \hat{k}=k_{\phi}+h_{\phi} .
$$

Equivalently, for the same algebra $\mathcal{A}$ to act in Chern-Simons theory with compact gauge group and coupling $k$ as in Chern-Simons gauge theory with gauge group $G_{\mathbb{R}}$ and coupling $k_{\phi}$, the relation between $k$ and $k_{\phi}$ must be

$$
k+h=k_{\phi}+h_{\phi} .
$$

The couplings are here defined so that the spaces of physical states are $H^{0}\left(M, \mathcal{L}_{*}^{k}\right)$ and $H^{0}\left(M_{\phi}, \mathcal{L}_{*}^{k_{\phi}}\right)$, respectively. These formulas are consistent with a computation [40] of the one-loop quantum correction in ChernSimons theory with a noncompact gauge group.

Either the topological invariance of Chern-Simons gauge theory or the fact that the $A$-model is independent of a choice of hyper-Kahler polarization 
implies that there should be a natural projectively flat connection governing the dependence of $\tilde{\mathcal{H}}_{t}$ on $t$. Except in the compact case, it is not known how to explicitly construct such a connection. However, for $G_{\mathbb{R}}$ a split real form, and a particular component of $M$ (the one that is contractible topologically), the appropriate representation of the mapping class group has been constructed by another method based on real polarizations [41].

\section{Acknowledgments}

We would like to thank D. Kazhdan, M. Kontsevich, N. Hitchin, and P. Sarnak for valuable discussions. Research of SG is supported in part by NSF Grants DMS-0635607 and PHY-0757647, in part by RFBR grant 0702-00645, and in part by the Alfred P. Sloan Foundation. Research of EW is supported in part by NSF Grant PHY-0503584. Conclusions reported here are those of the authors and not of funding agencies.

\section{References}

[1] B. Kostant, Line bundles and the prequantized Schrodinger equation, Coll. Group Theoret. Methods Phys. 4 (1972) 81.

[2] J.-M. Souriau, Quantification geometrique, Comm. Math. Phys. 1 (1966), 374.

[3] M. Kontsevich, Deformation quantization of algebraic varieties, Lett. Math. Phys. 56 (2001), 271-294 [arXiv:math/0106006].

[4] G. Tian and S.-T. Yau, Complete Kahler manifolds with zero Ricci curvature, I J. Amer. Math. Soc. 3 (1990), 579-609.

[5] A. Kapustin and D. Orlov, Remarks on A-branes, mirror symmetry, and the Fukaya category, J. Geom. Phys. 48 (2003), 84 [arXiv:hep-th/0109098].

[6] A. Kapustin and E. Witten, Electric-magnetic duality and the geometric langlands program, Comm. Number Theory Phys. 1 (2007), 1-236 [arXiv:hep-th/0604151].

[7] P. Bressler and Y. Soibelman, Mirror symmetry and deformation quantization [arXiv:hep-th/0202128].

[8] A. Kapustin, A-branes and noncommutative geometry [arXiv:hep-th/0502212].

[9] V. Pestun, Topological strings in generalized complex space, [arXiv:hep-th/0512189].

[10] M. Gualtieri, Branes on poisson varieties [arXiv:0710.2719]. 
[11] N. Hitchin, Generalized Calabi-Yau manifolds [arXiv:math/0209099].

[12] M. Gualtieri, Generalized complex geometry [arXiv:math/0401221].

[13] D. Nadler and E. Zaslow, Constructible sheaves and the Fukaya category [arXiv:math/0604379].

[14] M. Aldi and E. Zaslow, Coisotropic branes, noncommutativity, and the mirror correspondence, JHEP 0506 (2005), 019 [arXiv:hep-th/0501247].

[15] S. Axelrod, S. Della Pietra and E. Witten, Geometric quantization of Chern-Simons gauge theory, J. Diff. Geom. 33 (1991), 787.

[16] F. Bayen, M. Flato, C. Fronsdal, A. Lichnerowicz and D. Sternheimer, Deformation theory and quantization. 1. Deformations of symplectic structures, Ann. Phys. 111 (1978), 61.

[17] M. Kontsevich, Deformation quantization of Poisson manifolds, Lett. Math. Phys. 66 (2003), 157-216 [arXiv:q-alg/9709040].

[18] A. S. Cattaneo and G. Felder, A path integral approach to the Kontsevich quantization formula, Commun. Math. Phys. 212 (2000), 591 [arXiv:math/9902090].

[19] A. Connes, M. R. Douglas and A. S. Schwarz, Noncommutative geometry and matrix theory: compactification on tori, JHEP 9802 (1998), 003 [arXiv:hep-th/9711162].

[20] R. Brylinski, Geometric quantization of real minimal nilpotent orbits, Diff. Geom. Appl. 9 (1998), 5.

[21] R. Brylinski, Quantization of the 4-dimensional nilpotent orbit of SL(3, R), Canad. J. Math. 49 (1997), 916.

[22] R. Brylinski, Instantons and Kaehler geometry of nilpotent orbits, in "Representation Theories and Algebraic Geometry," ed. A. Broer, NATO Adv. Sci. Inst. Ser. C Math. Phys. Sci., 514, Kluwer Acad. Publ., Dordrecht, 1998, 85.

[23] A. Belov-Kanel, M. Kontsevich, Automorphisms of the Weyl algebra, Lett. Math. Phys. 74 (2005), 181-199 [arXiv:math/0512169].

[24] D. Freed and E. Witten, Anomalies in string theory with D-branes, Asian J. Math. 3 (1999), 819-52 [arXiv:hep-th/9907189].

[25] A. Chervov, L. Rybnikov, Deformation quantization of submanifolds and reductions via Duflo-Kirillov-Kontsevich map, EJTP 4, (15) (2007), 71-90 [arXiv:hep-th/0409005].

[26] I. M. Gelfand, M. I. Graev and I. I. Pyatetskii-Shapiro, Representation theory and automorphic functions, Academic Press, 1990.

[27] B. Kostant, On Laguerre polynomials, Bessel functions, Hankel transform and a series in the unitary dual of the simply-connected covering group of $S L(2, \boldsymbol{R})$, Represent. Theory 4 (2000), 181-224. 
[28] S. Gukov and E. Witten, Gauge theory, ramification, and the geometric langlands program [arXiv:hep-th/0612073].

[29] A. Beilinson and J. Bernstein, Localisation de $\mathfrak{g}$-Modules, C. R. Acad. Sci. Paris Ser. I Math. 292 (1981), 15-18.

[30] E. Witten, Quantum field theory and the Jones polynomial, Commun. Math. Phys. 121 (1989), 351.

[31] S. Elitzur, G. W. Moore, A. Schwimmer and N. Seiberg, Remarks on the canonical quantization of the Chern-Simons-Witten theory, Nucl. Phys. B 326 (1989), 108.

[32] N. Hitchin, Flat connections and geometric quantization, Comm. Math. Phys. 131 (1990), 347-380.

[33] A. Tsuchiya, K. Ueno, and Y. Yamada, Conformal field theory on universal family of stable curves with gauge symmetries, in 'Integrable Systems In Quantum Field Theory And Statistical Mechanics', Adv. Stud. Pure Math., 19, Academic Press, Boston, 1989, 495-566.

[34] G. Faltings, Stable G-bundles and projective connections, J. Alg. Geom. 2 (1993), 507-68.

[35] J. E. Anderson, Hitchin's connection, Toeplitz operators and symmetry invariant deformation quantization [arXiv:math/0611126].

[36] M. F. Atiyah, The geometry and physics of knots, Cambridge University Press, Cambridge, 1990.

[37] M. F. Atiyah and R. Bott, Yang-Mills equations over Riemann surfaces, Phil. Trans. R. Soc. Lond. A308 (1983), 523-615.

[38] T. R. Ramadas, I. M. Singer and J. Weitsman, Some comments on Chern-Simons gauge theory, Commun. Math. Phys. 126 (1989), 409.

[39] N. Hitchin, The self-duality equations on a Riemann surface, Proc. London Math. Soc. (3) 55 (1987), 59-126.

[40] D. Bar-Natan and E. Witten, Perturbative expansion of Chern-Simons gauge theory with non-compact gauge group, Commun. Math. Phys. 141 (1991), 423-440.

[41] V. V. Fock and A. Goncharov, Dual Teichmuller and lamination spaces [arXiv:math/05120312]. 